Nevada

Environmental

Restoration

Project

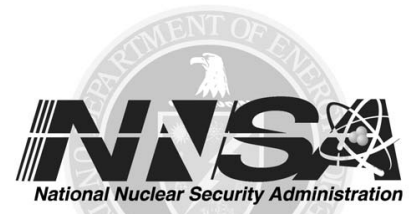

Post-Closure Monitoring Report for Corrective Action Unit 329:

Area 22 Desert Rock Airstrip Fuel Spill, Nevada Test Site, Nevada

Controlled Copy No.:

Revision No.: 0

June 2002

Approved for public release; further dissemination unlimited.

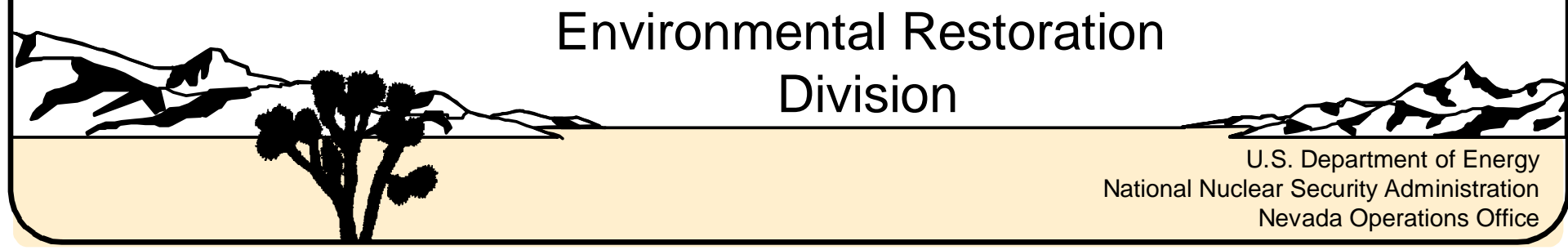


Available for public sale, in paper, from:

U.S. Department of Commerce

National Technical Information Service

5285 Port Royal Road

Springfield, VA 22161

Phone: 800.553 .6847

Fax: 703.605.6900

Email: orders@ntis.fedworld.gov

Online ordering: http://www.ntis.gov/ordering.htm

Available electronically at http://www.doe.gov/bridge

Available for a processing fee to U.S. Department of Energy and its contractors, in paper, from:

U.S. Department of Energy

Office of Scientific and Technical Information

P.O. Box 62

Oak Ridge, TN 37831-0062

Phone: 865.576 .8401

Fax: 865.576.5728

Email: reports@adonis.osti.gov

Reference herein to any specific commercial product, process, or service by trade name, trademark, manufacturer, or otherwise, does not necessarily constitute or imply its endorsement, recommendation, or favoring by the United States Government or any agency thereof or its contractors or subcontractors. 


\title{
POST-CLOSURE MONITORING REPORT FOR CORRECTIVE ACTION UNIT 329: AREA 22 DESERT ROCK AIRSTRIP FUEL SPILL, NEVADA TEST SITE, NEVADA
}

\author{
U.S. Department of Energy \\ National Nuclear Security Administration \\ Nevada Operations Office \\ Las Vegas, Nevada
}

Controlled Copy No.:

Revision No.: 0

June 2002

Approved for public release; further dissemination unlimited. 


\section{POST-CLOSURE MONITORING REPORT FOR CORRECTIVE ACTION UNIT 329: \\ AREA 22 DESERT ROCK AIRSTRIP FUEL SPILL, NEVADA TEST SITE, NEVADA}

\footnotetext{
Approved by: Signature Approval Date: 6-6-02

Janet Appenzeller-Wing, Project Manager

Industrial Sites Project
}

Approved by:_Signature Approval Date: 6-6-02

Runore C. Wycoff, Division Director

Environmental Restoration Project 


\section{Table of Contents}

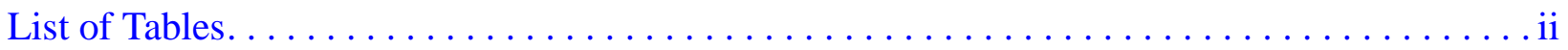

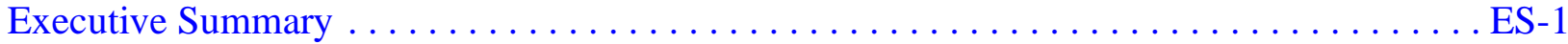

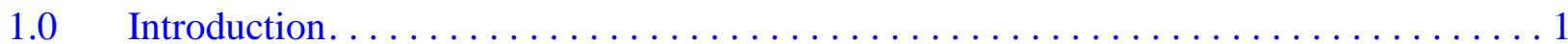

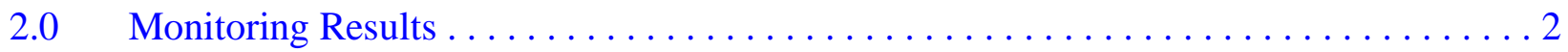

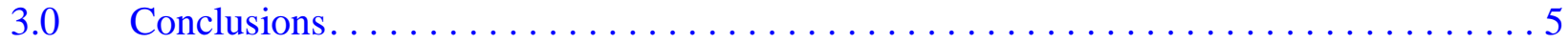

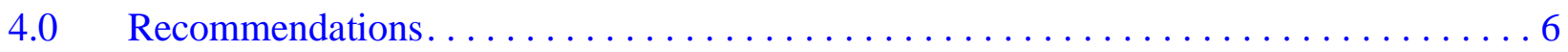

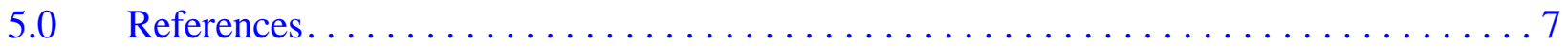

Appendix A - Analytical Data

Appendix B - NDEP Comment Responses 


\section{List of Tables}

Number

1-1 Samples Collected and Analyzed During the CAU 329 Monitoring Activities . . . . 1

2-1 Soil Gas Monitoring Sample Collection Results for Borehole DRA-0 . . . . . . . 3

2-2 Soil Gas Monitoring Sample Collection Results for Borehole DRA-3 . . . . . . 3

2-3 SVOC Sample Collection Results for Borehole DRA-0 . . . . . . . . . . 4 


\section{Executive Summary}

Post-closure monitoring of Corrective Action Unit 329 Area 22 Desert Rock Airstrip Fuel Spill, Nevada Test Site, Nevada, was completed on August 31, 2001. Post-closure monitoring for the site is scheduled annually for 5 years. The Corrective Action Unit consists of Corrective Action Site 22-44-01, Fuel Spill, and is located at the Nevada Test Site Area 22 Desert Rock Airstrip.

This was the first post-closure monitoring following establishment of baseline conditions in August 2000. The monitoring identified an increase in total petroleum hydrocarbons at all three depths sampled. Based on the analytical results, a rate of hydrocarbon degradation cannot be evaluated.

In order to determine degradation rates, additional data are required. It is recommended that additional samples be collected in August 2002 as specified in the current schedule. Data from the next sampling event will be summarized and provided in a post-closure monitoring report. 


\subsection{Introduction}

Annual post-closure monitoring of the Area 22 Desert Rock Airstrip Fuel Spill (Corrective Action Unit [CAU] 329, Corrective Action Site 22-44-01) was conducted on August 31, 2001. As specified in Table 1-1, the analyses included volatile organic compounds (VOCs), semivolatile organic compounds (SVOCs), total petroleum hydrocarbons (TPH) as Jet-A, oxygen, nitrogen, carbon dioxide, and methane. The annual monitoring was completed as specified in the CAU 329 Closure Report (DOE/NV, 2000).

Table 1-1

Samples Collected and Analyzed During the CAU 329 Monitoring Activities

\begin{tabular}{|c|c|c|}
\hline Borehole & $\begin{array}{c}\text { Soil-Gas Monitoring Point Depth } \\
\text { (ft bgs) }\end{array}$ & Analysis \\
\hline \hline DRA-0 & $40,80,120$ & $\begin{array}{c}\text { VOCs, SVOCs, TPH (as Jet-A), oxygen, } \\
\text { nitrogen, carbon dioxide, and methane }\end{array}$ \\
\hline DRA-3 & $50,75,120$ & $\begin{array}{c}\text { VOCs, TPH (as Jet-A), oxygen, nitrogen, } \\
\text { carbon dioxide, and methane }\end{array}$ \\
\hline
\end{tabular}

$\mathrm{ft}$ bgs $=$ Feet below ground surface 


\subsection{Monitoring Results}

The analytical results from Boreholes DRA-0 and DRA-3 are summarized in Table 2-1 and Table 2-2. Baseline analytical results from samples collected on August 30, 2000, are included for comparison. The SVOC results for Borehole DRA-0 are summarized in Table 2-3. The only SVOC detected above the method detection limit in August 2001 was naphthalene. There were no SVOC detected above the method detection limit in August 2000. Analytical results are included in Appendix A. 
Table 2-1

Soil Gas Monitoring Sample Collection Results for Borehole DRA-0

\begin{tabular}{|c|c|c|c|c|c|c|c|c|c|c|}
\hline \multirow{2}{*}{$\begin{array}{l}\text { Depth } \\
\text { (ft bgs) }\end{array}$} & \multicolumn{2}{|c|}{ TPH as Jet-A (PPMV) } & \multicolumn{2}{|c|}{ Oxygen } & \multicolumn{2}{|c|}{ Nitrogen } & \multicolumn{2}{|c|}{ Carbon Dioxide } & \multicolumn{2}{|c|}{ Methane } \\
\hline & $\begin{array}{c}\text { August } 30 \text {, } \\
2000\end{array}$ & $\begin{array}{c}\text { August } 31, \\
2001\end{array}$ & $\begin{array}{c}\text { August } 30, \\
2000\end{array}$ & $\begin{array}{c}\text { August } 31 \text {, } \\
2001\end{array}$ & $\begin{array}{c}\text { August } 30, \\
2000\end{array}$ & $\begin{array}{c}\text { August } 31 \text {, } \\
2001\end{array}$ & $\begin{array}{c}\text { August } 30 \text {, } \\
2000\end{array}$ & $\begin{array}{c}\text { August } 31, \\
2001\end{array}$ & $\begin{array}{l}\text { August } 30 \\
2000\end{array}$ & $\begin{array}{c}\text { August 31, } \\
2001\end{array}$ \\
\hline 40 & 4,500 & 11,000 & $5.0 \%$ & $2.6 \%$ & $94.8 \%$ & $83.9 \%$ & $3.1 \%$ & $13.6 \%$ & $<0.010 \%$ & $<0.010 \%$ \\
\hline 80 & 2,100 & 13,000 & $2.8 \%$ & $2.8 \%$ & $91.7 \%$ & $93.0 \%$ & $5.8 \%$ & $4.2 \%$ & $<0.010 \%$ & $<0.010 \%$ \\
\hline 120 & 6,000 & 7,800 & $2.7 \%$ & $2.7 \%$ & $90.0 \%$ & $93.5 \%$ & $6.7 \%$ & $3.8 \%$ & $<0.010 \%$ & $<0.010 \%$ \\
\hline
\end{tabular}

$\mathrm{ft}$ bgs $=$ Feet below ground surface

PPMV $=$ Parts per million volume

Table 2-2

Soil Gas Monitoring Sample Collection Results for Borehole DRA-3

\begin{tabular}{|c|c|c|c|c|c|c|c|c|c|c|}
\hline \multirow{2}{*}{$\begin{array}{l}\text { Depth } \\
\text { (ft bgs) }\end{array}$} & \multicolumn{2}{|c|}{ TPH as Jet-A (PPMV) } & \multicolumn{2}{|c|}{ Oxygen } & \multicolumn{2}{|c|}{ Nitrogen } & \multicolumn{2}{|c|}{ Carbon Dioxide } & \multicolumn{2}{|c|}{ Methane } \\
\hline & $\begin{array}{c}\text { August } 30, \\
2000\end{array}$ & $\begin{array}{c}\text { August } 31, \\
2001\end{array}$ & $\begin{array}{c}\text { August } 30, \\
2000\end{array}$ & $\begin{array}{c}\text { August } 31 \text {, } \\
2001\end{array}$ & $\begin{array}{c}\text { August } 30, \\
2000\end{array}$ & $\begin{array}{c}\text { August } 31, \\
2001\end{array}$ & $\begin{array}{c}\text { August } 30 \text {, } \\
2000\end{array}$ & $\begin{array}{c}\text { August } 31, \\
2001\end{array}$ & $\begin{array}{c}\text { August } 30, \\
2000\end{array}$ & $\begin{array}{c}\text { August } 31 \text {, } \\
2001\end{array}$ \\
\hline 50 & $<100$ & $<100$ & $6.6 \%$ & $7.4 \%$ & $94.2 \%$ & $89.3 \%$ & $2.1 \%$ & $3.3 \%$ & $<0.010 \%$ & $<0.010 \%$ \\
\hline 75 & $<100$ & $<100$ & $7.9 \%$ & $8.7 \%$ & $91.8 \%$ & $86.0 \%$ & $3.9 \%$ & $5.4 \%$ & $<0.010 \%$ & $<0.010 \%$ \\
\hline 120 & $<100$ & $<100$ & $6.4 \%$ & $12.1 \%$ & $92.0 \%$ & $87.5 \%$ & $2.2 \%$ & $0.41 \%$ & $<0.010 \%$ & $<0.010 \%$ \\
\hline
\end{tabular}


Table 2-3

SVOC Sample Collection Results for Borehole DRA-0

\begin{tabular}{|c|c|c|c||}
\hline \multirow{2}{*}{ Depth (ft bgs) } & \multicolumn{3}{|c|}{ Naphthalene } \\
\cline { 2 - 4 } & $\begin{array}{c}\text { Sample Results } \\
(\mu \mathrm{g} / \text { sample) }\end{array}$ & $\begin{array}{c}\text { Sample Volume } \\
\text { (Liters) }\end{array}$ & $\begin{array}{c}\text { Sample Concentration } \\
(\mu \mathrm{g} / \mathrm{L})\end{array}$ \\
\hline \hline 40 & 10 & 17.6 & 0.57 \\
\hline 80 & 44 & 17.0 & 2.59 \\
\hline 120 & 23 & 13.4 & 1.72 \\
\hline
\end{tabular}

$\mathrm{ft}$ bgs = Feet below ground surface

$\mu \mathrm{g} /$ sample $=$ Micrograms per sample

$\mu \mathrm{g} / \mathrm{L}=$ Micrograms per liter 


\subsection{Conclusions}

There was an increase in TPH concentrations at all three depths within Borehole DRA-0. The oxygen concentration at 40 feet $(\mathrm{ft})$ below ground surface (bgs) decreased. There was also an increase in carbon dioxide concentration at that depth. The decrease in oxygen concentrations and the increase in carbon dioxide concentration at the $40 \mathrm{ft}$ bgs level could be possible indicators of natural attenuation.

It is not possible to determine trends or biodegradation rates with the limited amount of data collected from the site.

The sample results from this first monitoring period did not correlate with the baseline results collected in August 2000. Additional samples will be collected and the results will be compared to previously collected samples to determine if the site was at equilibrium in August 2000. Continued annual monitoring will be conducted as specified in the Closure Report to determine trends at the site.

As natural attenuation occurs, the TPH concentrations should decrease. The TPH concentrations will be compared over successive monitoring events to determine trends and approximate rates. As natural attenuation occurs, oxygen will be consumed and carbon dioxide will be produced. The oxygen, nitrogen, and carbon dioxide concentrations will also be evaluated to determine if biodegradation is indicated. When all available oxygen has been consumed, methane-producing bacteria may continue the natural attenuation process so methane levels will be monitored as an additional possible indicator of natural attenuation.

The rate of decrease will be determined on the microbial populations, contaminant concentrations, available nutrients, and other environmental factors. Samples were collected and submitted for microbial analysis during closure activities. The results indicated that the microbial populations and nutrients were adequate for limited bioremediation (DOE/NV, 2000). Additional sampling for microbial analysis are not planned.

The site is currently inactive and the source of additional contamination was removed. It was determined during closure activities that the wetting front has stabilized. Monitoring of Borehole DRA-3 has not shown any indications of contamination. Contamination migration to the water table is not expected based on current site conditions. 


\subsection{Recommendations}

Monitoring of the site should be continued on an annual basis for the analytes identified in the Closure Report. 


\subsection{References}

U.S. Department of Energy, Nevada Operations Office. 2000. Closure Report for Corrective Action Unit 329: Area 22 Desert Rock Airstrip Fuel Spill, Nevada Test Site, Nevada, Rev. 0. Las Vegas, NV. 
Appendix A

\section{Analytical Data}

(51 Pages) 


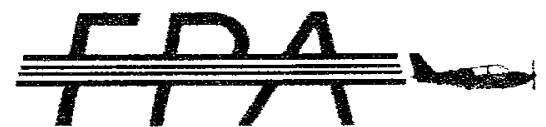

Field-Portable Analytical, Inc.

\title{
Report
}

\author{
IT Corporation \\ Nevada Test Site \\ Desert Rock Airport \\ August 31 ${ }^{\text {st }}, 2001$
}


IT Corporation contracted Field-Portable Analytical, Inc. $(F P A)$ to perform a soil gas investigation at the Desert Rock Airport in Mercury, Nevada. Monitoring was performed on August $31^{\text {st }}, 2000$. On-site analyses were performed for volatile organic compounds (VOC's) and using field-portable gas chromatography / Mass Spectrometry (GC/MS) and for atmospheric gases using field-portable gas chromatography (GC). In addition, offsite analyses were performed for semi-volatile organic compounds. This report presents the results obtained during this event.

Mr. David Curtis of FPA conducted the sampling and analysis for each event.

\section{Technical Approach}

The approach for this project consisted of both physical and chemical measurements.

- Flow measurements were performed to calculate the volume of air collected for SVOC analysis.

Chemical measurements were taken for Oxygen, Nitrogen, Methane, and Carbon Dioxide using field-portable gas chromatography (GC). Analysis for a specific list of 28 VOC's and Total Petroleum Hydrocarbons as Jet-A was performed using fieldportable gas chromatography / Mass Spectrometry (GC/MS). Samples were collected using PUF/XAD-2 sorbent cartridges and sent off-site for analysis of the standard list of 66 SVOC's.

\section{Sampling Locations}

The sampling locations and depths for this event were determined IT Corporation personnel 


\section{Chemical Measurements}

* Sampling System

Samples for on-site analysis were collected into 1 liter Tedlar bags. Each well was purged for two minutes at approximately 3 liters per minute prior to sample collection. The sample line was then attached to a high vacuum lung chamber sampler for collection of the bag samples.

Samples for off-site analysis were collected into PUF/XAD-2 sorbent cartridges. Each well was purged for two minutes at approximately 3 liters per minute prior to sample collection. The sample line was then attached to the sorbent cartridge for sample collection.

\section{- Analytical System (Micro GC)}

A P200H microchip gas chromatograph (GC), manufactured by Agilent Technologies, Wilmington, Delaware, was used for analysis of atmospheric gases for this event. The P200H contained two analytical modules. Each module consisted of a heated inlet, micromachined injection valve, column designed for Volatile Organic Compounds (VOC's), and a thermoconductivity detector. The GC contained an internal sampling pump which drew the sample through the individual sampling valves. Thus allowing each module to pull the necessary aliquot for analysis. The GC was controlled by laptop computers which also reduced and stored the data.

Module A of the GC contained a 10 meter Mol-Sieve column. Module B of contained an 8 meter Pora-Plot column. Table 1 shows the operating conditions of this GC's.

Table 1: GC Operating Conditions

\begin{tabular}{|l|c|c|}
\hline Instrument & Wiley Coyote & Chicken Hawk \\
\hline Detector Setting & High & High \\
\hline Module & A & A \\
\hline Column Temperature $\left({ }^{\circ} \mathrm{C}\right)$ & 110 & 55 \\
\hline Run Time (seconds) & 160 & 160 \\
\hline Sample Time (seconds) & 15 & 15 \\
\hline Inject Time (milliseconds) & 40 & 40 \\
\hline
\end{tabular}




\section{- Analytical System (GC/MS)}

FPA utilized an Inficon Hapsite GC/MS for analysis of VOC's and TPH as Jet-A during this event. This is a truly portable GC/MS designed specifically for the analysis of volatile compounds. The Hapsite is a full featured quadrupole GC/MS capable of meeting all of the EPA's stringent SW-846 QC criteria even though it weighs only 37 pounds and can be carried over the shoulder.

The Hapsite GC/MS uses a sampling wand with an internal pump to collect the sample. The sample is pulled into a sample loop with variable injection capabilities. The column is a 30 meter OV-1 with a 3 meter backflush column. The backflush column allows the volatile organic target compounds to get onto the column, then backflushes off the nontarget semivolatile compounds. This keeps the instrument free of contamination and eliminates the need to 'bake out' the contamination between analyses. The interface between the GC and MS is a methyl silicone membrane. This membrane allows organics to migrate through to the MS while sweeping most non-organics out through the vent.

By minimizing what gets into the MS, this instrument is able to utilize a chemical 'getter' pump rather than a mechanical pump. The getter pump maintains adequate vacuum for weeks at a time. It is very compact and allows the GC/MS to be used in a portable mode without the need to drag heavy mechanical pumps around.

The run time on the Hapsite GC/MS was about 22 minutes in order to obtain analysis for all the compounds involved with TPH as Jet-A. Since the column is isothermal and the unwanted compounds never reach the analytical column, there is no cool down time and the next analysis can be started immediately after the last for maximum throughput.

The Hapsite GC/MS co-injected an internal standard and a surrogate with every analysis. These compounds were used as additional QA/QC for each analysis. 


\section{Results Summary}

Samples from three depths of well DRAO and from three depths of well DRA3 were collected on August $31^{\text {st }}, 2001$. Table 2 shows the collection information for the six samples.

Table 2: Sample Collection

\begin{tabular}{||c|c|c|c|c|c|c||}
\hline Location & $\begin{array}{c}\text { Sample } \\
\text { Depth } \\
\text { Feet) }\end{array}$ & $\begin{array}{c}\text { VOC } \\
\text { Sample } \\
\text { Time }\end{array}$ & $\begin{array}{c}\text { SVOC } \\
\text { Sample } \\
\text { Time }\end{array}$ & $\begin{array}{c}\text { SVOC } \\
\text { Collection } \\
\text { Time }\end{array}$ & $\begin{array}{c}\text { SVOC } \\
\text { Collection } \\
\text { Flow }\end{array}$ & $\begin{array}{c}\text { SVOC } \\
\text { Collection } \\
\text { Volume }\end{array}$ \\
\hline DRAO \#1 & 120 & $12: 43$ & $12: 52$ & $30 \mathrm{~min}$ & $0.428 \mathrm{~L} / \mathrm{min} *$ & 13.4 Liters \\
\hline DRAO \#2 & 80 & $13: 37$ & $13: 41$ & $30 \mathrm{~min}$ & $0.568 \mathrm{~L} / \mathrm{min}$ & 17.0 Liters \\
\hline DRAO \#3 & 40 & $14: 17$ & $14: 21$ & $30 \mathrm{~min}$ & $0.585 \mathrm{~L} / \mathrm{min}$ & 17.6 Liters \\
\hline DRA3 \#B & 75 & $13: 17$ & NA & NA & NA & NA \\
\hline DRA3 \#C & 50 & $13: 25$ & NA & NA & NA & NA \\
\hline DRA3 \#E & 120 & $13: 53$ & NA & NA & NA & NA \\
\hline
\end{tabular}

*A pump exchange was performed during the collection of DRAO \#1. Two minutes of collection were performed at $0.703 \mathrm{~L} / \mathrm{min}$. The remaining 28 minutes of collection were performed at $0.428 \mathrm{~L} / \mathrm{min}$.

See Tables 3 thru 9 in the appendices for the VOC, Atmospheric Gas, and TPH as Jet-A results.

- SVOC Results

Results for the SVOC analyses will be sent as soon as they are received from the fixed laboratory. 
Quality Assurance/Quality Control

The following steps were taken to ensure the data collected during this analytical event were of usable quality:

Single Point Calibration (GC)

The GC was calibrated for Oxygen, Nitrogen, Methane, and Carbon Dioxide. A single point calibration was performed at the beginning of the day of analysis. In addition, a verification analysis was performed at the end of the day to ensure instrument stability. The verification analysis met acceptance criteria of $\pm 10 \%$ of the expected concentration.

Single Point Calibration (GC/MS)

The GC/MS was calibrated for TPH as Jet A. A single point calibration was performed at the beginning of the day of analysis.

* Five Point Calibration (GC/MS)

The GC/MS was calibrated for the select list of 28 compounds. A five point calibration was performed. The five concentration levels spanned the linear range of the instrument. The calibration met the acceptance criteria of $\pm 30 \%$ relative standard deviation (RSD).

There was a mid-level calibration check standard analyzed on the GC/MS at the beginning of the day of analysis. The check standard met the acceptance criteria of \pm $30 \%$ of the expected concentration.

The instrument tune was verified using to Bromofluorobenzene (BFB) at the beginning of the day of analysis. Standard SW-846 acceptance criteria were met.

In addition, an internal standard and a surrogate were co-injected with every analysis on the GC/MS. These compounds monitored any drift within the mass spectrometer. Acceptance criteria for the internal standard met SW-846 criteria of $50 \%$ to $200 \%$ recovery. Acceptance criteria for the surrogate met SW-846 criteria of $70 \%$ to $130 \%$ recovery. 
- Blanks

Blank samples were analyzed after calibrations were performed to ensure there was no carryover affecting samples. All blanks were within the acceptance criteria of no compound concentrations above the detection limit.

SVOC Analyses

SVOC analyses were performed by Performance Analytical. Quality assurance/quality control procedures followed standard methodology and will be documented with their results. 


\section{Conclusions}

Whole air samples were collected from wells DRAO \#1, DRAO \#2, DRAO \#3, DRA3 \#B, DRA3 \#C, and DRA3 \#E into 1 liter Tedlar bags. These samples were analyzed onsite for 28 VOC's, TPH as Jet-A, Oxygen, Nitrogen, Methane, and Carbon Dioxide. The data produced is of usable quality.

PUF/XAD-2 sorbent cartridges were collected from wells DRAO \#1, DRAO \#2, DRAO \#3, DRA3 \#B, DRA3 \#C, and DRA3 \#E. These samples were analyzed off-site for 66 SVOC's. The data produced is of usable quality. 


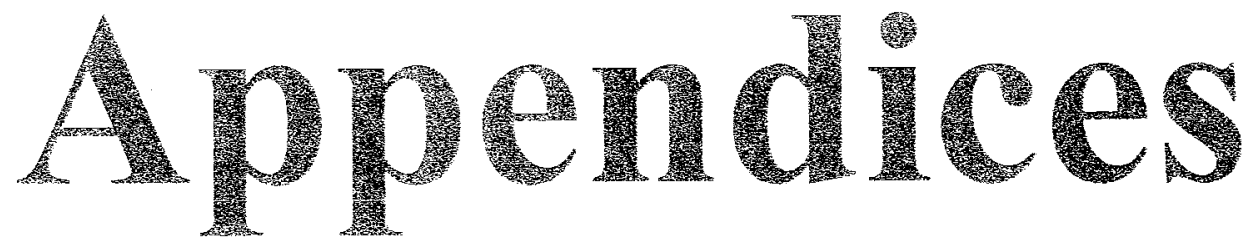




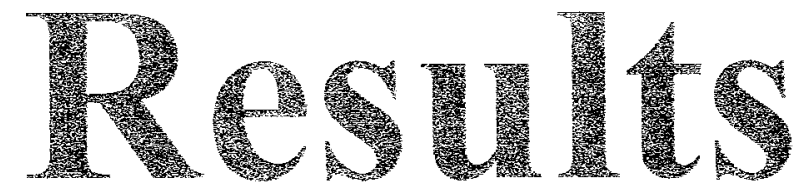




\section{$1 T$ Corporation}

\section{Nevada Test Site - Desert Rock Airport \\ Soil Gas Samples}

Sample Name:

File Number:

Sample Date:

Analysis Date:

Analysis Time:

Dilution Factor:

\author{
DRAO \#1 \\ D1083106/W1083104 \\ 31-Aug-01 \\ 31-Aug-01 \\ 12:53/13:54 \\ 1
}

\begin{tabular}{|c|c|}
\hline Compound & $\begin{array}{c}\begin{array}{c}\text { Concentration } \\
\text { (ppmv) }\end{array} \\
\end{array}$ \\
\hline Acetone & $<\quad 0.50$ \\
\hline Freon 11 & $<0.20$ \\
\hline 1,1-Dichloroethene & $<0.20$ \\
\hline Methylene Chloride & $<0.20$ \\
\hline Freon 113 & $<0.50$ \\
\hline trans-1,2-Dichloroethene & $<0.20$ \\
\hline 1,1-Dichloroethane & $<0.20$ \\
\hline MTBE & $<\quad 0.50$ \\
\hline cis-1,2-Dichloroethene & $<0.20$ \\
\hline n-Hexane & 9.4 \\
\hline Methyl Ethyl Ketone & $<0.50$ \\
\hline Chloroform & $<0.20$ \\
\hline 1,2-Dichloroethane & $<0.20$ \\
\hline 1,1,1-Trichloroethane & $<0.20$ \\
\hline Benzene & 5.5 \\
\hline Carbon Tetrachloride & $<0.20$ \\
\hline Trichloroethene & $<0.20$ \\
\hline 1,1,2-Trichloroethane & $<0.20$ \\
\hline Toluene & 14 \\
\hline 1,2-Dibromoethane & $<0.20$ \\
\hline Tetrachloroethene & $<0.20$ \\
\hline Chlorobenzene & $<\quad 0.20$ \\
\hline Ethyl Benzene & 5.1 \\
\hline m,p-Xylene & 14 \\
\hline Styrene & $<0.20$ \\
\hline o-Xylene & 15 \\
\hline 1,1,2,2-Tetrachloroethane & $<0.20$ \\
\hline Total Petroleum Hydrocarbons as Jet- $A$ & 7800 \\
\hline 1,3,5-tris(Trifluoromethyl)Benzene & Recovery (Accept. $=70 \%-130 \%)$ \\
\hline Compound & $\begin{array}{c}\begin{array}{c}\text { Concentration } \\
\text { (percent) }\end{array} \\
\end{array}$ \\
\hline Oxygen & $2.7 \%$ \\
\hline Nitrogen & $93.5 \%$ \\
\hline Methane & $<0.010 \%$ \\
\hline Carbon Dioxide & $3.8 \%$ \\
\hline
\end{tabular}


FULL SCAN Quantitation Report

Title: IT NTS Des Rock

$\begin{array}{lcccc}\text { FULL SCAN DATA : } & \text { C:IDATAVITNTSI31AUG01D1083106 } & & 0: 53: 04 & 1253 \\ \text { Acquired } & 9 / 1 / 01 & \text { at } & 1: 17: 20 & \end{array}$

Peak Search Method: C:IPROGRA 1WHAPSRUNMETHODUTNTSITNTS.LSM

PQUAN Library : C:IPROGRA - IHAPSRUNIMETHODUTNTSUTNTS

$\begin{array}{llll}\text { Last Calibration } & 8 / 25 / 01 & \text { at } & \end{array}$

Acquisition Method : C:IPROGRA -1HAAPSRUNMETHODIDEFAULT.FSM

Tune/Cal File : D1082501

Datafile title:

DRAO\#1

$W=R T+-(0: 20.00 / 2+R T * 0.005)$

Min Fit=0.750; Min Pur=0.200; Min Area $=25000$

Width $=2-40$ scans; $R e s=10$ scans; $N L M=1.0$

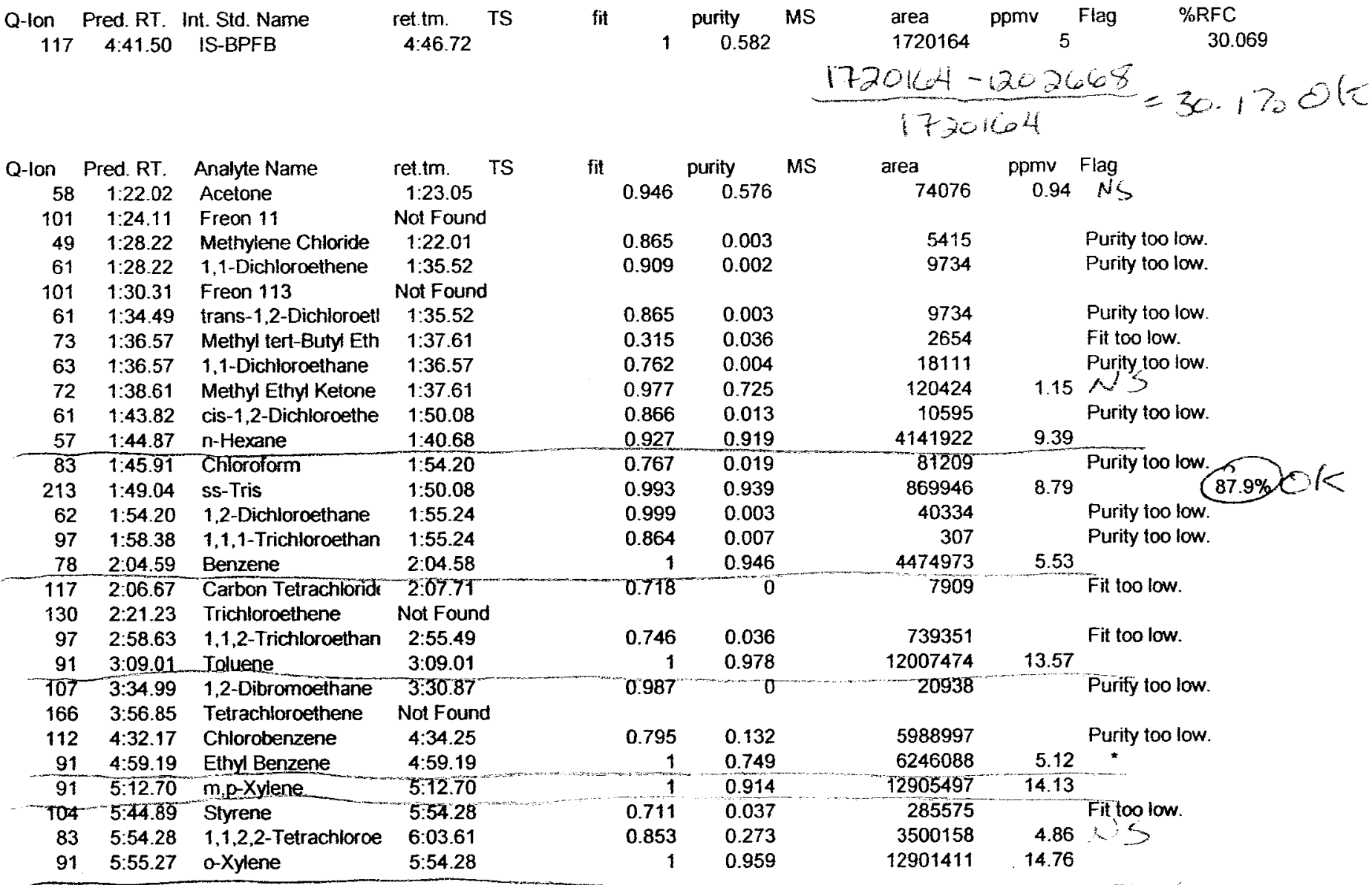




\section{Run: $09 / 01 / 01$ at $00: 53: 04$ DRAO \#1}

RTime $1: 15.75$ to $22: 25.57$

$\operatorname{Max}=42,587,443$

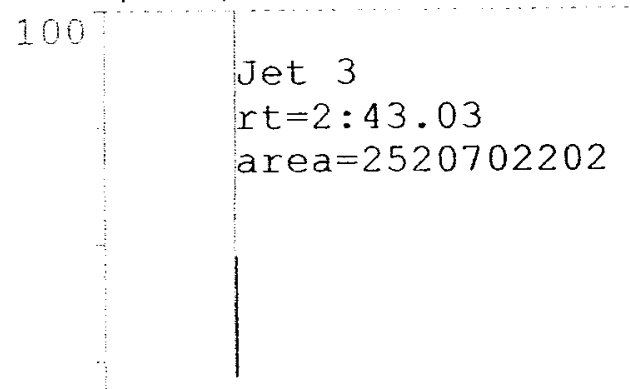

\section{Total $=2721965301 / 3+5675$}
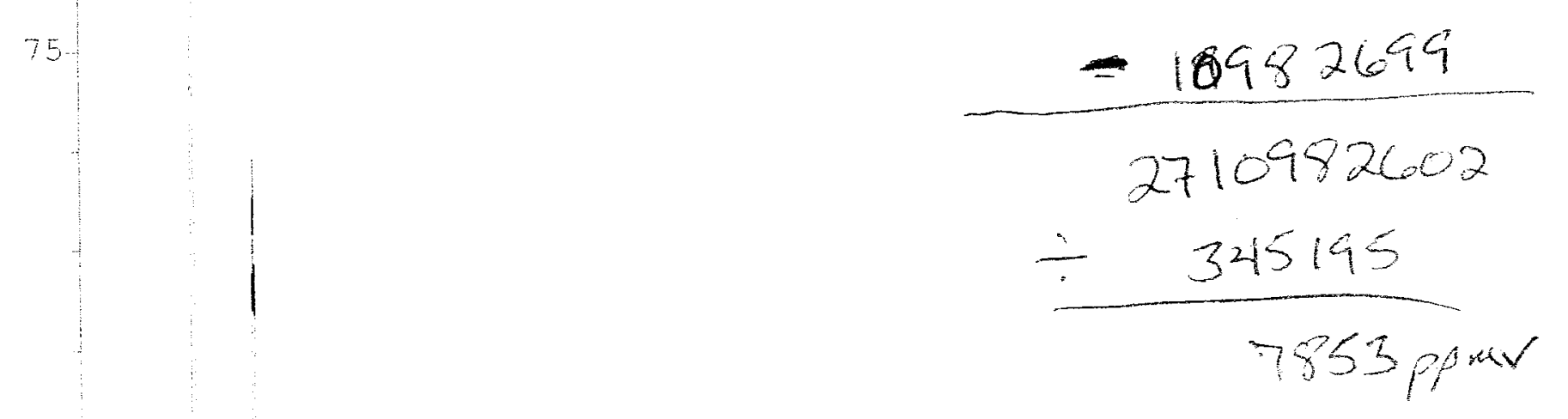

TIC

50

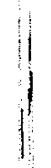

Jet 2

$r t=2: 20.18$

area $=192373090$

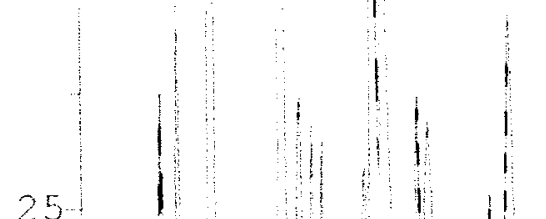

0

$1+2$

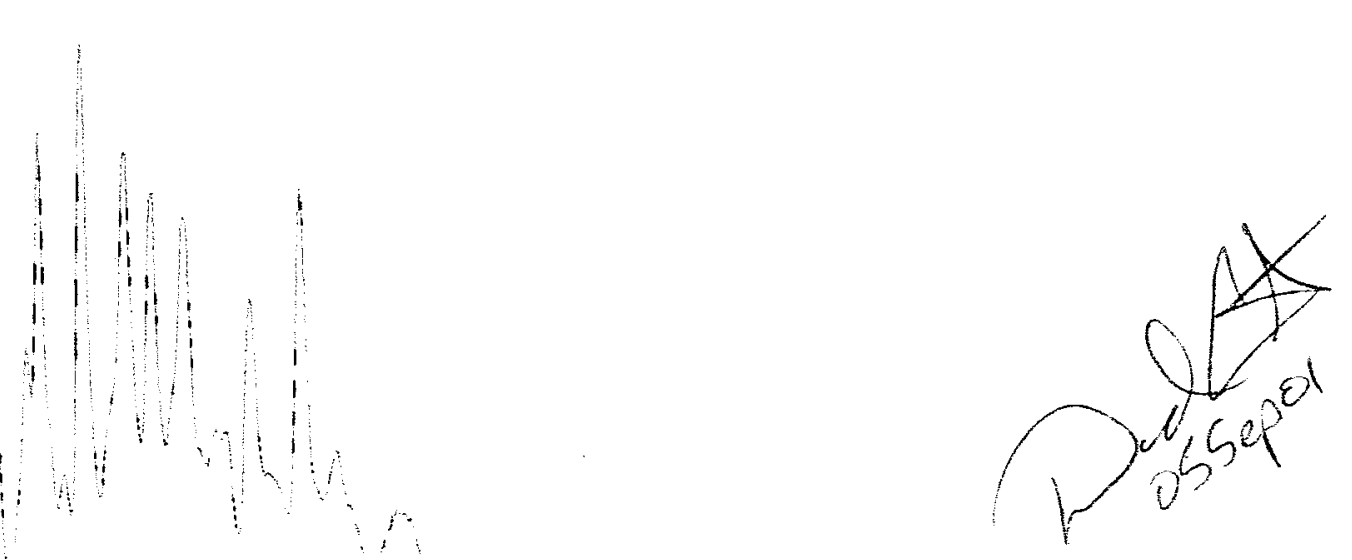

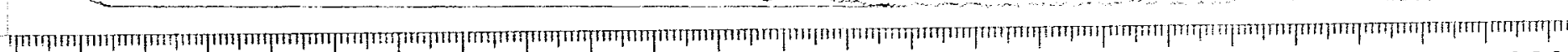

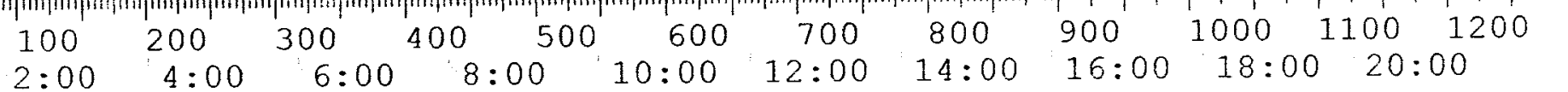




\section{IT Corporation \\ Nevada Test Site - Desert Rock Airport \\ Soil Gas Samples}

Sample Name:

File Number:

Sample Date:

Analysis Date:

Analysis Time:

Dilution Factor:
DRAO \#1 Duplicate

D1083107W1083105

31-Aug-01

31-Aug-01

13:17/13:58

1

Concentration

(ppmv)

$<0.50$

$<0.20$

$<0.20$

$<0.20$

$<0.50$

$<0.20$

$<0.20$

$<0.50$

$<0.20$

9.8

$<0.50$

$<0.20$

$<0.20$

$<0.20$

5.5

$<0.20$

$<0.20$

$<0.20$ 14

$<0.20$

$<0.20$

$<0.20$

5.1

14

$<0.20$

15

$<0.20$

9800

Recovery (Accept. $=70 \%-130 \%)$ :

$88.1 \%$

\begin{tabular}{|l|}
\hline Compound \\
\hline Oxygen \\
\hline Nitrogen \\
\hline Methane \\
\hline Carbon Dioxide \\
\hline
\end{tabular}

\section{Concentration}

(percent)

\begin{tabular}{c}
$2.7 \%$ \\
\hline $93.5 \%$ \\
$<0.010 \%$ \\
$3.7 \%$
\end{tabular}


FULL SCAN Quantitation Report

Title: IT NTS Des Rock

$\begin{array}{lcccc}\text { FULL SCAN DATA : } & \text { C:IDATAIITNTSI31AUG011D1083107 } & & \\ \text { Acquired } & 9 / 1 / 01 & \text { at } & 1: 17: 55 & 3 \\ \text { This Quantitation } & 9 / 1 / 01 & \text { at } & 1: 40: 52\end{array}$

Peak Search Method : C:IPROGRA -1IHAPSRUNIMETHODITNTSIITNTS.LSM

PQUAN Library : C:IPROGRA 1LAPSRUNIMETHODUTNTSUTNTS

$\begin{array}{llll}\text { Last Calibration } & 8 / 25 / 01 & \text { at } & \end{array}$

Acquisition Method : C:IPROGRA -1IHAPSRUNIMETHODIDEFAULT.FSM

Tune/Cal File : D1082501

Datainle title:

DRAO \#1 Duplicate

$W=R T+-\left(0: 20.00 / 2+R^{*} 0.005\right)$

Min Fit $=0.750$; Min Pur=0.200; Min Area $=25000$

Width $=2-40$ scans; Res $=10$ scans; $N L M=1.0$

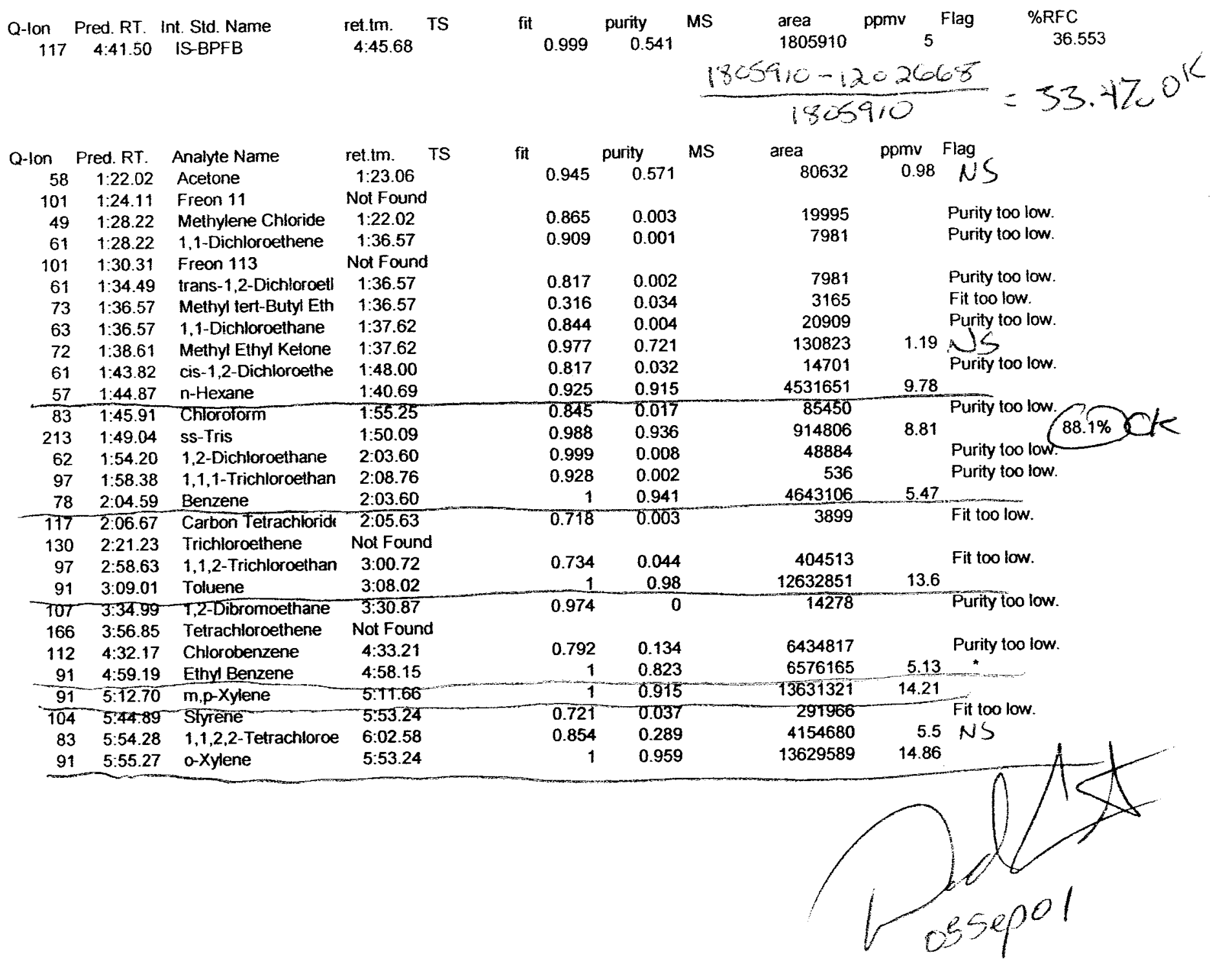


Run: $09 / 01 / 01$ at $01: 17: 55$

RTime $1: 15.76$ to $22: 27.67$

DRAO \#1 Duplicate

$\operatorname{Max}=42,689,559$

Jet 3

$\mathrm{rt}=2: 42.05$

area $=3170896102$

Total $=3382536634$

$\frac{-10982699}{3371553935}$

$\div 345195$

$75:$

50

Jet 2

$r t=2: 19)(14$

area $=202682361$

25

Jet 1

$r t=1: 23,06$

area $=9958171$

1

1

11

0

9767

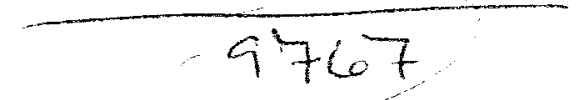




\section{Table 5 \\ IT Corporation \\ Nevada Test Site - Desert Rock Airport \\ Soil Gas Samples}

Sample Name:

File Number:

Sample Date:

Analysis Date:

Analysis Time:

Dilution Factor:
DRAO \#2

D1083108/W1083106

31-Aug-01

31-Aug-01

13:42/14:02

1

\begin{tabular}{|c|c|}
\hline Compound & $\begin{array}{c}\text { Concentration } \\
\text { (ppmv) }\end{array}$ \\
\hline Acetone & $<0.50$ \\
\hline Freon 11 & $<0.20$ \\
\hline 1,1-Dichloroethene & $<0.20$ \\
\hline Methylene Chloride & $<0.20$ \\
\hline Freon 113 & $<\quad 0.50$ \\
\hline trans-1,2-Dichloroethene & $<0.20$ \\
\hline 1,1-Dichloroethane & $<0.20$ \\
\hline MTBE & $<0.50$ \\
\hline cis-1,2-Dichloroethene & $<0.20$ \\
\hline n-Hexane & 14 \\
\hline Methyl Ethyl Ketone & $<0.50$ \\
\hline Chloroform & $<0.20$ \\
\hline 1,2-Dichloroethane & $<0.20$ \\
\hline 1,1,1-Trichloroethane & $<0.20$ \\
\hline Benzene & 3.8 \\
\hline Carbon Tetrachloride & $<0.20$ \\
\hline Trichloroethene & $<0.20$ \\
\hline 1,1,2-Trichloroethane & $<0.20$ \\
\hline Toluene & 0.60 \\
\hline 1,2-Dibromoethane & $<0.20$ \\
\hline Tetrachloroethene & $<0.20$ \\
\hline Chlorobenzene & $<0.20$ \\
\hline Ethyl Benzene & 6.2 \\
\hline$m, p-X y l e n e$ & 23 \\
\hline Styrene & $<0.20$ \\
\hline o-Xylene & 12 \\
\hline 1,1,2,2-Tetrachloroethane & $<0.20$ \\
\hline Total Petroleum Hydrocarbons as Jet- $A$ & 13,000 \\
\hline 1,3,5-tris(Trifluoromethyl)Benzene & Recovery (Accept. $=70 \%-130 \%)$ \\
\hline
\end{tabular}

\begin{tabular}{|l|c|}
\hline Compound & $\begin{array}{c}\text { Concentration } \\
\text { (ppmv) }\end{array}$ \\
\hline Oxygen & $2.8 \%$ \\
\hline Nitrogen & $93.0 \%$ \\
\hline Methane & $<0.010 \%$ \\
\hline Carbon Dioxide & $4.2 \%$ \\
\hline
\end{tabular}


FULL SCAN Quantitation Report

Title: IT NTS Des Rock

$\begin{array}{lccccc}\text { FULL SCAN DATA : } & \text { C:IDATAIITNTSI31AUG0101083108 } & & & \\ \text { Acquired } & 9 / 1 / 01 & \text { at } & 1: 42: 20 & 3: 4 & 2: 19: 03\end{array}$

Peak Search Method : C:IPROGRA 1HHAPSRUNIMETHODUTNTSITNTS.LSM

PQUAN Library : C:IPROGRA -1IHAPSRUNIMETHODVTNTSIITNTS

$\begin{array}{llll}\text { Last Calibration } & 8 / 25 / 01 & \text { at } & \end{array}$

Acquisition Method : C:IPROGRA -1LHAPSRUNMETHODIDEFAULT FSM

Tune/Cal File : D1082501

Datafile title:

DRAO \#2

$W=R T+-(0: 20.00 / 2+R T * 0.005)$

Min Fit=0.750; Min Pur $=0.200 ;$ Min Area $=25000$

Width $=2-40$ scans; $\operatorname{Res}=10$ scans; $N L M=1.0$

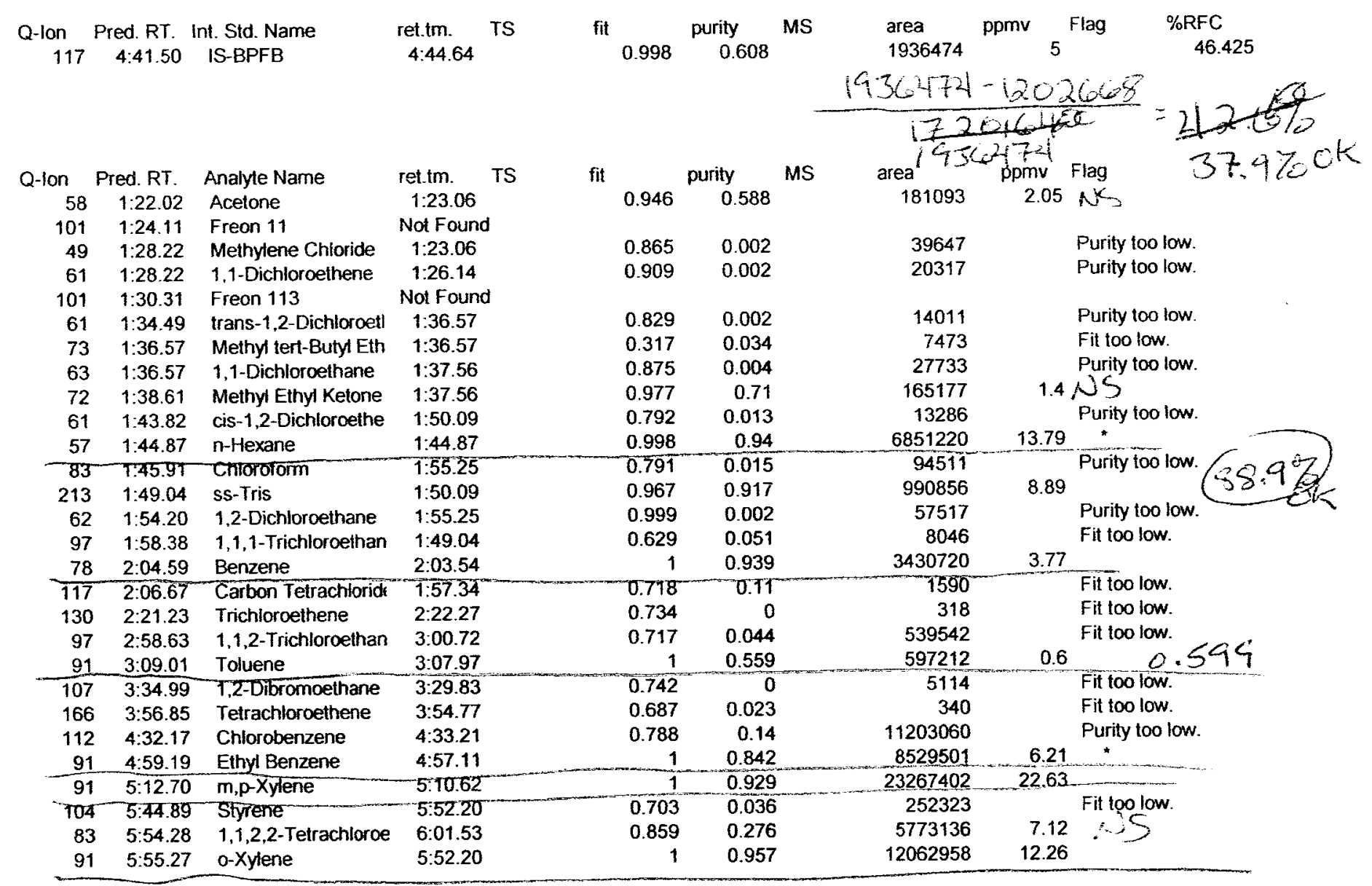

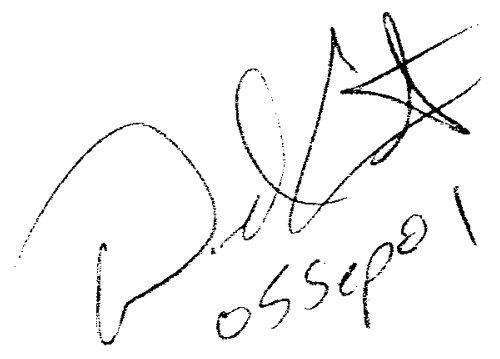


Run: $09 / 01 / 01$ at $01: 42: 20$

RTime $1: 15.76$ to $22: 30.74$

DRAO \#2 Duplicate

$\operatorname{Max}=55,203,372$

100

Jet 3

$r t=2: 43.03$

area $=4152389316$

75

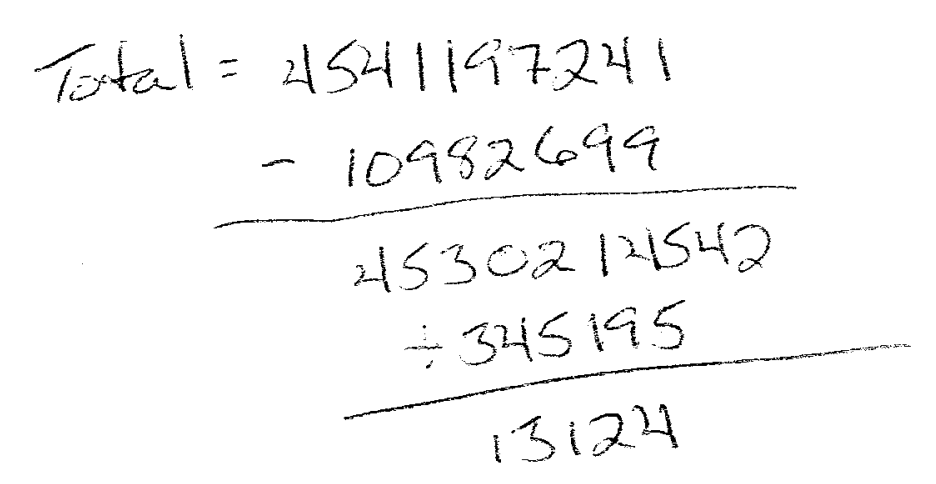

5

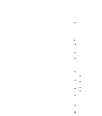

13124

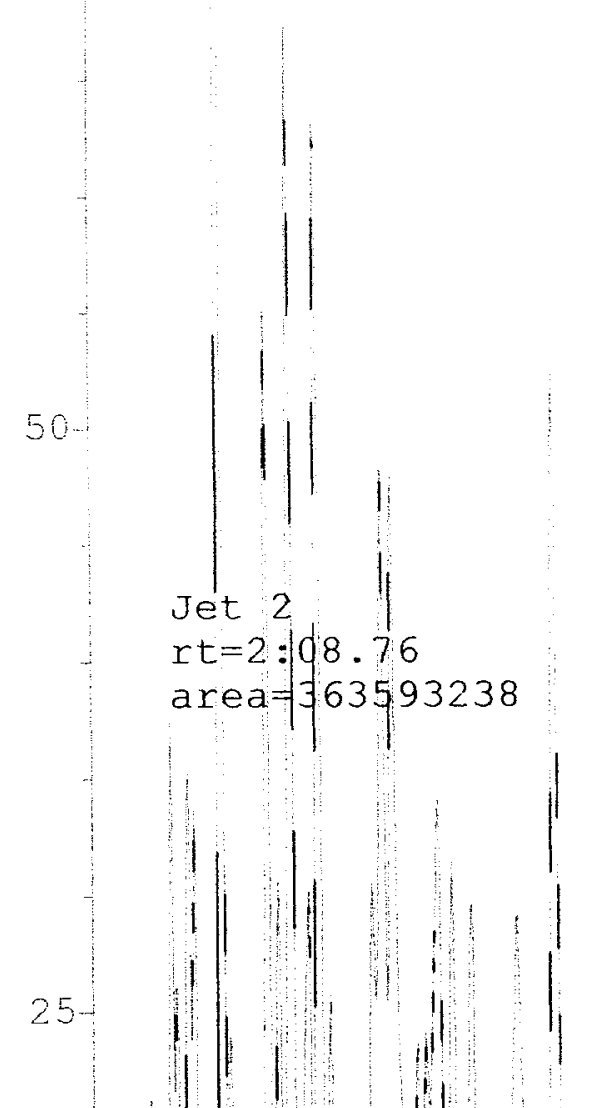

25
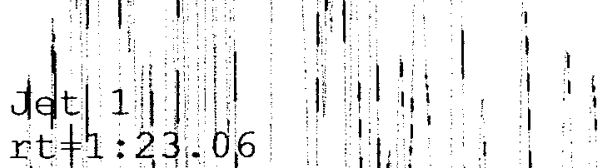

a)gqa-25214687

11
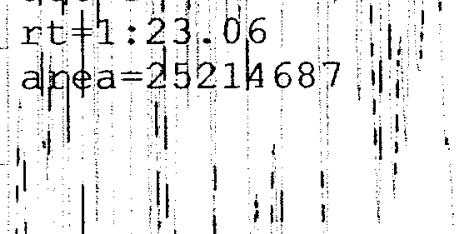

icate

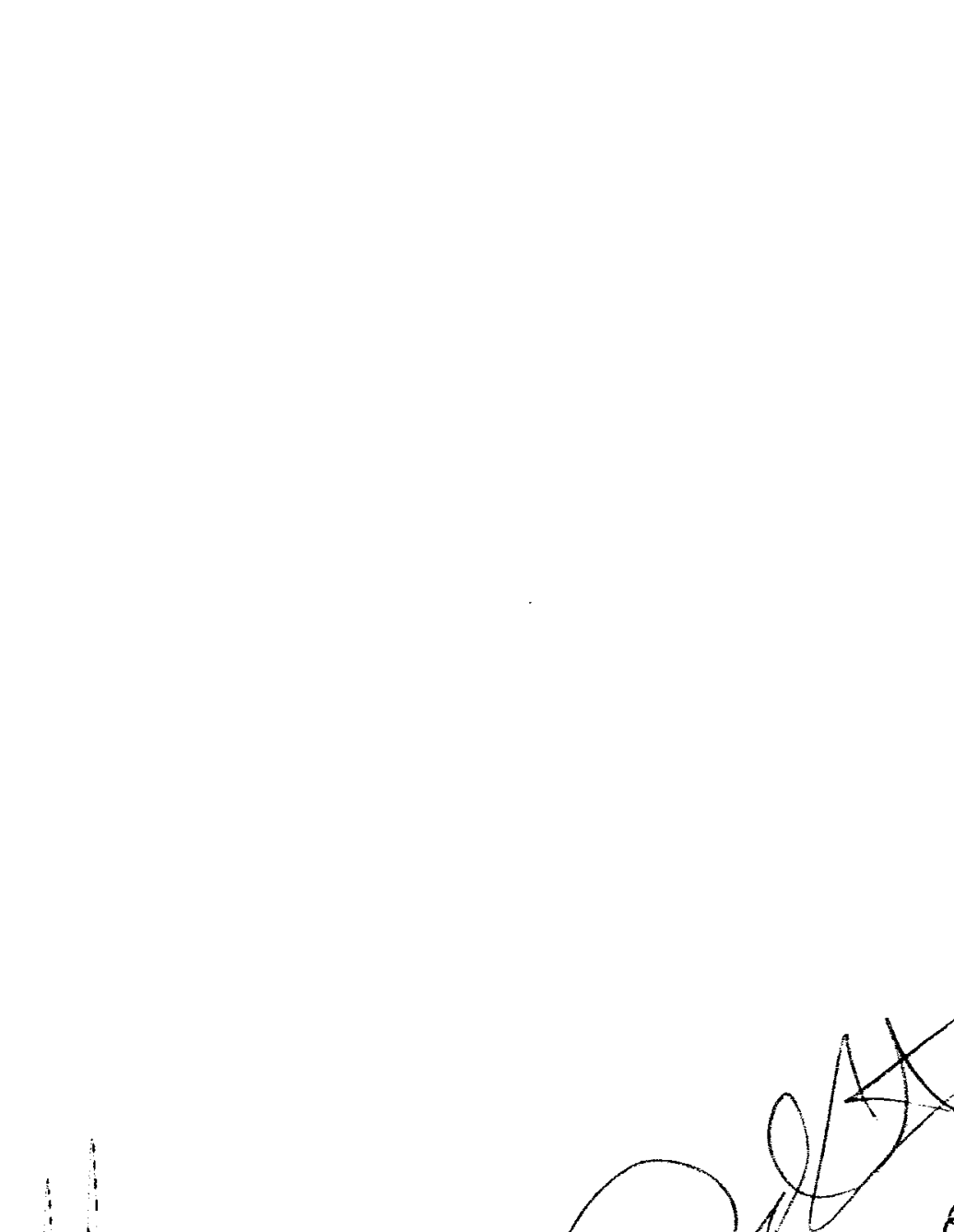




\section{Table 6}

\section{IT Corporation \\ Nevada Test Site - Desert Rock Airport \\ Soil Gas Samples}

Sample Name:

File Number:

DRAO \#3
D1083109/1083107
31-Aug-01
31-Aug-01
14:20/14:24
1

Sample Date:

Analysis Date:

Analysis Time:

Dilution Factor:

Concentration

(ppmv)

Acetone

Freon 11

1,1-Dichloroethene

Methylene Chloride

Freon 113

trans-1,2-Dichloroethene

1,1-Dichloroethane

MTBE

cis-1,2-Dichloroethene

n-Hexane

Methyl Ethyl Ketone

Chloroform

1,2-Dichloroethane

1,1,1-Trichloroethane

Benzene

Carbon Tetrachloride

Trichloroethene

1,1,2-Trichloroethane

Toluene

1,2-Dibromoethane

Tetrachloroethene

Chlorobenzene

Ethyl Benzene

m,p-Xylene

Styrene

o-Xylene

1,1,2,2-Tetrachloroethane

Total Petroleum Hydrocarbons as Jet-A

1,3,5-tris(Trifluoromethyl)Benzene

\begin{tabular}{|c|c|}
\hline \multicolumn{2}{|c|}{$\begin{array}{c}\text { Concentration } \\
\text { (ppmv) }\end{array}$} \\
\hline & 0.50 \\
\hline & 0.20 \\
\hline & 0.20 \\
\hline & 0.20 \\
\hline & 0.50 \\
\hline & 0.20 \\
\hline & 0.20 \\
\hline & 0.50 \\
\hline & 0.20 \\
\hline & 2.2 \\
\hline & 0.50 \\
\hline & 0.20 \\
\hline & 0,20 \\
\hline & 0.20 \\
\hline & 0.83 \\
\hline$<$ & 0.20 \\
\hline$<$ & 0.20 \\
\hline$<$ & 0.20 \\
\hline$<$ & 0.20 \\
\hline$<$ & 0.20 \\
\hline$<$ & 0.20 \\
\hline$<$ & 0.20 \\
\hline & 4.2 \\
\hline & 16 \\
\hline & 0.20 \\
\hline & 0.22 \\
\hline & 0.20 \\
\hline 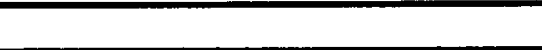 & 11,000 \\
\hline Recovery (Accept. $=70 \%-130 \%)$ & $91.2 \%$ \\
\hline
\end{tabular}

\begin{tabular}{|l|}
\hline Compound \\
\hline Oxygen \\
\hline Nitrogen \\
\hline Methane \\
\hline Carbon Dioxide \\
\hline
\end{tabular}

\section{Concentration}

(percent)

\begin{tabular}{l}
$2.6 \%$ \\
\hline $83.9 \%$ \\
\hline $0.010 \%$ \\
$13.6 \%$
\end{tabular}


FULL SCAN Quantitation Report

Title: IT NTS Des Rock

$\begin{array}{lclll}\text { FULL SCAN DATA : } & \text { C:IDATAIITTSI31AUG01D1083109 } & & 2: 20: 18 & 120 \\ \text { Acquired } & 9 / 1 / 01 & \text { at } & 2: 42: 58 & \end{array}$

Peak Search Method: C:IPROGRA-1IHAPSRUNMETHODUTNTSUTNTS.LSM

PQUAN Library : C:IPROGRA-1HAAPSRUNIMETHODUTNTSUTNTS

$\begin{array}{llll}\text { Last Calibration } & 8 / 25 / 01 & \text { at } & \end{array}$

Acquisition Method : C:IPROGRA -1LHAPSRUNMETHODIDEFAULT.FSM

Tune/Cal File : D1082501

Datafile title:

DRAO \#3

$W=R T+-(0.20 .00 / 2+R T * 0.005)$

Min Fit $=0.750$; Min Pur $=0.200$; Min Area $=25000$

Width $=2-40$ scans; Res $=10$ scans; NLM $=1.0$

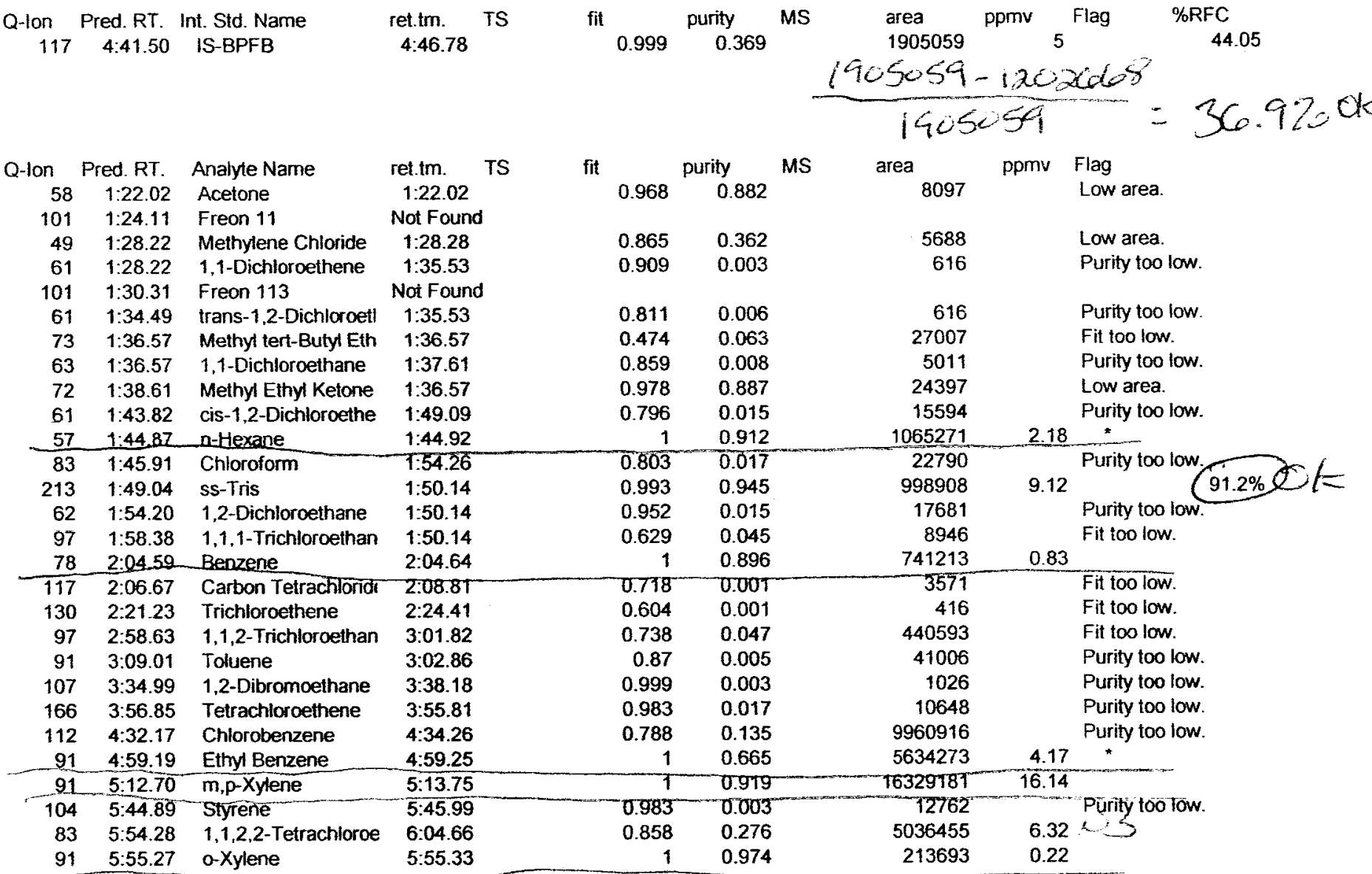

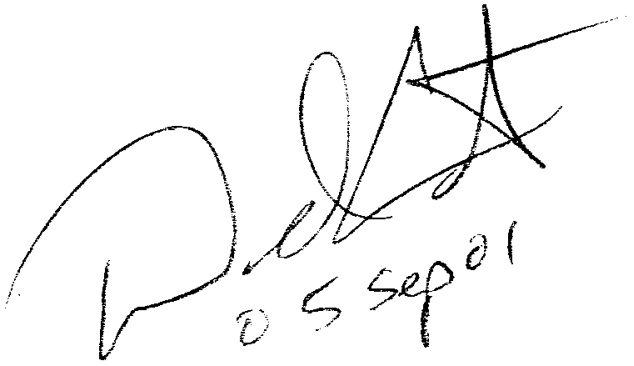


U11L villa $2 \cup y \pm 414$.

Time $1: 20.97$ to $22: 33.93$

Run: $09 / 01 / 01$ at $02: 20: 18$

DRAO \#3 Duplicate

$\operatorname{Max}=39,271,041$

TIC

$\begin{array}{ll}100: & \text { Jet } 3 \\ r t=2: 43.09 \\ \text { area }=3842537917\end{array}$

$$
\begin{aligned}
& \text { Total } \begin{array}{c}
3980282769 \\
-10982699
\end{array} \\
& \frac{396936 \times 070}{\div 345195} \\
& 11499
\end{aligned}
$$

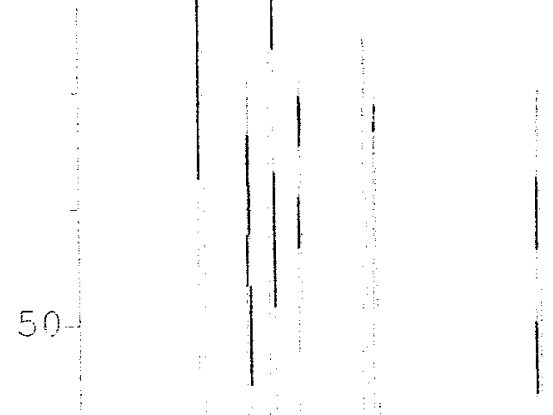




\section{Table 7}

\section{IT Corporation \\ Nevada Test Site - Desert Rock Airport \\ Soil Gas Samples}

Sample Name:

File Number:

Sample Date:

Analysis Date:

Analysis Time:

Dilution Factor:
DRA3 \#B

D1083111/W1083108

31-Aug-01

31-Aug-01

15:30/14:28

1

\begin{tabular}{|c|c|}
\hline Compound & $\begin{array}{c}\text { Concentration } \\
\text { (ppmv) }\end{array}$ \\
\hline Acetone & $<0.50$ \\
\hline Freon 11 & $<0.20$ \\
\hline 1,1-Dichloroethene & $<0.20$ \\
\hline Methylene Chloride & $<0.20$ \\
\hline Freon 113 & $<0.50$ \\
\hline trans-1,2-Dichloroethene & $<0.20$ \\
\hline 1,1-Dichloroethane & $<0.20$ \\
\hline MTBE & $<0.50$ \\
\hline cis-1,2-Dichloroethene & $<0.20$ \\
\hline n-Hexane & $<0.20$ \\
\hline Methyl Ethyl Ketone & $<0.50$ \\
\hline Chloroform & $<0.20$ \\
\hline 1,2-Dichloroethane & $<0.20$ \\
\hline 1,1,1-Trichloroethane & $<0.20$ \\
\hline Benzene & $<0.20$ \\
\hline Carbon Tetrachloride & $<0.20$ \\
\hline Trichloroethene & $<0.20$ \\
\hline 1,1,2-Trichloroethane & $<0.20$ \\
\hline Toluene & $<0.20$ \\
\hline 1,2-Dibromoethane & $<0.20$ \\
\hline Tetrachloroethene & $<0.20$ \\
\hline Chlorobenzene & $<\quad 0.20$ \\
\hline Ethyl Benzene & $<0.20$ \\
\hline m,p-Xylene & $<0.20$ \\
\hline Styrene & $<0.20$ \\
\hline o-Xylene & $<0.20$ \\
\hline 1,1,2,2-Tetrachloroethane & $<0.20$ \\
\hline Total Petroleum Hydrocarbons as Jet-A & $<100$ \\
\hline 1,3,5-tris(Trifluoromethyl)Benzene & Recovery (Accept. = 70\% - 130\%): \\
\hline Compound & $\begin{array}{c}\text { Concentration } \\
\text { (percent) }\end{array}$ \\
\hline Oxygen & $7.4 \%$ \\
\hline Nitrogen & $89.3 \%$ \\
\hline Methane & $<0.010 \%$ \\
\hline Carbon Dioxide & $3.3 \%$ \\
\hline
\end{tabular}


FULL SCAN Quantitation Report

Title: IT NTS Des Rock

FULL SCAN DATA : C:IDATAITNTSI31AUG01D1083111

$\begin{array}{lllll}\text { Acquired } & 9 / 1 / 01 & \text { at } & 3: 30: 43 & 15 \\ \text { This Quantitation } & 9 / 1 / 01 & \text { at } & 3: 45: 50\end{array}$

Peak Search Method: C:IPROGRA 1HHAPSRUNIMETHODUTNTSUTNTS.LSM

PQUAN Library : C:IPROGRA 1 1HAPSRUNIMETHODITNTSITNTS

$\begin{array}{llll}\text { Last Calibration } & 8 / 25 / 01 & \text { at } & \\ & & \end{array}$

Acquisition Method: C:IPROGRA 1IHAPSRUNIMETHODIDEFAULT.FSM

Tune/Cal File : D1082501

Datafile title:

DRA3 \#B

$W=R T+-(0: 20.00 / 2+R T * 0.005)$

Min Fit=0.750; Min Pur $=0.200 ;$ Min Area $=25000$

Width $=2-40$ scans; Res $=10$ scans; $N L M=1.0$

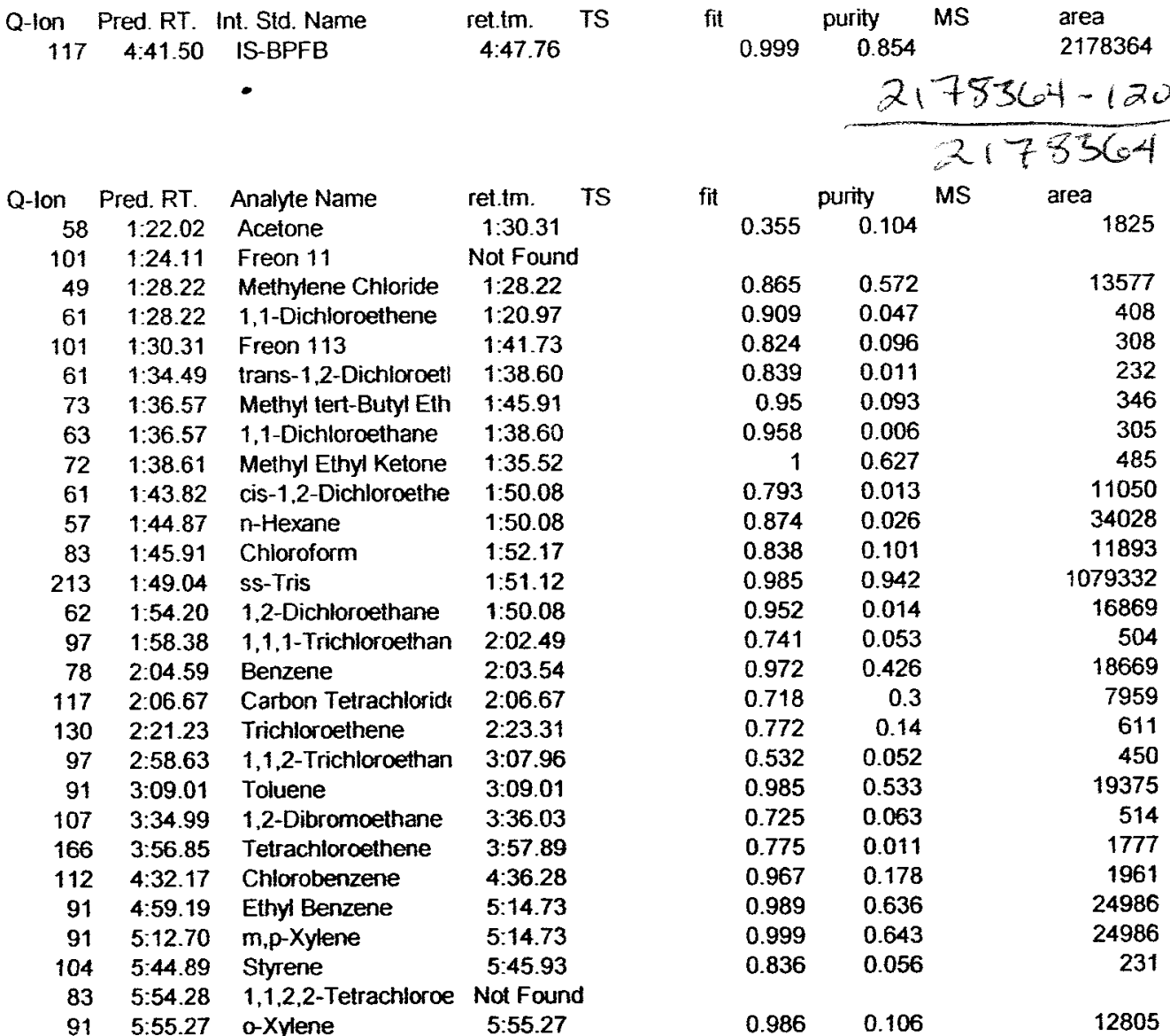

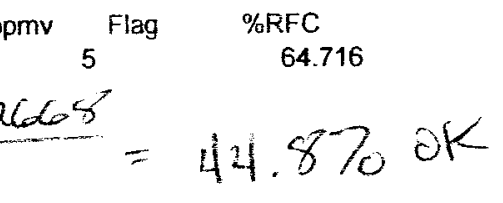

ppmv Flag

Fit too low.

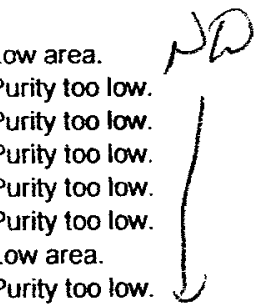

Purity 100 low.

Purity too low.

8.61

Purity too low $86.1 \%$ )

Fit too low.

Fit too low.

Purity too low.

Fit too low.

Low area.

Fit too low.

Purity too low.

Purity too low.

Low area.

Low area.

Purity too low

Purity too low.

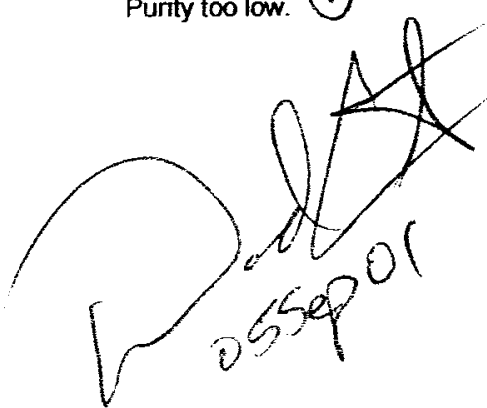




\section{Run: $09 / 01 / 01$ at $03: 30: 43$ DRA 3 \# $B$}

$\operatorname{Max}=15,140,289$

RTime $1: 11.58$ to $13: 43.95$

100

75

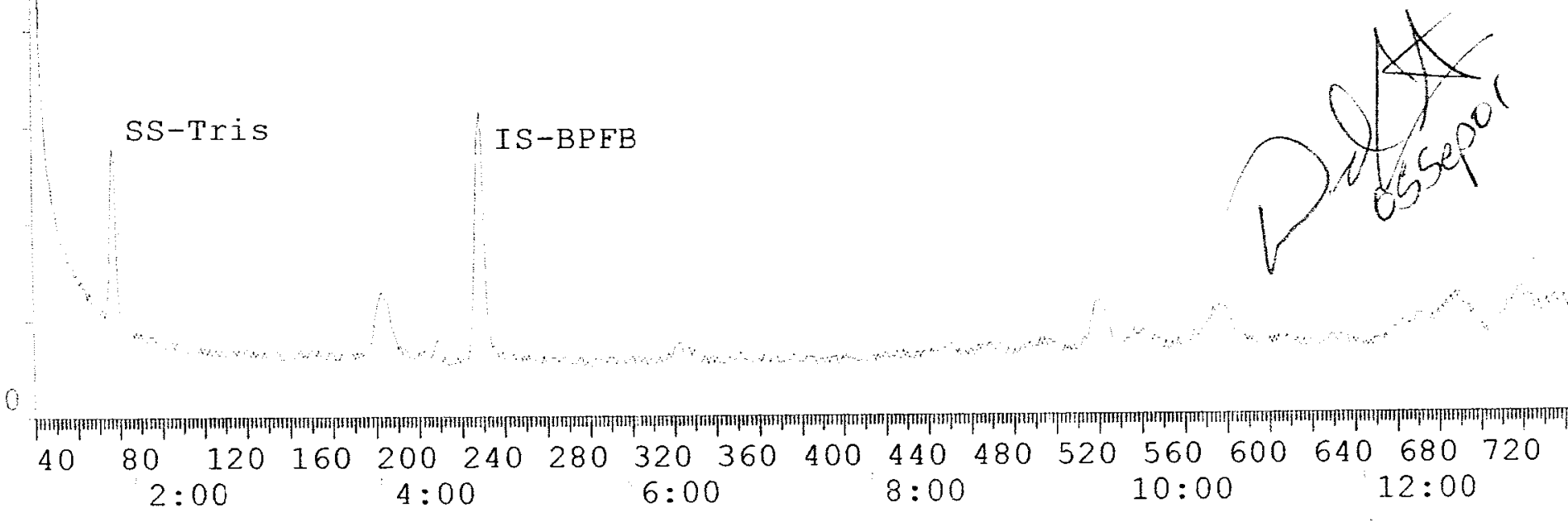




\section{Table 8 \\ IT Corporation \\ Nevada Test Site - Desert Rock Airport \\ Soil Gas Samples}

Sample Name:

File Number:

Sample Date:

Analysis Date:

Analysis Time:

Dilution Factor:

\author{
DRA3 \#C \\ D1083112N1083110 \\ 31-Aug-01 \\ 31-Aug-01 \\ 15:57/14:37 \\ 1
}

\begin{tabular}{|c|c|}
\hline Compound & $\begin{array}{c}\text { Concentration } \\
\text { (ppmv) }\end{array}$ \\
\hline Acetone & $<0.50$ \\
\hline Freon 11 & $<0.20$ \\
\hline 1,1-Dichloroethene & $<0.20$ \\
\hline Methylene Chloride & $<0.20$ \\
\hline Freon 113 & $<0.50$ \\
\hline trans-1,2-Dichloroethene & $<0.20$ \\
\hline 1,1-Dichloroethane & $<0.20$ \\
\hline MTBE & $<0.50$ \\
\hline cis-1,2-Dichloroethene & $<0.20$ \\
\hline n-Hexane & $<0.20$ \\
\hline Methyl Ethyl Ketone & $<0.50$ \\
\hline Chloroform & $<0.20$ \\
\hline 1,2-Dichloroethane & $<0.20$ \\
\hline 1,1,1-Trichloroethane & $<0.20$ \\
\hline Benzene & $<0.20$ \\
\hline Carbon Tetrachloride & $<0.20$ \\
\hline Trichloroethene & $<0.20$ \\
\hline 1,1,2-Trichloroethane & $<0.20$ \\
\hline Toluene & $<0.20$ \\
\hline 1,2-Dibromoethane & $<0.20$ \\
\hline Tetrachloroethene & $<0.20$ \\
\hline Chlorobenzene & $<0.20$ \\
\hline Ethyl Benzene & $<0.20$ \\
\hline m,p-Xylene & $<0.20$ \\
\hline Styrene & $<0.20$ \\
\hline o-Xylene & $<0.20$ \\
\hline 1,1,2,2-Tetrachloroethane & $<0.20$ \\
\hline Total Petroleum Hydrocarbons as Jet-A & $<100$ \\
\hline 1,3,5-tris(Trifluoromethyl)Benzene & Recovery (Accept. $=70 \%-130 \%$ ): \\
\hline Compound & $\begin{array}{c}\begin{array}{c}\text { Concentration } \\
\text { (ppmv) }\end{array} \\
\end{array}$ \\
\hline Oxygen & $8.7 \%$ \\
\hline Nitrogen & $86.0 \%$ \\
\hline Methane & $<0.010 \%$ \\
\hline Carbon Dioxide & $5.4 \%$ \\
\hline
\end{tabular}


FULL SCAN Quantitation Report

Title: IT NTS Des Rock

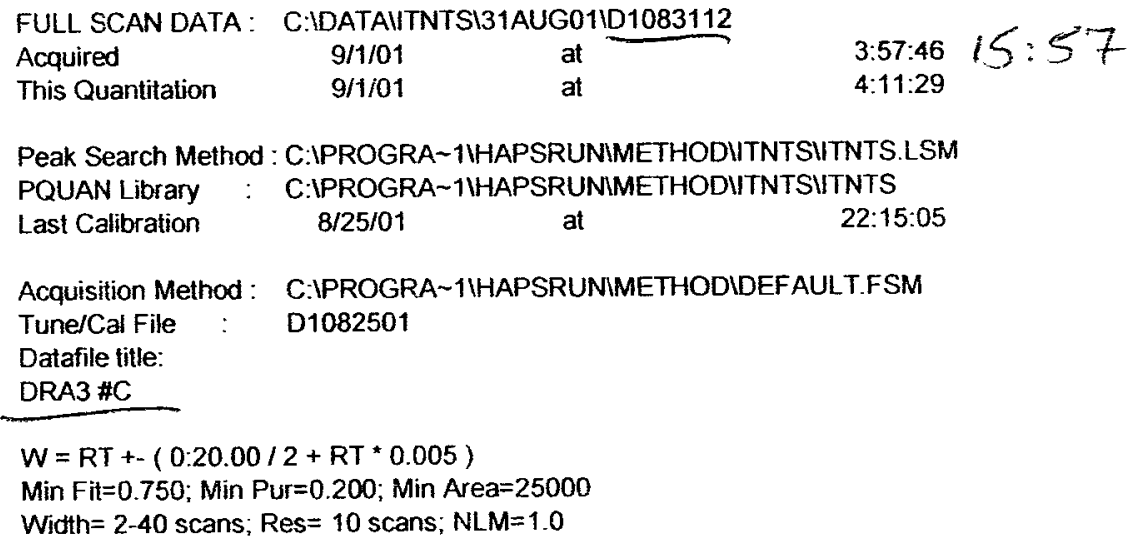

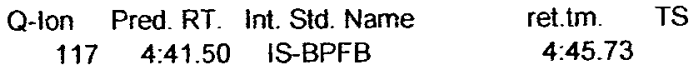

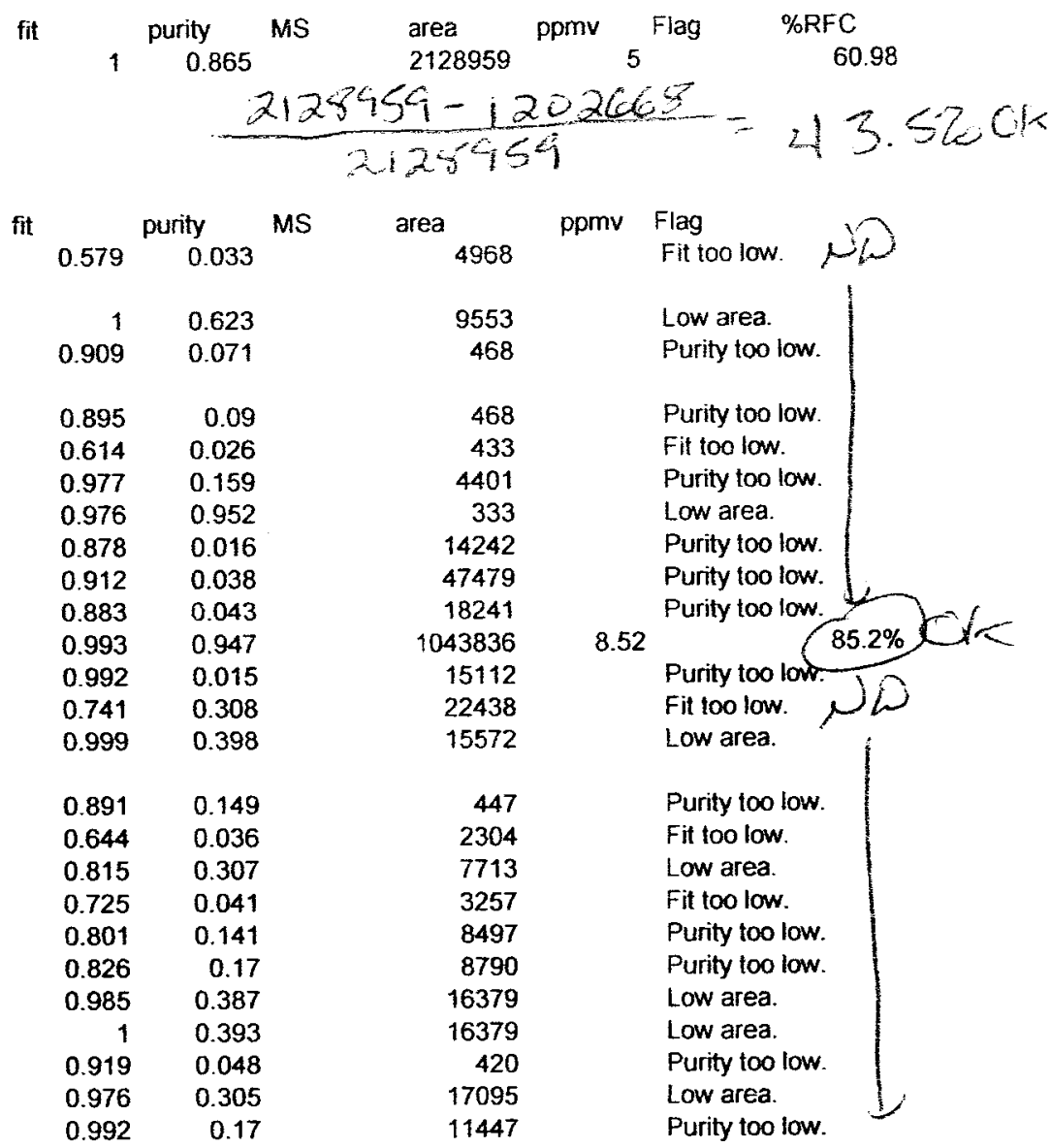

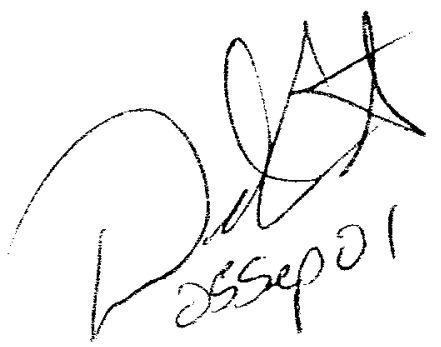


Run: $09 / 01 / 01$ at $03: 57: 46$

RTime $1: 11.69$ to $13: 17.97$

DRA 3 \#C

$\operatorname{Max}=20,927,953$

\section{0}

100

75

50

25

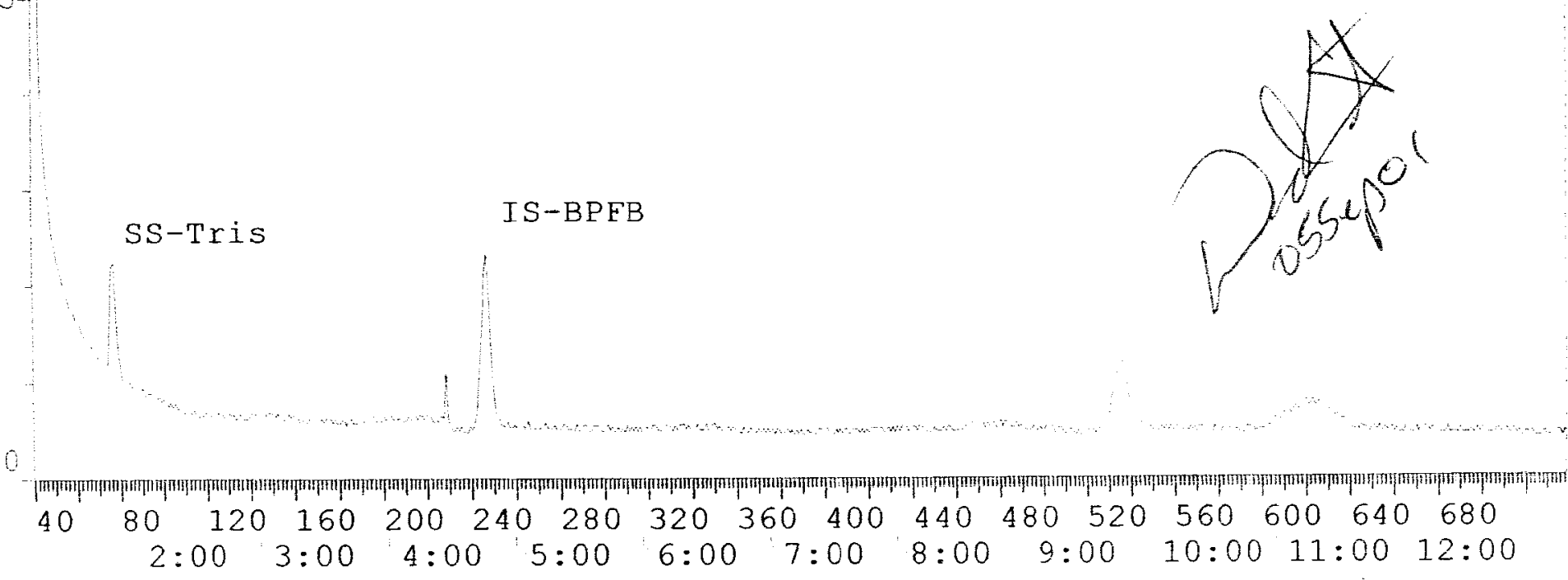




\section{Table 9}

\section{IT Copporation \\ Nevada Test Site - Desert Rock Airport \\ Soil Gas Samples}

Sample Name:

File Number:

Sample Date:

Analysis Date:

Analysis Time:

Dilution Factor:
DRA3 \#E

D1083113/W1083111

31-Aug-01

31-Aug-01

16:12/14:41

1

\begin{tabular}{|c|c|}
\hline Compound & $\begin{array}{c}\text { Concentration } \\
\text { (ppmv) }\end{array}$ \\
\hline Acetone & $<0.50$ \\
\hline Freon 11 & $<0.20$ \\
\hline 1,1-Dichloroethene & $<0.20$ \\
\hline Methylene Chloride & $<0.20$ \\
\hline Freon 113 & $<0.50$ \\
\hline trans-1,2-Dichloroethene & $<0.20$ \\
\hline 1,1-Dichloroethane & $<0.20$ \\
\hline MTBE & $<\quad 0.50$ \\
\hline cis-1,2-Dichloroethene & $<0.20$ \\
\hline n-Hexane & $<0.20$ \\
\hline Methyl Ethyl Ketone & $<0.50$ \\
\hline Chloroform & $<0.20$ \\
\hline 1,2-Dichloroethane & $<0.20$ \\
\hline 1,1,1-Trichloroethane & $<0.20$ \\
\hline Benzene & $<0.20$ \\
\hline Carbon Tetrachloride & $<0.20$ \\
\hline Trichloroethene & $<0.20$ \\
\hline 1,1,2-Trichloroethane & $<0.20$ \\
\hline Toluene & $<0.20$ \\
\hline 1,2-Dibromoethane & $<0.20$ \\
\hline Tetrachloroethene & $<0.20$ \\
\hline Chlorobenzene & $<0.20$ \\
\hline Ethyl Benzene & $<0.20$ \\
\hline$m, p$-Xylene & $<0.20$ \\
\hline Styrene & $<0.20$ \\
\hline o-Xylene & $<0.20$ \\
\hline 1,1,2,2-Tetrachloroethane & $<0.20$ \\
\hline Total Petroleum Hydrocarbons as Jet-A & $<100$ \\
\hline 1,3,5-tris(Trifluoromethyl)Benzene & Recovery (Accept. $=70 \%-130 \%)$ \\
\hline
\end{tabular}

\begin{tabular}{|l|}
\hline Compound \\
\hline Oxygen \\
\hline Nitrogen \\
\hline Methane \\
\hline Carbon Dioxide \\
\hline
\end{tabular}

Concentration

(percent)

$12.1 \%$

$87.5 \%$

$<0.010 \%$

$0.41 \%$ 
FULL SCAN Quantitation Report

Title: IT NTS Des Rock

$\begin{array}{lcllll}\text { FULL SCAN DATA : } & \text { C:IDATAITNTSI31AUGO1D1083113 } & & \\ \text { Acquired } & 9 / 1 / 01 & \text { at } & 4: 12: 07 & 1 & \\ \text { This Quantitation } & 9 / 1 / 01 & \text { at } & 4: 25: 40 & \end{array}$

Peak Search Method: C:IPROGRA -1LHAPSRUMMETHODUTNTSUTNTS.LSM

PQUAN Library : C:IPROGRA - 1HHAPSRUNIMETHODUTNTSUTNTS

$\begin{array}{llll}\text { Last Calibration } & 8 / 25 / 01 & \text { at } & \end{array}$

Acquisition Method: C:IPROGRA -1VHAPSRUNMETHODIDEFAULT.FSM

Tune/Cal File : D1082501

Datafile title:

DRA3 \#E

$W=R T+-(0: 20.00 / 2+R T * 0.005)$

Min Fit $=0.750$; Min Pur $=0.200 ;$ Min Area $=25000$

Width $=2-40$ scans; $R e s=10$ scans; $N$ ML $=1.0$ $\begin{array}{cccc}\text { Q-Ion } & \text { Pred. RT. Int. Std. Name } & \text { ret.tm. } & \text { TS } \\ 117 & 4: 41.50 & \text { IS-BPFB } & \mathbf{4 : 4 5 . 6 8}\end{array}$
Q-!on

\begin{tabular}{|c|c|c|c|}
\hline & Pred. RT. & Analyte Name & ret.tm. \\
\hline 58 & $1: 22.02$ & Acelone & 1:20.97 \\
\hline 101 & $1: 24.11$ & Freon 11 & Not Found \\
\hline 49 & $1: 28.22$ & Methylene Chloride & $1: 28.22$ \\
\hline 61 & $1: 28.22$ & 1,1-Dichloroethene & $1: 35.53$ \\
\hline 101 & $1: 30.31$ & Freon 113 & Not Found \\
\hline 61 & $1: 34.49$ & trans-1,2-Dichloroefl & $1: 35.53$ \\
\hline 73 & $1: 36.57$ & Methyl tert-Butyl Eth & $1: 36.57$ \\
\hline 63 & $1: 36.57$ & 1.1-Dichloroethane & Not Found \\
\hline 72 & $1: 38.61$ & Methyl Ethyl Ketone & $1: 44.86$ \\
\hline 61 & $1: 43.82$ & cis-1,2-Dichloroethe & 1.49 .04 \\
\hline 57 & $1: 44.87$ & n-Hexane & $1: 50.08$ \\
\hline 83 & $1: 45.91$ & Chloroform & $1: 43.82$ \\
\hline 213 & $1: 49.04$ & ss-Tris & $1: 50.08$ \\
\hline 62 & $1: 54.20$ & 1,2-Dichlorcethane & $1: 50.08$ \\
\hline 97 & $1: 58.38$ & 1.1.1-Trichloroethan & $2: 00.46$ \\
\hline 78 & $2: 04.59$ & Benzene & $1: 58.37$ \\
\hline 117 & $2: 06.67$ & Carbon Tetrachlorid & $2: 11.89$ \\
\hline 130 & $2: 21.23$ & Trichloroethene & $2: 21.22$ \\
\hline 97 & $2: 58.63$ & 1,1,2-Trichloroethan & Not Found \\
\hline 91 & $3: 09.01$ & Toluene & $3: 08.02$ \\
\hline 107 & $3: 34.99$ & 1,2-Dibromoethane & $3: 38.12$ \\
\hline 166 & $3: 56.85$ & Tetrachloroethene & Not Found \\
\hline 112 & $4: 32.17$ & Chlorobenzene & $4: 42.55$ \\
\hline 91 & $4: 59.19$ & Ethyl Benzene & $5: 12.70$ \\
\hline 91 & $5: 12.70$ & $m, p-X y l e n e$ & $5: 12.70$ \\
\hline 104 & $5: 44.89$ & Styrene & $5: 41.76$ \\
\hline 83 & $5: 54.28$ & 1,1,2,2-Tetrachloroe & Not Found \\
\hline 91 & $5: 55.27$ & o-xylene & $5: 54.22$ \\
\hline
\end{tabular}

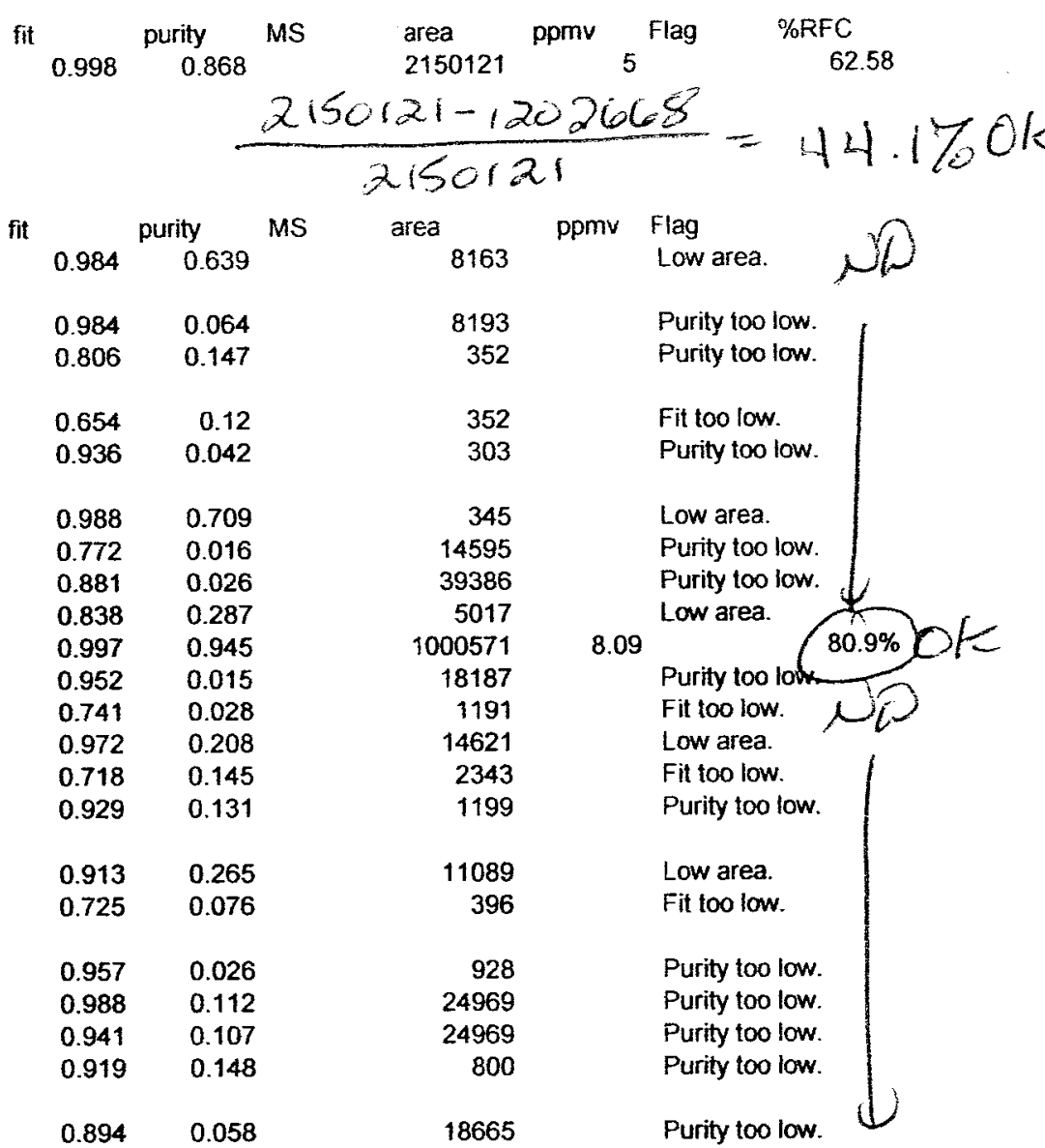

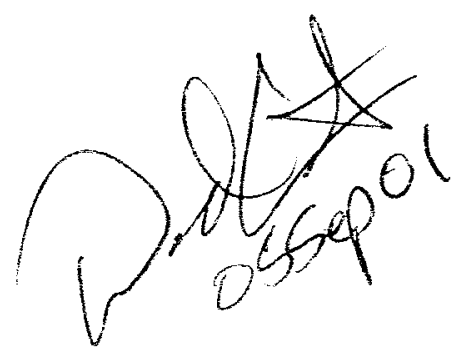




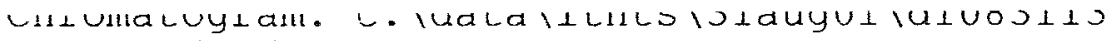

Run: 09/01/01 at 04:12:07

Dualls

DRA3 \#E

$\operatorname{Max}=27,204,797$

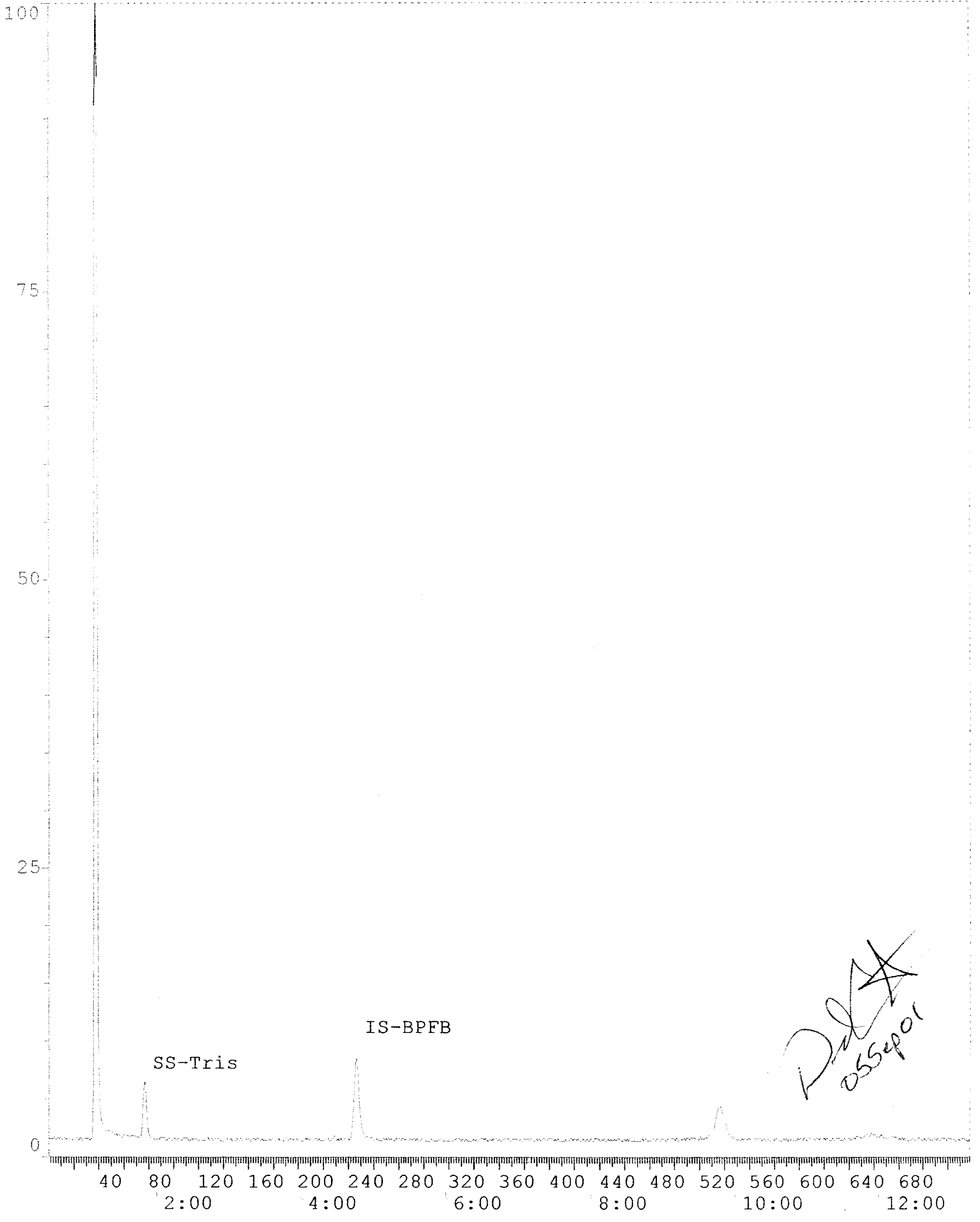


Calibrations 
FULL SCAN Quantitation Report

Tille: IT NTS Des Rock

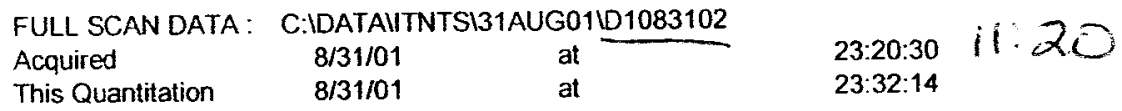

Peak Search Method: C:IPROGRA 1IHAPSRUNIMETHODUTNTSUTNTS.LSM

PQUAN Library : C:IPROGRA 1HHAPSRUNIMETHODITNTSITNTS

$\begin{array}{lll}\text { Last Calibration } & 8 / 25 / 01 & \text { at }\end{array}$

Acquisition Method : C:IPROGRA 1 1HAPSRUNIMETHODIDEFAULT.FSM

Tune/Cal File : $\quad$ D1082501

Datafile title:

10 ppmv ITNTS Slandard

$W=R T+-(0: 20.00 / 2+R T * 0.005)$

Min Fit $=0.750 ;$ Min Pur $=0.200$; Min Area $=25000$

Width $=2-40$ scans; Res $=10$ scans; $N L M=1.0$

$\begin{array}{cc}\text { Q-Ion } & \text { Pred. RT. Int. Std. Name } \\ 117 \quad 4: 41.50 & \text { IS-BPFB }\end{array}$ ret.tm. TS

$4: 40.51$ fit purity MS area

1202668 ppmv ${ }_{5}$ Flag
\%RFC $-9.061$

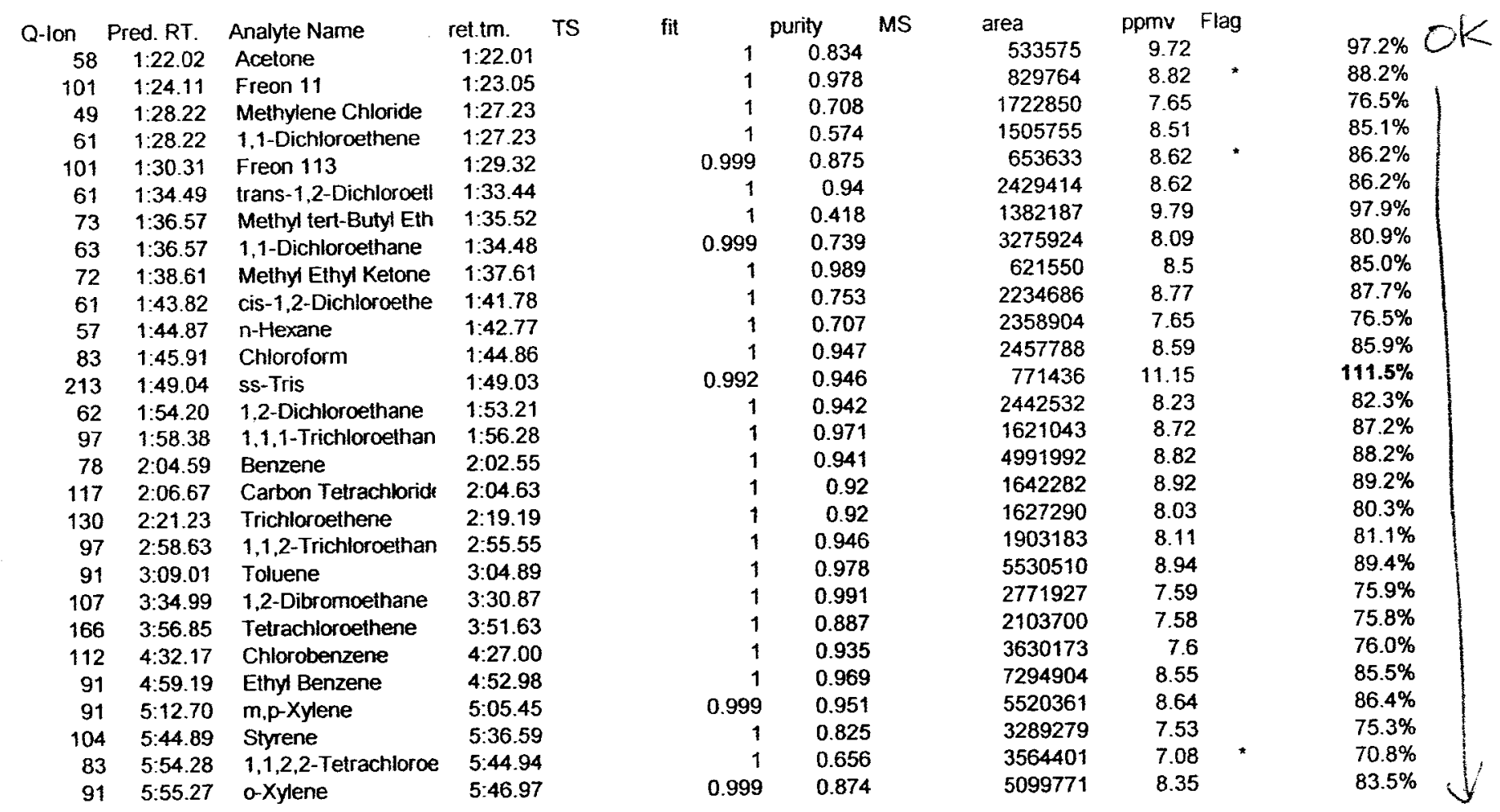

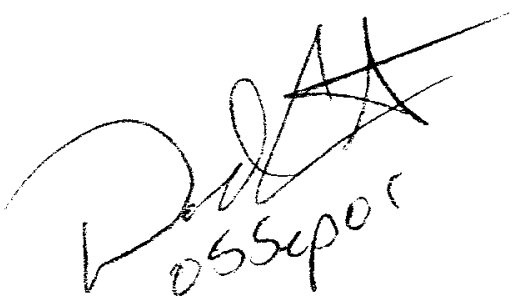




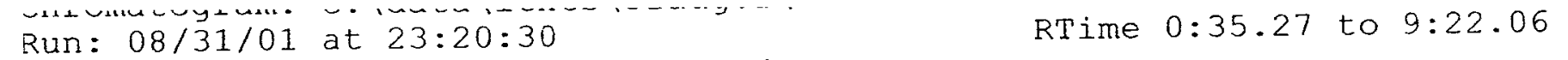

10 ppmv ITNTS standard

$\operatorname{Max}=5,418,308$

TIC

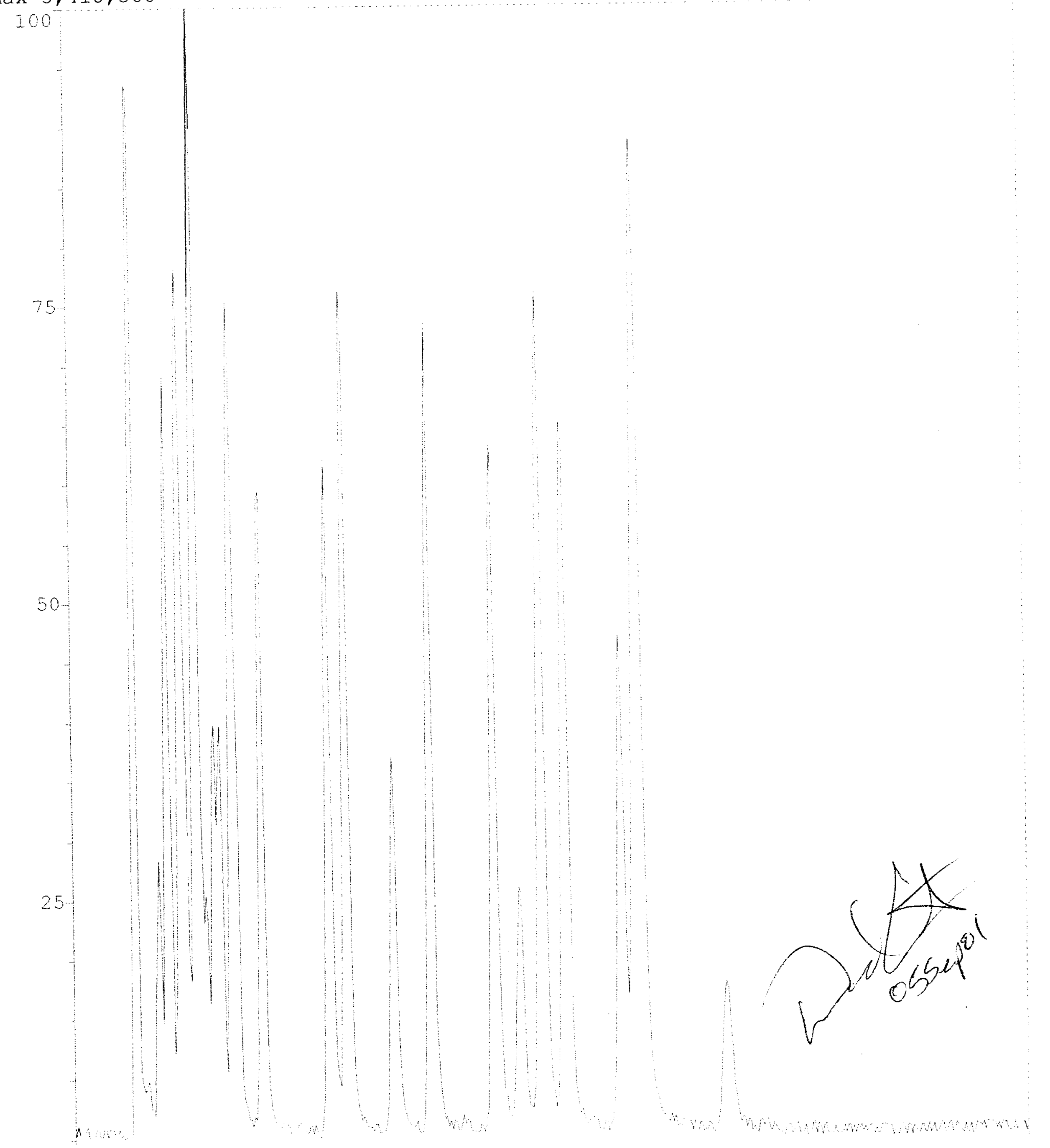

0

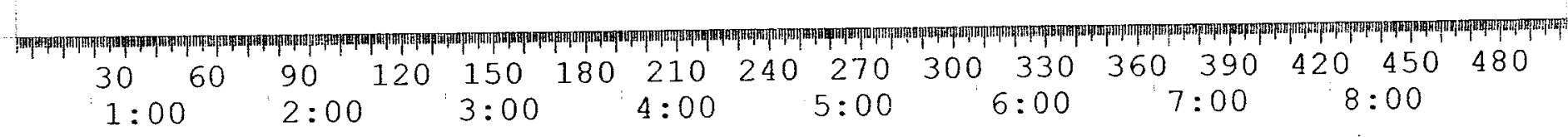


Run: $08 / 31 / 01$ at $23: 49: 33 \quad$ RTime $0: 31.10$ to $22: 16.18$

540 ppmv Jet $A$

TIC $\operatorname{Iax}=5,919,751$

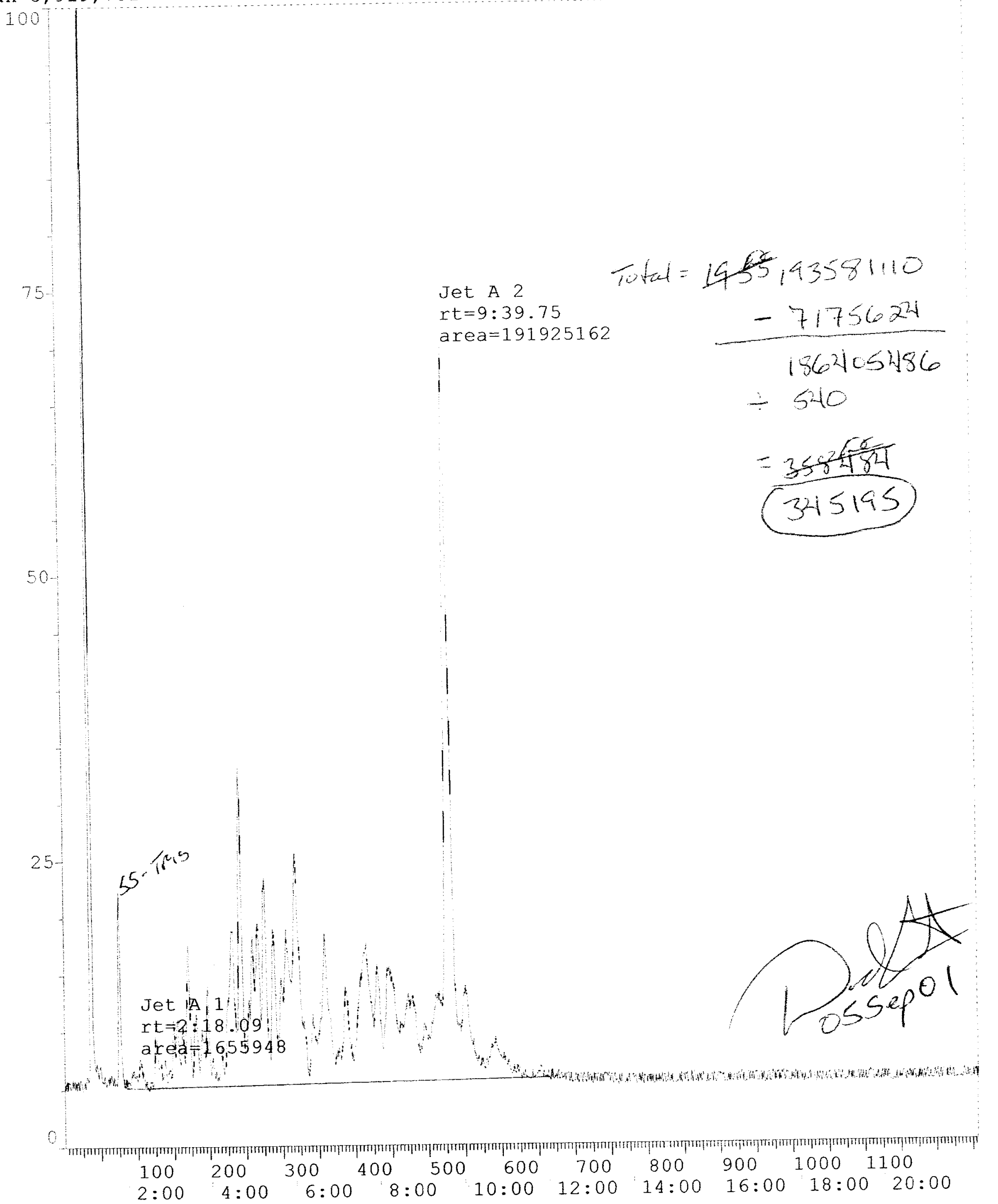


FULL SCAN Quantitation Report

Title: IT NTS Des Rock

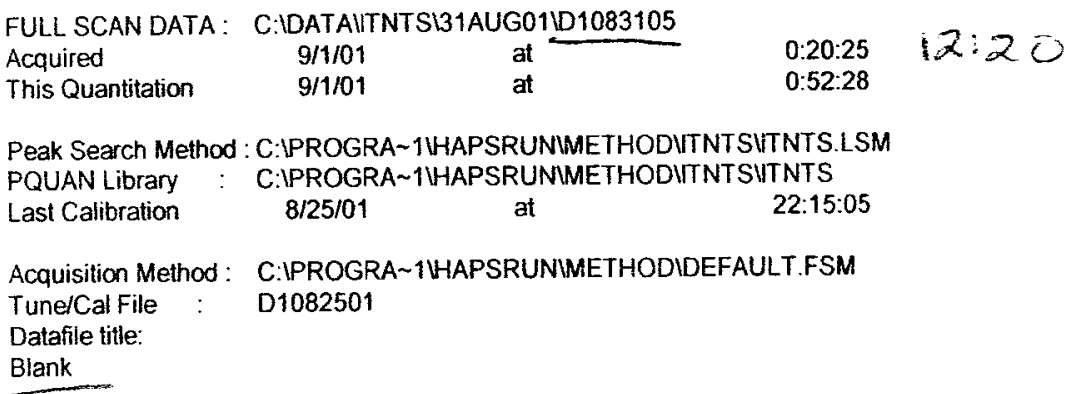

$W=R T+-(0: 20.00 / 2+R T * 0.005)$

Min Fit=0.750; Min Pur=0.200; Min Area $=25000$

Width $=2-40$ scans; Res $=10$ scans; NLM $=1.0$

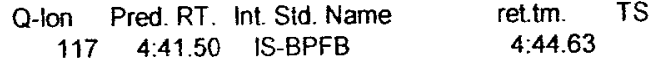

\begin{tabular}{|c|c|c|c|}
\hline Q-Ion & Pred. RT. & Analyte Name & ret.tm. \\
\hline 58 & $1: 22.02$ & Acetone & $1: 20.97$ \\
\hline 101 & $1: 24.11$ & Freon 11 & Not Found \\
\hline 49 & $1: 28.22$ & Methylene Chloride & $1: 31.35$ \\
\hline 61 & $1: 28.22$ & 1,1-Dichloroethene & Not Found \\
\hline 101 & $1: 30.31$ & Freon 113 & Not Found \\
\hline 61 & $1: 34.49$ & trans-1,2-Dichloroet & $1: 43.82$ \\
\hline 73 & $1: 36.57$ & Methyl tert-Butyl Eth & 1:33.44 \\
\hline 63 & $1: 36.57$ & 1.1-Dichloroethane & $1: 35.52$ \\
\hline 72 & $1: 38.61$ & Methyl Ethyl Ketone & Not Found \\
\hline 61 & $1: 43.82$ & cis-1,2-Dichloroethe & $1: 47.99$ \\
\hline 57 & $1: 44.87$ & n-Hexane & $1: 49.04$ \\
\hline 83 & $1: 45.91$ & Chloroform & $1: 44.86$ \\
\hline 213 & $1: 49.04$ & ss-Tris & $1: 50.08$ \\
\hline 62 & $1: 54.20$ & 1,2-Dichloroethane & $1: 49.04$ \\
\hline 97 & $1: 58.38$ & 1,1,1-Trichloroethan & $1: 49.04$ \\
\hline 78 & 2:04.59 & Benzene & $1: 57.33$ \\
\hline 117 & $2: 06.67$ & Carbon Tetrachlorid & $2: 05.62$ \\
\hline 130 & $2: 21.23$ & Trichloroethene & Not Found \\
\hline 97 & $2: 58.63$ & 1,1,2-Trichloroethan & $2: 56.54$ \\
\hline 91 & 3:09.01 & Toluene & $3: 12.14$ \\
\hline 107 & $3: 34.99$ & 1,2-Dibromoethane & $3: 32.90$ \\
\hline 166 & 3.56 .85 & Tetrachloroethene & Not Found \\
\hline 112 & $4: 32.17$ & Chlorobenzene & Not Found \\
\hline 91 & $4: 59.19$ & Ethyl Benzene & $5: 08.53$ \\
\hline 91 & $5: 12.70$ & m,p-Xylene & $5: 11.66$ \\
\hline 104 & $5: 44.89$ & Styrene & $5: 53.18$ \\
\hline 83 & $5: 54.28$ & 1,1,2,2-Tetrachloroe & $5: 48.02$ \\
\hline 91 & $5: 55.27$ & o-Xylene & $5: 53.18$ \\
\hline
\end{tabular}

fit purity MS $0.999 \quad 0.856$

$$
\begin{aligned}
& 1538001 \text { ppmv } 5 \text { Flag } 16.295 \\
& \frac{1538001-1002608}{1538001}=21.3 \% 0 \%
\end{aligned}
$$

fit purity MS area ppmy Flag

$0.972 \quad 0.758$

$0.865 \quad 0.021$

3434

1565

$0.96 \quad 0.133$

$0.95 \quad 0.024$

0.014

394

341

0.958

0.021

$0.877 \quad 0.027$

$0.838 \quad 0.213$

$0.985 \quad 0.942$

$0.952 \quad 0.016$

$\begin{array}{ll}0.605 & 0.044\end{array}$

0.228

0.115

0.014

0.594

0.815

0.725

0.141

0.955

0.884

0.919

0.844

0.894

ppmv
Low area.
Purity too low.
Purity too low.
Purity too low.
Purity too low.
Fit too low.
Purity too low.
Low area.
Purity too low.
Fit too low.
Low area.
Fit too low.
Fit too low.
Low area.
Fit too low.
Low area.
Purity too low.
Purity too low.
Purity too low.
Low area.

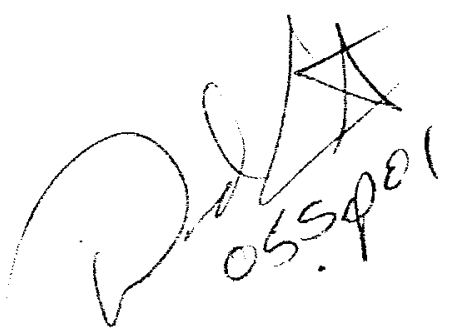


Run: $09 / 01 / 01$ at $00: 20: 25 \quad$ RTime $0: 34.23$ to $14: 09.93$

$\operatorname{Max}=2,965,906$

TIC

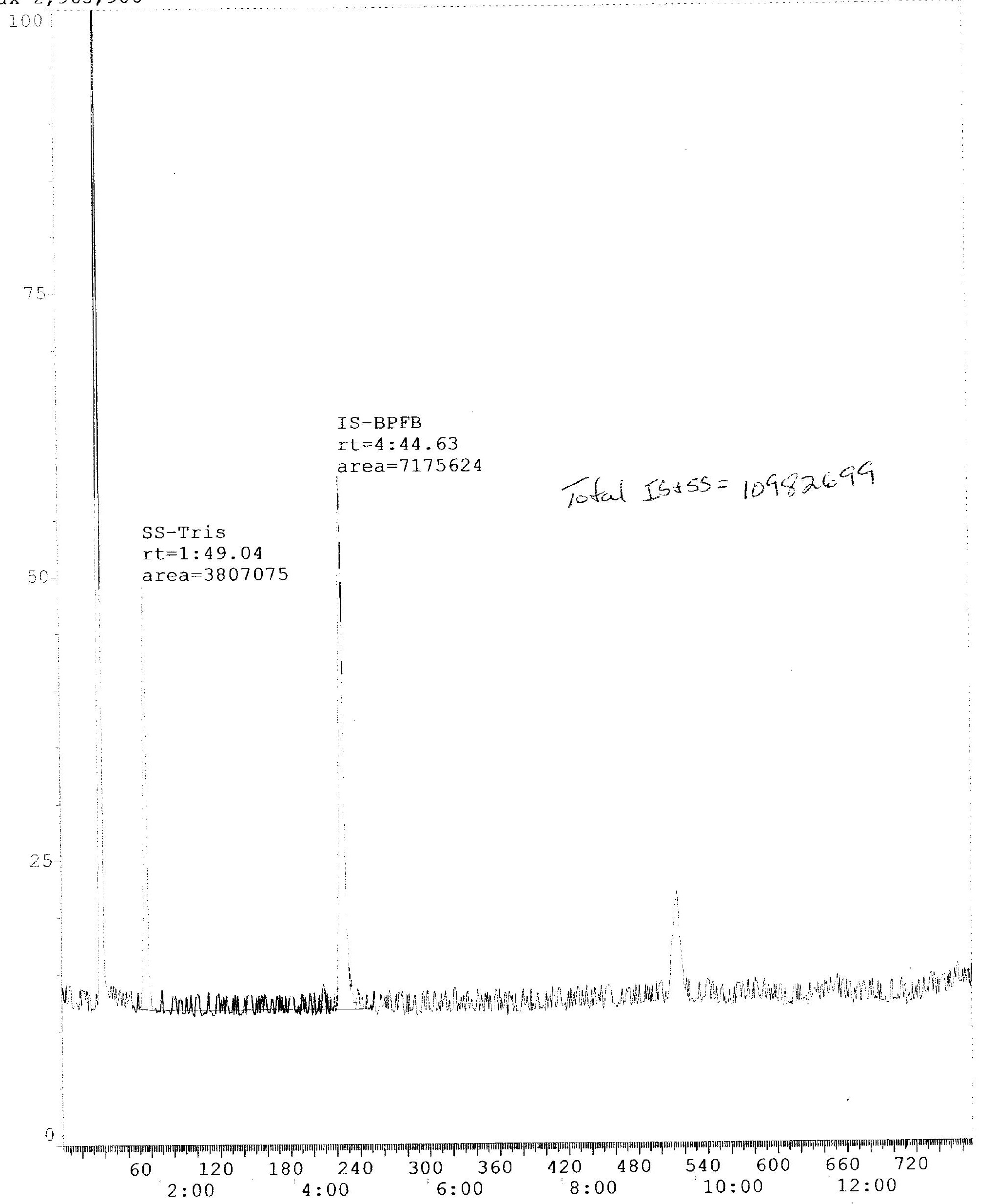


FULL SCAN Quantitation Report

Title: IT NTS Des Rock

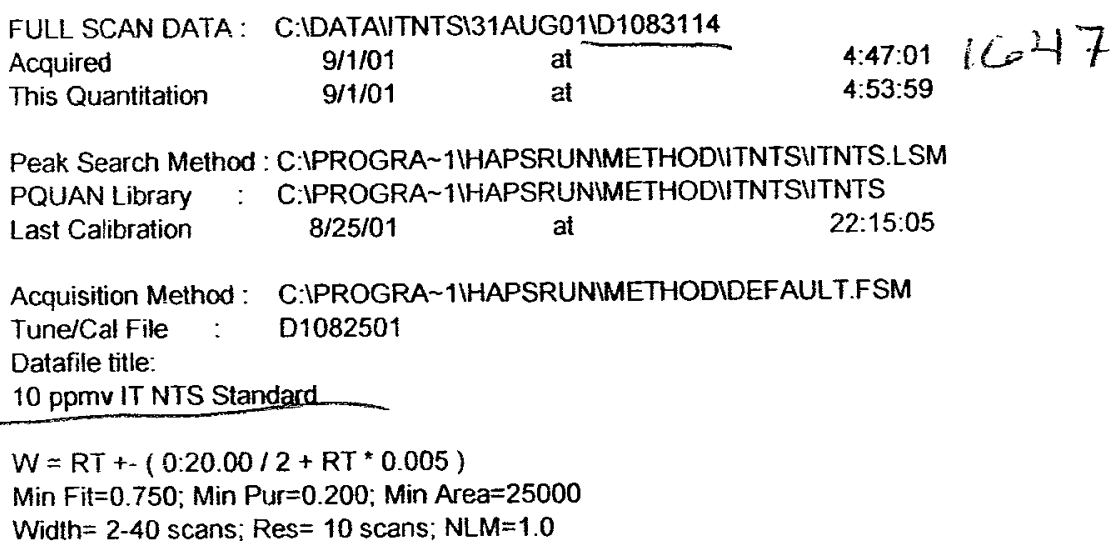

Q-ion Pred. RT. Int. Std. Name ret.tm. TS $117 \quad 4: 41.50 \quad$ IS-BPFB

$\begin{array}{rrll}\text { Q-ion } & \text { Pred. RT. } & \text { Analyte Name } & \text { ret.tm. } \\ 58 & 1: 22.02 & \text { Acetone } & 1: 22.02 \\ 101 & 1: 24.11 & \text { Freon 11 } & 1: 24.10 \\ 49 & 1: 28.22 & \text { Methylene Chloride } & 1: 28.22 \\ 61 & 1: 28.22 & \text { 1,1-Dichloroethene } & 1: 28.22 \\ 101 & 1: 30.31 & \text { Freon 113 } & 1: 30.31 \\ 61 & 1: 34.49 & \text { trans-1,2-Dichloroetl } & 1: 34.48 \\ 73 & 1: 36.57 & \text { Methyl tert-Butyl Eth } & 1: 36.57 \\ 63 & 1: 36.57 & 1,1 \text {-Dichloroethane } & 1: 35.53 \\ 72 & 1: 38.61 & \text { Methyl Ethyl Ketone } & 1: 38.60 \\ 61 & 1: 43.82 & \text { cis-1,2-Dichloroethe } & 1: 43.82 \\ 57 & 1: 44.87 & \text { n-Hexane } & 1: 44.87 \\ 83 & 1: 45.91 & \text { Chloroform } & 1: 45.91 \\ 213 & 1: 49.04 & \text { ss-Tris } & 1: 50.08 \\ 62 & 1: 54.20 & \text { 1,2-Dichloroethane } & 1: 54.20 \\ 97 & 1: 58.38 & 1,1,1 \text {-Trichloroethan } & 1: 58.38 \\ 78 & 2: 04.59 & \text { Benzene } & 2: 04.58 \\ 117 & 2: 06.67 & \text { Carbon Telrachlorioi } & 2: 06.67 \\ 130 & 2: 21.23 & \text { Trichloroethene } & 2: 21.23 \\ 97 & 2: 58.63 & 1,1,2 \text {-Trichloroethan } & 2: 58.63 \\ 91 & 3: 09.01 & \text { Toluene } & 3: 09.01 \\ 107 & 3: 34.99 & \text { 1,2-Dibromoethane } & 3: 34.99 \\ 166 & 3: 56.85 & \text { Tetrachloroethene } & 3: 55.81 \\ 112 & 4: 32.17 & \text { Chlorobenzene } & 4: 32.17 \\ 91 & 4: 59.19 & \text { Ethyl Benzene } & 4: 59.19 \\ 91 & 5: 12.70 & \text { m,p-Xylene } & 5: 12.70 \\ 104 & 5: 44.89 & \text { Styrene } & 5: 43.85 \\ 83 & 5: 54.28 & 1.1,2,2-T e t r a c h l o r o e & 5: 52.20 \\ 91 & 5: 55.27 & \text { o-Xylene } & 5: 54.28\end{array}$
4:46.72

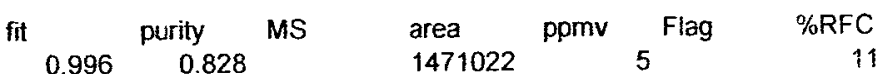
0.828

$1471022 \quad 5$

11.23

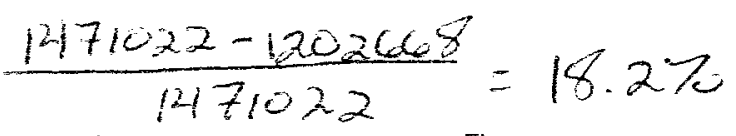

fit

\begin{tabular}{rr}
\multicolumn{2}{c}{ purity } \\
1 & 0.954 \\
1 & 0.976 \\
1 & 0.712 \\
1 & 0.569 \\
0.999 & 0.858 \\
0.997 & 0.94 \\
1 & 0.434 \\
0.998 & 0.708 \\
1 & 0.992 \\
1 & 0.656 \\
1 & 0.777 \\
1 & 0.955 \\
0.995 & 0.941 \\
1 & 0.923 \\
1 & 0.967 \\
1 & 0.939 \\
1 & 0.891 \\
0.998 & 0.906 \\
1 & 0.951 \\
1 & 0.98 \\
1 & 0.99 \\
0.998 & 0.889 \\
0.997 & 0.921 \\
0.999 & 0.968 \\
0.997 & 0.953 \\
1 & 0.802 \\
1 & 0.677 \\
0.998 & 0.861
\end{tabular}

area

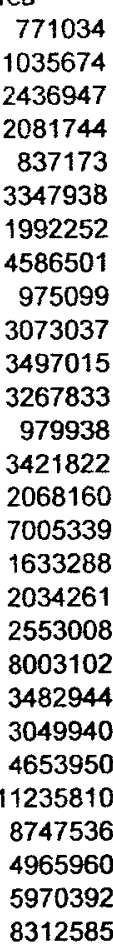

ppmv Flag

$$
\begin{array}{r}
11.49 \\
9 \\
8.85 \\
9.62 \\
9.02 \\
9.72 \\
11.54 \\
9.27 \\
10.91 \\
9.86 \\
9.27 \\
9.33 \\
11.58 \\
9.42 \\
9.09 \\
10.12 \\
7.26 \\
8.2 \\
8.9 \\
10.58 \\
7.8 \\
8.99 \\
7.97 \\
10.76 \\
11.2 \\
9.29 \\
9.7 \\
11.12 \\
\end{array}
$$

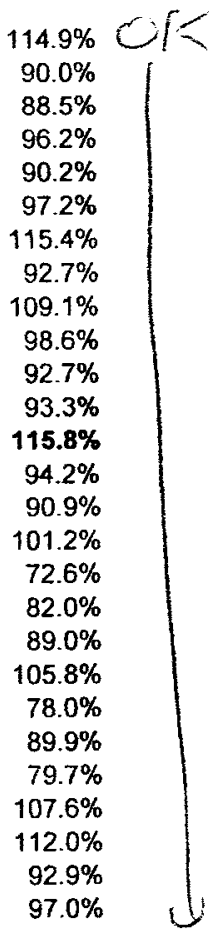

$111.2 \%$

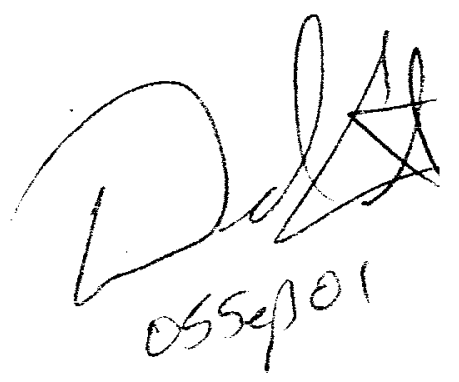




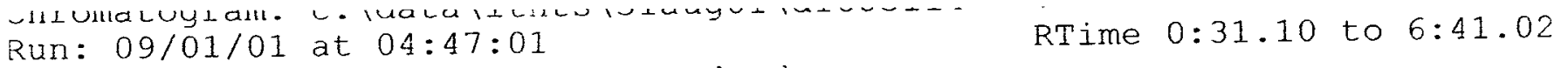

10 ppmv IT NTS standard

TIC

$\operatorname{vax}=7,382,853$ 100

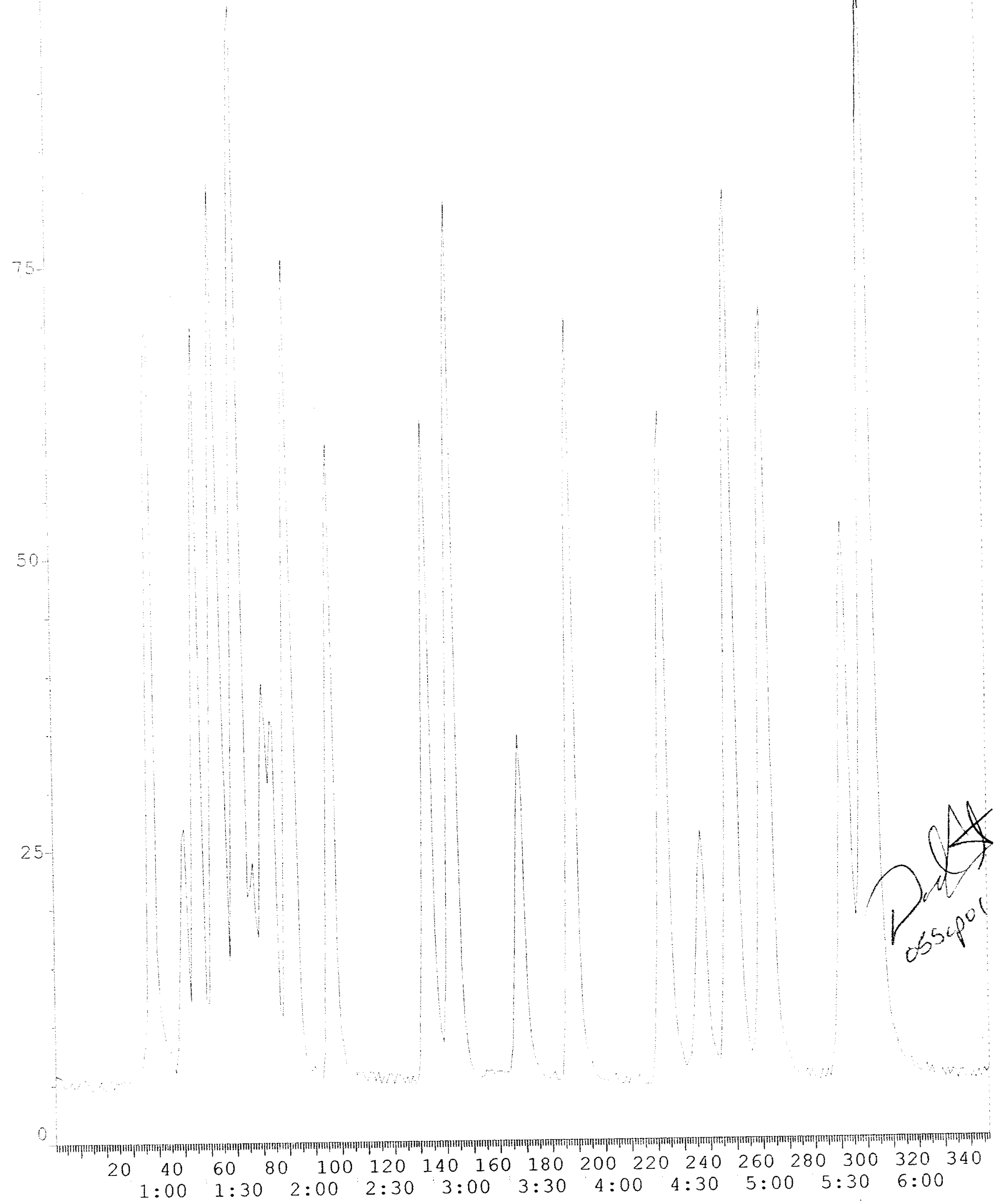


FULL SCAN Calibration Response Table

Title: IT NTS Des Rock

FULL SCAN DATA： C:IDATAITNTSL25AUG01ID1082507

Acquired

$8 / 25 / 01$

at

$19: 47: 37$

Peak Search Method: C:IPROGRA 1IHAPSRUNIMETHODUTNTSITNTS.LSM

PQUAN Library : C:IPROGRA 1 1HAPSRUNIMETHODITNTSITNTS

Last Calibration

$8 / 25 / 01$

at

22:15:05

Acquisition Method : C:IPROGRA 1 1HAPSRUNMETHODIDEFAULT.FSM

Tune/Cal File : D1082501

Datafile title:

100 ppmv ITNTS Standard

$W=R T+-(0: 20.00 / 2+R T * 0.005)$

Min Fit $=0.750$; Min Pur $=0.200$; Min Area $=25000$

Width $=2-40$ scans; Res $=10$ scans; NLM $=1.0$

Calibration curve $=$ Average Response Factor

Internal Standards:

I.S. \#1 - IS-BPFB

Concentration $=5.000 \mathrm{ppmv} ; 6$ calib points

pt.

\begin{tabular}{|c|c|c|c|}
\hline File & Man. & Area & Resp. Factor \\
\hline D108250 & & 1273466 & 254693.2 \\
\hline D1082511 & & 1438514 & 287702.8 \\
\hline D108250: & & 1343914 & 268782.8 \\
\hline D108250 & & 1352189 & 270437.8 \\
\hline D108251 & & 1407477 & 281495.4 \\
\hline D108250: & & 1119440 & 223888 \\
\hline
\end{tabular}

Analytes:

Analyte \#1 -- Acetone

5 calib points

pt.

$\begin{array}{ll}\text { File M M } \\ 1 & \text { D1082506 } \\ 2 & D 1082503 \\ 3 & D 1082504 \\ 4 & D 1082511 \\ 5 & D 1082507\end{array}$

\begin{tabular}{llrll} 
Conc. & Cratio & \multicolumn{1}{c}{ Area } & Aratio & Resp. Factor \\
50 & $1.00 \mathrm{E}+01$ & 2908697 & $2.28 \mathrm{E}+00$ & $2.28 \mathrm{E}-01$ \\
0.5 & $1.00 \mathrm{E}-01$ & 29588 & $2.20 \mathrm{E}-02$ & $2.20 \mathrm{E}-01$ \\
1 & $2.00 \mathrm{E}-01$ & 58499 & $4.33 \mathrm{E}-02$ & $2.16 \mathrm{E}-01$ \\
10 & $2.00 \mathrm{E}+00$ & 588065 & $4.18 \mathrm{E}-01$ & $2.09 \mathrm{E}-01$ \\
100 & $2.00 \mathrm{E}+01$ & 5977847 & $5.34 \mathrm{E}+00$ & $2.67 \mathrm{E}-01$ \\
Average RF $=2.28158 \mathrm{e}-01$ & & \\
Deviation $=10.00353 \%$ &
\end{tabular}


Analyte \#2 -- Freon 11

5 calib points

pt.
File $M$
1 D1082506
2 D1082503
3 D1082504
4 D1082511
$5 \quad$ D1082507

\begin{tabular}{rlrcc} 
Conc. & Cratio & \multicolumn{1}{c}{ Area } & \multicolumn{1}{l}{ Aratio } & Resp. Factor \\
50 & $1.00 \mathrm{E}+01$ & 4953796 & $3.89 \mathrm{E}+00$ & $3.89 \mathrm{E}-01$ \\
0.5 & $1.00 \mathrm{E}-01$ & 52009 & $3.87 \mathrm{E}-02$ & $3.87 \mathrm{E}-01$ \\
1 & $2.00 \mathrm{E}-01$ & 97874 & $7.24 \mathrm{E}-02$ & $3.62 \mathrm{E}-01$ \\
10 & $2.00 \mathrm{E}+00$ & 1076380 & $7.65 \mathrm{E}-01$ & $3.82 \mathrm{E}-01$ \\
100 & $2.00 \mathrm{E}+01$ & 9745774 & $8.71 \mathrm{E}+00$ & $4.35 \mathrm{E}-01$
\end{tabular}

Average RF $=3.91117 \mathrm{e}-01$

Deviation $=6.88760 \%$

Analyte $\# 3-1,1-$ Dichloroethene 6 calib points

pt.

$\begin{array}{ll}\text { File } \\ 1 & \text { D1082506 } \\ 2 & \text { D1082510 } \\ 3 & \text { D1082503 } \\ 4 & \text { D1082504 } \\ 5 & \text { D1082511 } \\ 6 & \text { D1082507 }\end{array}$

Analyte \#4 -- Methylene Chloride 5 calib points

pt.

$\begin{array}{ll}\text { File } \\ 1 & \text { D1082506 } \\ 2 & \text { D1082503 } \\ 3 & \text { D1082504 } \\ 4 & \text { D1082511 } \\ 5 & \text { D1082507 }\end{array}$

Analyte \#5 - Freon 113 5 calib points

$\begin{array}{ll}\text { pt. } & \text { File } \\ 1 & \text { D1082506 } \\ 2 & \text { D1082503 } \\ 3 & \text { D1082504 } \\ 4 & \text { D1082511 } \\ 5 & \text { D1082507 }\end{array}$

$\begin{array}{rlrcc}\text { Conc. } & \text { Cratio } & \text { Area } & \text { Aratio } & \text { Resp. Factor } \\ 50 & 1.00 \mathrm{E}+01 & 9367146 & 7.36 \mathrm{E}+00 & 7.36 \mathrm{E}-01 \\ 0.2 & 4.00 \mathrm{E}-02 & 42371 & 2.95 \mathrm{E}-02 & 7.36 \mathrm{E}-01 \\ 0.5 & 1.00 \mathrm{E}-01 & 99836 & 7.43 \mathrm{E}-02 & 7.43 \mathrm{E}-01 \\ 1 & 2.00 \mathrm{E}-01 & 187770 & 1.39 \mathrm{E}-01 & 6.94 \mathrm{E}-01 \\ 10 & 2.00 \mathrm{E}+00 & 1923700 & 1.37 \mathrm{E}+00 & 6.83 \mathrm{E}-01 \\ 100 & 2.00 \mathrm{E}+01 & 18398520 & 1.64 \mathrm{E}+01 & 8.22 \mathrm{E}-01\end{array}$

Average RF $=7.35714 \mathrm{e}-01$

Deviation $=6.63372 \%$ \begin{tabular}{rrrrr} 
Conc. & Cratio & \multicolumn{1}{c}{ Area } & Aratio & Resp. Factor \\
50 & $1.00 \mathrm{E}+01$ & 3996744 & $3.14 \mathrm{E}+00$ & $3.14 \mathrm{E}-01$ \\
0.5 & $1.00 \mathrm{E}-01$ & 38056 & $2.83 \mathrm{E}-02$ & $2.83 \mathrm{E}-01$ \\
1 & $2.00 \mathrm{E}-01$ & 82772 & $6.12 \mathrm{E}-02$ & $3.06 \mathrm{E}-01$ \\
10 & $2.00 \mathrm{E}+00$ & 901428 & $6.41 \mathrm{E}-01$ & $3.20 \mathrm{E}-01$ \\
100 & $2.00 \mathrm{E}+01$ & 7915303 & $7.07 \mathrm{E}+00$ & $3.54 \mathrm{E}-01$ \\
Average RF $=3.15371 \mathrm{e}-01$ & & \\
Deviation $=8.09343 \%$ & & &
\end{tabular} 
Analyte \#6 -- trans-1,2-Dichloroethene 6 calib points

$\begin{array}{ll}\text { pt. } & \text { File } \\ 1 & \text { D1082506 } \\ 2 & D 1082510 \\ 3 & D 1082503 \\ 4 & D 1082504 \\ 5 & D 1082511 \\ 6 & D 1082507\end{array}$

\begin{tabular}{rrrrr}
\multicolumn{1}{c}{ Conc. } & Cratio & \multicolumn{1}{c}{ Area } & Aratio & Resp. Factor \\
50 & $1.00 \mathrm{E}+01$ & 14413435 & $1.13 \mathrm{E}+01$ & $1.13 \mathrm{E}+00$ \\
0.2 & $4.00 \mathrm{E}-02$ & 80273 & $5.58 \mathrm{E}-02$ & $1.40 \mathrm{E}+00$ \\
0.5 & $1.00 \mathrm{E}-01$ & 146065 & $1.09 \mathrm{E}-01$ & $1.09 \mathrm{E}+00$ \\
1 & $2.00 \mathrm{E}-01$ & 299062 & $2.21 \mathrm{E}-01$ & $1.11 \mathrm{E}+00$ \\
10 & $2.00 \mathrm{E}+00$ & 2935888 & $2.09 \mathrm{E}+00$ & $1.04 \mathrm{E}+00$ \\
100 & $2.00 \mathrm{E}+01$ & 28314796 & $2.53 \mathrm{E}+01$ & $1.26 \mathrm{E}+00$ \\
Average RF $=1.17121 \mathrm{e}+00$ & & \\
Deviation $=11.34238 \%$ & & &
\end{tabular}

Analyte \#7 - 1,1-Dichloroethane 6 calib points
pt.

File
D1082506
2
D1082510
D1082503
4 D1082504
51082511
D1082507
Conc

\begin{tabular}{rlr}
50 & Cratio & \multicolumn{1}{c}{ Area } \\
0.2 & $1.00 \mathrm{E}+01$ & 20140172 \\
0.5 & $1.00 \mathrm{E}-02$ & 99434 \\
1 & $2.00 \mathrm{E}-01$ & 218624 \\
10 & $2.00 \mathrm{E}-01$ & 401876 \\
100 & $2.00 \mathrm{E}+01$ & 4339690 \\
\hline
\end{tabular}
$100 \quad 2.00 E+01 \quad 47721336$
Average RF $=1.68259 \mathrm{e}+00$
Deviation $=13.94837 \%$
Aratio Resp. Factor
$1.58 \mathrm{E}+01 \quad 1.58 \mathrm{E}+00$
6.91E-02 1.73E+00
1.63E-01 1.63E+00
$2.97 \mathrm{E}-01 \quad 1.49 \mathrm{E}+00$
$3.08 \mathrm{E}+00 \quad 1.54 \mathrm{E}+00$

Analyte \#8 - Methyl tert-Butyl Ether 6 calib points
pt.

$\begin{array}{ll}\text { File } \\ 1 & \text { D1082506 } \\ 2 & D 1082510 \\ 3 & D 1082503 \\ 4 & D 1082504 \\ 5 & D 1082511 \\ 6 & \text { D1082507 }\end{array}$
Conc

\begin{tabular}{rlr}
50 & \multicolumn{1}{l}{ Cratio } & \multicolumn{1}{c}{ Area } \\
0.2 & $4.00 \mathrm{E}+01$ & 8446987 \\
0.5 & $1.00 \mathrm{E}-02$ & 26194 \\
1 & $2.00 \mathrm{E}-01$ & 70817 \\
10 & $2.00 \mathrm{E}+00$ & 145034 \\
100 & $2.00 \mathrm{E}+01$ & 17239484
\end{tabular}
Aratio Resp. Factor
6.63E+00 6.63E-01
$1.82 \mathrm{E}-02 \quad 4.55 \mathrm{E}-01$
5.27E-02 5.27E-01
1.07E-01 5.36E-01
$1.14 \mathrm{E}+00 \quad 5.68 \mathrm{E}-01$
$1.54 \mathrm{E}+01 \quad 7.70 \mathrm{E}-01$
Average RF $=5.86699 \mathrm{e}-01$
Deviation $=19.16282 \%$

Analyte \#9 - Methyl Ethyl Ketone 5 calib points

pt.

$\begin{array}{ll}\text { File M } \\ 1 & \text { D1082506 } \\ 2 & \text { D1082503 } \\ 3 & D 1082504 \\ 4 & \text { D1082511 } \\ 5 & \text { D1082507 }\end{array}$

Conc.

$\begin{array}{rlrrr}\text { Conc. } & \text { Cratio } & \text { Area } & \text { Aratio } & \text { Resp. Factor } \\ 50 & 1.00 \mathrm{E}+01 & 4236910 & 3.33 \mathrm{E}+00 & 3.33 \mathrm{E}-01 \\ 0.5 & 1.00 \mathrm{E}-01 & 32244 & 2.40 \mathrm{E}-02 & 2.40 \mathrm{E}-01 \\ 1 & 2.00 \mathrm{E}-01 & 74497 & 5.51 \mathrm{E}-02 & 2.75 \mathrm{E}-01 \\ 10 & 2.00 \mathrm{E}+00 & 807270 & 5.74 \mathrm{E}-01 & 2.87 \mathrm{E}-01 \\ 100 & 2.00 \mathrm{E}+01 & 8614134 & 7.70 \mathrm{E}+00 & 3.85 \mathrm{E}-01\end{array}$

Average RF $=3.03926 \mathrm{e}-01$

Deviation $=18.43802 \%$ 
Analyte \#10 -- cis-1,2-Dichloroethene 6 calib points

pt.

$\begin{array}{ll}\text { File } M \\ 1 & \text { D1082506 } \\ 2 & D 1082510 \\ 3 & D 1082503 \\ 4 & D 1082504 \\ 5 & D 1082511 \\ 6 & D 1082507\end{array}$

\begin{tabular}{rrrrr} 
Conc. & Cratio & \multicolumn{1}{c}{ Area } & Aratio & Resp. Factor \\
50 & $1.00 \mathrm{E}+01$ & 13241462 & $1.04 \mathrm{E}+01$ & $1.04 \mathrm{E}+00$ \\
0.2 & $4.00 \mathrm{E}-02$ & 65049 & $4.52 \mathrm{E}-02$ & $1.13 \mathrm{E}+00$ \\
0.5 & $1.00 \mathrm{E}-01$ & 137503 & $1.02 \mathrm{E}-01$ & $1.02 \mathrm{E}+00$ \\
1 & $2.00 \mathrm{E}-01$ & 283545 & $2.10 \mathrm{E}-01$ & $1.05 \mathrm{E}+00$ \\
10 & $2.00 \mathrm{E}+00$ & 2684092 & $1.91 \mathrm{E}+00$ & $9.54 \mathrm{E}-01$ \\
100 & $2.00 \mathrm{E}+01$ & 25956996 & $2.32 \mathrm{E}+01$ & $1.16 \mathrm{E}+00$ \\
Average RF $=1.05913 \mathrm{e}+00$ & & \\
Deviation $=7.07536 \%$ & & &
\end{tabular}

Analyte \#11 - n-Hexane 6 calib points

pt.

\begin{tabular}{ll}
\multicolumn{1}{l}{ File } \\
1 & D1082506 \\
2 & $D 1082510$ \\
3 & $D 1082503$ \\
4 & D1082504 \\
5 & $D 1082511$ \\
6 & D1082507
\end{tabular}

Analyte \#12 - Chloroform 6 calib points

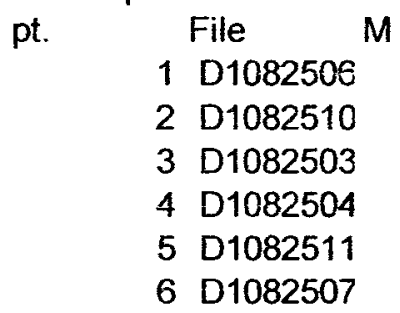

Analyte \#13 - ss-Tris 6 calib points pt.

5 File M

\begin{tabular}{rrrrr} 
Conc. & Cratio & \multicolumn{1}{c}{ Area } & Aratio & Resp. Factor \\
50 & $1.00 \mathrm{E}+01$ & 14913590 & $1.17 \mathrm{E}+01$ & $1.17 \mathrm{E}+00$ \\
0.2 & $4.00 \mathrm{E}-02$ & 101151 & $7.03 \mathrm{E}-02$ & $1.76 \mathrm{E}+00$ \\
0.5 & $1.00 \mathrm{E}-01$ & 167585 & $1.25 \mathrm{E}-01$ & $1.25 \mathrm{E}+00$ \\
1 & $2.00 \mathrm{E}-01$ & 302464 & $2.24 \mathrm{E}-01$ & $1.12 \mathrm{E}+00$ \\
10 & $2.00 \mathrm{E}+00$ & 3058679 & $2.17 \mathrm{E}+00$ & $1.09 \mathrm{E} \div 00$ \\
100 & $2.00 \mathrm{E}+01$ & 29429740 & $2.63 \mathrm{E}+01$ & $1.31 \mathrm{E}+00$ \\
Average RF $=1.28258 \mathrm{e}+00$ & & \\
Deviation $=19.28849 \%$ &
\end{tabular}
Conc. Cratio Area Aratio Resp. Factor $\begin{array}{lllll}50 & 1.00 \mathrm{E}+01 & 14325397 & 1.13 \mathrm{E}+01 & 1.12 \mathrm{E}+00\end{array}$
$0.2 \quad 4.00 \mathrm{E}-02 \quad 75071 \quad 5.22 \mathrm{E}-02 \quad 1.30 \mathrm{E}+00$
$0.5 \quad 1.00 \mathrm{E}-01 \quad 159090 \quad 1.18 \mathrm{E}-01 \quad 1.18 \mathrm{E}+00$
1 2.00E-01 313663 2.32E-01 1.16E+00
$10 \quad 2.00 E+00 \quad 3179825 \quad 2.26 E+00 \quad 1.13 E+00$
$100 \quad 2.00 E+01 \quad 27706494 \quad 2.48 E+01 \quad 1.24 E+00$
Average RF $=1.19005 \mathrm{e}+00$
Deviation $=5.85030 \%$


Analyte \#14 - 1,2-Dichloroethane 6 calib points

\begin{tabular}{llrrrrr} 
pt. & File $M$ & Conc. & \multicolumn{1}{c}{ Cratio } & \multicolumn{1}{c}{ Area } & Aratio & Resp. Factor \\
1 & $\mathrm{D} 1082506$ & 50 & $1.00 \mathrm{E}+01$ & 14872205 & $1.17 \mathrm{E}+01$ & $1.17 \mathrm{E}+00$ \\
2 & $\mathrm{D} 1082510$ & 0.2 & $4.00 \mathrm{E}-02$ & 80226 & $5.58 \mathrm{E}-02$ & $1.39 \mathrm{E}+00$ \\
3 & $\mathrm{D} 1082503$ & 0.5 & $1.00 \mathrm{E}-01$ & 173327 & $1.29 \mathrm{E}-01$ & $1.29 \mathrm{E}+00$ \\
4 & $\mathrm{D} 1082504$ & 1 & $2.00 \mathrm{E}-01$ & 298818 & $2.21 \mathrm{E}-01$ & $1.10 \mathrm{E}+00$ \\
5 & $\mathrm{D} 1082511$ & 10 & $2.00 \mathrm{E}+00$ & 3197327 & $2.27 \mathrm{E}+00$ & $1.14 \mathrm{E}+00$ \\
6 & $\mathrm{D} 1082507$ & 100 & $2.00 \mathrm{E}+01$ & 29368112 & $2.62 \mathrm{E}+01$ & $1.31 \mathrm{E}+00$ \\
\multicolumn{5}{c}{ Average RF $=1.23406 \mathrm{e}+00$} \\
\end{tabular}

Analyte \#15 - 1,1,1-Trichloroethane 6 calib points

$\begin{array}{ll}\text { pt. } & \text { File } \\ 1 & \mathrm{D} 1082506 \\ 2 & \mathrm{D} 1082510 \\ 3 & \mathrm{D} 1082503 \\ 4 & \mathrm{D} 1082504 \\ 5 & \mathrm{D} 1082511 \\ 6 & \mathrm{D} 1082507\end{array}$

\begin{tabular}{rcrcc} 
Conc. & Cratio & \multicolumn{1}{c}{ Area } & Aratio & Resp. Factor \\
50 & $1.00 \mathrm{E}+01$ & 9444688 & $7.42 \mathrm{E}+00$ & $7.42 \mathrm{E}-01$ \\
0.2 & $4.00 \mathrm{E}-02$ & 47176 & $3.28 \mathrm{E}-02$ & $8.20 \mathrm{E}-01$ \\
0.5 & $1.00 \mathrm{E}-01$ & 102414 & $7.62 \mathrm{E}-02$ & $7.62 \mathrm{E}-01$ \\
1 & $2.00 \mathrm{E}-01$ & 201249 & $1.49 \mathrm{E}-01$ & $7.44 \mathrm{E}-01$ \\
10 & $2.00 \mathrm{E}+00$ & 2073734 & $1.47 \mathrm{E}+00$ & $7.37 \mathrm{E}-01$ \\
100 & $2.00 \mathrm{E}+01$ & 18676788 & $1.67 \mathrm{E}+01$ & $8.34 \mathrm{E}-01$
\end{tabular}

Average RF $=7.73105 \mathrm{e}-01$

Deviation $=5.54716 \%$

Analyte \#16 - Benzene 6 calib points

$\begin{array}{ll}\text { pt. } & \text { File } \\ 1 & \text { D1082506 } \\ 2 & \text { D1082510 } \\ 3 & \text { D1082503 } \\ 4 & \text { D1082504 } \\ 5 & \text { D1082511 } \\ 6 & \text { D1082507 }\end{array}$

\begin{tabular}{rcrcc} 
Conc. & Cratio & \multicolumn{1}{c}{ Area } & \multicolumn{1}{l}{ Aratio } & Resp. Factor \\
50 & $1.00 \mathrm{E}+01$ & 30334992 & $2.38 \mathrm{E}+01$ & $2.38 \mathrm{E}+00$ \\
0.2 & $4.00 \mathrm{E}-02$ & 132362 & $9.20 \mathrm{E}-02$ & $2.30 \mathrm{E}+00$ \\
0.5 & $1.00 \mathrm{E}-01$ & 315373 & $2.35 \mathrm{E}-01$ & $2.35 \mathrm{E}+00$ \\
1 & $2.00 \mathrm{E}-01$ & 601891 & $4.45 \mathrm{E}-01$ & $2.23 \mathrm{E}+00$ \\
10 & $2.00 \mathrm{E}+00$ & 6393023 & $4.54 \mathrm{E}+00$ & $2.27 \mathrm{E}+00$ \\
100 & $2.00 \mathrm{E}+01$ & 57902944 & $5.17 \mathrm{E}+01$ & $2.59 \mathrm{E}+00$
\end{tabular}

Average RF $=2.35201 e+00$

Deviation $=5.41129 \%$

Analyte \#17 -- Carbon Tetrachloride 6 calib points

pt.

$\begin{array}{ll}\text { File } \\ 1 & \mathrm{D} 1082506 \\ 2 & \mathrm{D} 1082510 \\ 3 & \mathrm{D} 1082503 \\ 4 & \mathrm{D} 1082504 \\ 5 & \mathrm{D} 1082511 \\ 6 & \mathrm{D} 1082507\end{array}$
Conc. Cratio
$50 \quad 1.00 E+01$
0.2 4.00E-02
Area 9362074
$0.5 \quad 1.00 \mathrm{E}-01$ 43890
1 2.00E-01 204899
$10 \quad 2.00 E+00 \quad 2094212$
$100 \quad 2.00 E+01 \quad 18381732$

Aratio Resp. Factor

$7.35 \mathrm{E}+00$

7.35E-01

3.05E-02 7.63E-01

7.71E-02 7.71E-01

1.52E-01 7.58E-01

$1.49 \mathrm{E}+00$

7.44E-01

$1.64 \mathrm{E}+01$

8.21E-01

Average RF $=7.65188 \mathrm{e}-01$

Deviation $=3.94711 \%$ 
Analyte \#18 - Trichloroethene 6 calib points

pt.

$\begin{array}{ll}\text { File } \\ 1 & \text { D1082506 } \\ 2 & D 1082510 \\ 3 & D 1082503 \\ 4 & D 1082504 \\ 5 & D 1082511 \\ 6 & \text { D1082507 }\end{array}$
Conc. Cratio Area $50 \quad 1.00 \mathrm{E}+01 \quad 10479930$
$0.2 \quad 4.00 E-02 \quad 53231$
$0.5 \quad 1.00 \mathrm{E}-01 \quad 107966$
$1 \quad 2.00 \mathrm{E}-01 \quad 219196$
$10 \quad 2.00 E+00 \quad 2192113$
$100 \quad 2.00 E+0120530740$
Average RF $=8.42948 \mathrm{e}-01$
Deviation $=7.38482 \%$

Analyte \#19-1,1,2-Trichloroethane

6 calib points

pt.

$\begin{array}{ll}\text { File } \\ 1 & \text { D1082506 } \\ 2 & \text { D1082510 } \\ 3 & \text { D1082503 } \\ 4 & \text { D1082504 } \\ 5 & \text { D1082511 } \\ 6 & \text { D1082507 }\end{array}$
Conc. Cratio Area $50 \quad 1.00 E+0111960467$ $0.2 \quad 4.00 \mathrm{E}-02 \quad 59450$ $0.5 \quad 1.00 \mathrm{E}-01 \quad 129291$ 1 2.00E-01 256879 $10 \quad 2.00 \mathrm{E}+00 \quad 2563117$ $100 \quad 2.00 E+0123661272$ Average RF $=9.75279 \mathrm{e}-01$ Deviation $=5.85767 \%$

Analyte \#20 - Toluene 6 calib points

pt.

$\begin{array}{ll}\text { File } \\ 1 & \text { D1082506 } \\ 2 & D 1082510 \\ 3 & D 1082503 \\ 4 & D 1082504 \\ 5 & D 1082511 \\ 6 & D 1082507\end{array}$

Conc.

\begin{tabular}{rlr}
50 & \multicolumn{1}{l}{ Cratio } & \multicolumn{1}{c}{ Area } \\
0.2 & $4.00 \mathrm{E}+01$ & 35207904 \\
0.5 & $1.00 \mathrm{E}-02$ & 142340 \\
1 & $2.00 \mathrm{E}-01$ & 319126 \\
10 & $2.00 \mathrm{E}+00$ & 6963588 \\
100 & $2.00 \mathrm{E}+01$ & 68367536
\end{tabular}

Aratio Resp. Factor $9.39 \mathrm{E}+00$ Resp. Factor 4.13E-02 1.03E+00 9.62E-02 9.62E-01 1.90E-01 9.50E-01 1.82E+00 9.11E-01 $2.11 E+01 \quad 1.06 E+00$

8.23E+00 8.23E-01 3.70E-02 9.25E-01 8.03E-02 8.03E-01 1.62E-01 8.11E-01 $1.56 \mathrm{E}+00 \quad 7.79 \mathrm{E}-01$ $1.83 E+01 \quad 9.17 E-01$

$100 \quad 2.00 E+0168367536$

Average $\mathrm{RF}=2.57137 \mathrm{e}+00$

Deviation $=11.11201 \%$

Analyte \#21 - 1,2-Dibromoethane 6 calib points
pt.

$\begin{array}{ll}\text { File } M \\ 1 & \text { D1082506 } \\ 2 & D 1082510 \\ 3 & D 1082503 \\ 4 & D 1082504 \\ 5 & D 1082511 \\ 6 & D 1082507\end{array}$
1 File 1082506
2 D1082510
3 D1082503
4 D1082504
$\begin{array}{ll}6 & \mathrm{D} 1082507\end{array}$

Conc

Area 2.77E+01 $2.76 \mathrm{E}+00$ 9.90E-02 2.47E+00 2.38E-01 2.37E+00 4.58E-01 2.29E+00 4.95E+00 2.47E+00 $6.11 E+01 \quad 3.05 E+00$
$50 \quad 1.00 \mathrm{E}+01 \quad 18735926$
$0.2 \quad 4.00 \mathrm{E}-02 \quad 100427$
$0.5 \quad 1.00 \mathrm{E}-01 \quad 196608$
$1 \quad 2.00 \mathrm{E}-01 \quad 381806$
$10 \quad 2.00 E+00 \quad 3789582$
$100 \quad 2.00 \mathrm{E}+0137352212$
Average RF $=1.51765 \mathrm{e}+00$
Deviation $=10.21956 \%$

Aratio Resp. Factor

$1.47 E+01 \quad 1.47 E+00$

6.98E-02 1.75E+00

1.46E-01 1.46E+00

2.82E-01 1.41E+00

$2.69 \mathrm{E}+00 \quad 1.35 \mathrm{E}+00$ 
Analyte \#22 -- Tetrachloroethene 6 calib points

pt.

$\begin{array}{ll}\text { File } \\ 1 & \text { D1082506 } \\ 2 & \text { D1082510 } \\ 3 & \text { D1082503 } \\ 4 & \text { D1082504 } \\ 5 & \text { D1082511 } \\ 6 & \text { D1082507 }\end{array}$

Anaiyte \#23 - Chlorobenzene 6 calib points

pt.

$\begin{array}{ll}\text { File } \\ 1 & \text { D1082506 } \\ 2 & D 1082510 \\ 3 & D 1082503 \\ 4 & D 1082504 \\ 5 & D 1082511 \\ 6 & \text { D1082507 }\end{array}$

Analyte \#24 - Ethyl Benzene 6 calib points

pt.

$\begin{array}{ll}1 & \text { File } 1082506 \\ 2 & \text { D1082510 } \\ 3 & \text { D1082503 } \\ 4 & \text { D1082504 } \\ 5 & \text { D1082511 } \\ 6 & \text { D1082507 }\end{array}$

Analyte \#25-m,p-Xylene 6 calib points

$\begin{array}{ll}\text { pt. } & \text { File } \\ 1 & \text { D1082506 } \\ 2 & \text { D1082510 } \\ 3 & \text { D1082503 } \\ 4 & \text { D1082504 } \\ 5 & \text { D1082511 } \\ 6 & \text { D1082507 }\end{array}$

$$
\begin{array}{rlrll}
\text { Conc. } & \text { Cratio } & \multicolumn{1}{c}{\text { Area }} & \text { Aratio } & \text { Resp. Factor } \\
50 & 1.00 E+01 & 14592016 & 1.15 \mathrm{E}+01 & 1.15 \mathrm{E}+00 \\
0.2 & 4.00 \mathrm{E}-02 & 67125 & 4.67 \mathrm{E}-02 & 1.17 \mathrm{E}+00 \\
0.5 & 1.00 \mathrm{E}-01 & 153224 & 1.14 \mathrm{E}-01 & 1.14 \mathrm{E}+00 \\
1 & 2.00 \mathrm{E}-01 & 302996 & 2.24 \mathrm{E}-01 & 1.12 \mathrm{E}+00 \\
10 & 2.00 \mathrm{E}+00 & 3020372 & 2.15 \mathrm{E}+00 & 1.07 \mathrm{E}+00 \\
100 & 2.00 \mathrm{E}+01 & 28574788 & 2.55 \mathrm{E}+01 & 1.28 \mathrm{E}+00
\end{array}
$$

Average RF $=1.15370 \mathrm{e}+00$

Deviation $=5.88742 \%$

\begin{tabular}{rlrll} 
Conc. & Cratio & Area & \multicolumn{1}{l}{ Aratio } & Resp. Factor \\
50 & $1.00 \mathrm{E}+01$ & 26244732 & $2.06 \mathrm{E}+01$ & $2.06 \mathrm{E}+00$ \\
0.2 & $4.00 \mathrm{E}-02$ & 115671 & $8.04 \mathrm{E}-02$ & $2.01 \mathrm{E}+00$ \\
0.5 & $1.00 \mathrm{E}-01$ & 254627 & $1.90 \mathrm{E}-01$ & $1.89 \mathrm{E}+00$ \\
1 & $2.00 \mathrm{E}-01$ & 482902 & $3.57 \mathrm{E}-01$ & $1.79 \mathrm{E}+00$ \\
10 & $2.00 \mathrm{E}+00$ & 5065580 & $3.60 \mathrm{E}+00$ & $1.80 \mathrm{E}+00$ \\
100 & $2.00 \mathrm{E}+01$ & 52884064 & $4.72 \mathrm{E}+01$ & $2.36 \mathrm{E}+00$
\end{tabular}

Average RF $=1.98551 \mathrm{e}+00$

Deviation $=10.82299 \%$

$\begin{array}{rlrrr}\text { Conc. } & \text { Cratio } & \text { Area } & \text { Aratio } & \text { Resp. Factor } \\ 50 & 1.00 \mathrm{E}+01 & 51132688 & 4.02 \mathrm{E}+01 & 4.02 \mathrm{E}+00 \\ 0.2 & 4.00 \mathrm{E}-02 & 181313 & 1.26 \mathrm{E}-01 & 3.15 \mathrm{E}+00 \\ 0.5 & 1.00 \mathrm{E}-01 & 405547 & 3.02 \mathrm{E}-01 & 3.02 \mathrm{E}+00 \\ 1 & 2.00 \mathrm{E}-01 & 850734 & 6.29 \mathrm{E}-01 & 3.15 \mathrm{E}+00 \\ 10 & 2.00 \mathrm{E}+00 & 9781989 & 6.95 \mathrm{E}+00 & 3.48 \mathrm{E}+00 \\ 100 & 2.00 \mathrm{E}+01 & 1 \mathrm{E}+08 & 8.97 \mathrm{E}+01 & 4.49 \mathrm{E}+00 \\ \text { Average RF }=3.54850 \mathrm{e}+00 & & \\ \text { Deviation }=16.45456 \% & \end{array}$

Conc. Cratio Area Aratio Resp. Factor

$\begin{array}{rrrrr}50 & 1.00 E+01 & 39738176 & 3.12 \mathrm{E}+01 & 3.12 \mathrm{E}+00 \\ 0.2 & 4.00 \mathrm{E}-02 & 138863 & 9.65 \mathrm{E}-02 & 2.41 \mathrm{E}+00 \\ 0.5 & 1.00 \mathrm{E}-01 & 272286 & 2.03 \mathrm{E}-01 & 2.03 \mathrm{E}+00 \\ 1 & 2.00 \mathrm{E}-01 & 611337 & 4.52 \mathrm{E}-01 & 2.26 \mathrm{E}+00 \\ 10 & 2.00 \mathrm{E}+00 & 7293998 & 5.18 \mathrm{E}+00 & 2.59 \mathrm{E}+00 \\ 100 & 2.00 \mathrm{E}+01 & 78816448 & 7.04 \mathrm{E}+01 & 3.52 \mathrm{E}+00\end{array}$

Average RF $=2.65532 \mathrm{e}+00$

Deviation $=21.16455 \%$ 
Analyte \#26 - Styrene

6 calib points

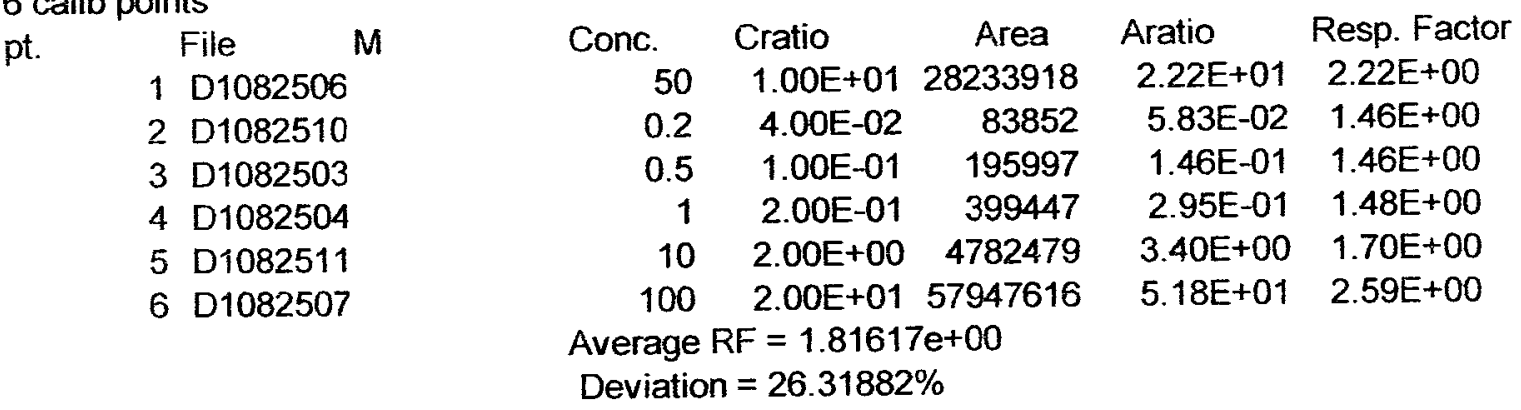

Analyte \#27 - 1,1,2,2-Tetrachloroethane

6 calib points

$\begin{array}{lll}\text { pt. } & \text { File } \\ & 1 & \text { D1082506 } \\ 2 & D 1082510 \\ 3 & D 1082503 \\ 4 & D 1082504 \\ 5 & D 1082511 \\ 6 & D 1082507\end{array}$

Analyte \$28 - o-Xylene 6 calib points

pt.

$\begin{array}{ll}\text { File } M \\ 1 & \text { D1082506 } \\ 2 & D 1082510 \\ 3 & D 1082503 \\ 4 & D 1082504 \\ 5 & D 1082511 \\ 6 & \text { D1082507 }\end{array}$

\begin{tabular}{rlrrr} 
Conc. & \multicolumn{1}{l}{ Cratio } & \multicolumn{1}{c}{ Area } & \multicolumn{1}{l}{ Aratio } & Resp. Factor \\
50 & $1.00 \mathrm{E}+01$ & 26557174 & $2.09 \mathrm{E}+01$ & $2.09 \mathrm{E}+00$ \\
0.2 & $4.00 \mathrm{E}-02$ & 124043 & $8.62 \mathrm{E}-02$ & $2.16 \mathrm{E}+00$ \\
0.5 & $1.00 \mathrm{E}-01$ & 275777 & $2.05 \mathrm{E}-01$ & $2.05 \mathrm{E}+00$ \\
1 & $2.00 \mathrm{E}-01$ & 550906 & $4.07 \mathrm{E}-01$ & $2.04 \mathrm{E}+00$ \\
10 & $2.00 \mathrm{E}+00$ & 5157441 & $3.66 \mathrm{E}+00$ & $1.83 \mathrm{E}+00$ \\
100 & $2.00 \mathrm{E}+01$ & 53514168 & $4.78 \mathrm{E}+01$ & $2.39 \mathrm{E}+00$ \\
Average RF $=2.09211 \mathrm{e}+00$ & &
\end{tabular}
Conc. Cratio Area Aratio Resp. Factor $50 \quad 1.00 E+01 \quad 37801352 \quad 2.97 E+01 \quad 2.97 E+00$
$0.2 \quad 4.00 \mathrm{E}-02 \quad 118191 \quad 8.22 \mathrm{E}-02 \quad 2.05 \mathrm{E}+00$
$\begin{array}{lllll}0.5 & 1.00 \mathrm{E}-01 & 298650 & 2.22 \mathrm{E}-01 & 2.22 \mathrm{E}+00\end{array}$
$1 \quad 2.00 E-01 \quad 605487 \quad 4.48 E-01 \quad 2.24 E+00$
$10 \quad 2.00 E+00 \quad 6937095 \quad 4.93 E+00 \quad 2.46 E+00$
$100 \quad 2.00 E+01 \quad 73739176 \quad 6.59 E \div 01 \quad 3.29 E+00$




\section{Performance Analytical Inc.}

Air Quality Laboratory

A Division of Columbia Analvital Sentees. In

An Emploree Owned Company

\section{RESULTS OF ANALYSIS}

PAGE 1 OF 1

\section{Client : Field Portable Analytical Inc.}

Client Sample ID : DRAO \#1

PAI Sample ID : P2102120-001

PAI Project ID : P2102120

Test Code : Modified EPA TO-13A

Date Sampled : 8/31/01

Instrument : HP5890II/MSD

Date Received : 9/14/01

Analyst : Nelyn Quitoviera

Date Extracted : 9/24/01

Matrix : PUF/XAD-2

Date Analyzed : 9/27/01

Final Volume: $\quad 1.0 \mathrm{ml}$

Volume Analyzed : $\quad$ NA Liter(s)

D.F. $=1.00$

D.F. $=5.00$

\begin{tabular}{|c|c|c|c|c|c|c|c|}
\hline CAS \# & COMPOUND & $\begin{array}{l}\text { RESULT } \\
\mu \mathrm{g} / \text { Sample }\end{array}$ & $\begin{array}{c}\text { RESULT } \\
\mu \mathrm{g} / \mathrm{m} 3\end{array}$ & $\begin{array}{c}\text { REPORTING } \\
\text { LIMIT } \\
\mu \mathrm{g} / \mathrm{m} 3\end{array}$ & $\begin{array}{c}\text { RESULT } \\
\text { ppbV }\end{array}$ & $\begin{array}{c}\text { REPORTING } \\
\text { LIMIT } \\
\text { ppbV }\end{array}$ & $\begin{array}{c}\text { Data } \\
\text { Qualifier }\end{array}$ \\
\hline 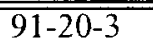 & Naphthalene & 23 & $\overline{\mathrm{NA}}$ & $\overline{\mathrm{NA}}$ & $\overline{\mathrm{NA}}$ & $\overline{\mathrm{NA}}$ & $\overline{\mathrm{EH}}$ \\
\hline $208-96-8$ & Acenaphthalene & $<0.50$ & $\mathrm{NA}$ & NA & NA & NA & EH \\
\hline $83-32-9$ & Acenaphthene & $<0.50$ & $\mathrm{NA}$ & $\overline{\mathrm{NA}}$ & $\overline{\mathrm{NA}}$ & NA & $\mathrm{EH}$ \\
\hline $86-73-7$ & Fluorene & $<0.50$ & $\mathrm{NA}$ & NA & NA & NA & $\overline{\mathrm{EH}}$ \\
\hline $85-01-8$ & Phenanthrene & $<0.50$ & NA & NA & NA & $\overline{\mathrm{NA}}$ & $\overline{\mathrm{EH}}$ \\
\hline $120-12-7$ & Anthracene & $<0.50$ & $\mathrm{NA}$ & $\overline{N A}$ & NA & NA & EH \\
\hline $206-44-0$ & Fluoranthene & $<0.50$ & $\mathrm{NA}$ & $\overline{\mathrm{NA}}$ & NA & $\overline{\mathrm{NA}}$ & $\overline{\mathrm{EH}}$ \\
\hline $129-00-0$ & Pyrene & $<0.50$ & $\mathrm{NA}$ & $\mathrm{NA}$ & NA & $\overline{\mathrm{NA}}$ & $\overline{\mathrm{EH}}$ \\
\hline $56-55-3$ & Benzo(a)anthracene & $<0.50$ & $\mathrm{NA}$ & NA & NA & NA & EH \\
\hline $218-01-9$ & Chrysene & $<0.50$ & $\mathrm{NA}$ & $\mathrm{NA}$ & NA & NA & $\overline{\mathrm{EH}}$ \\
\hline $205-99-2$ & Benzo(b)fluoranthene & $<0.50$ & NA & NA & NA & NA & $\mathrm{EH}$ \\
\hline $207-08-9$ & Benzo(k)fluoranthene & $<0.50$ & $\mathrm{NA}$ & $\overline{\mathrm{NA}}$ & $\mathrm{NA}$ & NA & $\overline{E H}$ \\
\hline $50-32-8$ & Benzo(a)pyrene & $<0.50$ & $\mathrm{NA}$ & NA & $\mathrm{NA}$ & NA & $\overline{\mathrm{EH}}$ \\
\hline $193-39-5$ & Indeno(1,2,3-cd)pyrene & $<0.50$ & NA & NA & NA & NA & EH \\
\hline $53-70-3$ & Dibenzo(a,h)anthracene & $<0.50$ & $\mathrm{NA}$ & $\mathrm{NA}$ & $\mathrm{NA}$ & NA & EH \\
\hline 191-24-2 & Benzo(g,h,i)perylene & $<0.50$ & $\mathrm{NA}$ & $\mathrm{NA}$ & $\mathrm{NA}$ & NA & $\mathrm{EH}$ \\
\hline
\end{tabular}

$\mathrm{ND}=$ Compound was analyzed for, but not detected above the laboratory reporting limit.

$\mathrm{NA}=$ Not Applicable

$\mathrm{EH}=$ Sample extracted outside of extraction hold time, sample results should be evaluated. 
Performance Analytical Inc.

Air Quality Laboratory

A Division of Columbia Analyeicul Serrices lin

an Emplovere Onned Compun

\section{RESULTS OF ANALYSIS}

PAGE 1 OF 1

\section{Client : Field Portable Analytical Inc.}

Client Sample ID : DRAO \#2

PAI Sample ID : P2102120-002

PAI Project ID : P2102120

Test Code : Modified EPA TO-13A

Instrument : HP5890II/MSD

Analyst : Nelyn Quitoviera

Matrix : PUF/XAD-2
Date Sampled : 8/31/01

Date Received : 9/14/01

Date Extracted : 9/24/01

Date Analyzed : 9/27/01

Final Volume: $\quad 1.0 \mathrm{ml}$

Volume Analyzed : $\quad$ NA Liter(s)

D.F. $=1.00$

D.F. $=10.0$

\begin{tabular}{|c|c|c|c|c|c|c|c|}
\hline CAS \# & COMPOUND & $\begin{array}{c}\text { RESULT } \\
\mu \mathrm{g} / \text { Sample }\end{array}$ & $\begin{array}{c}\text { RESULT } \\
\mu \mathrm{g} / \mathrm{m} 3\end{array}$ & $\begin{array}{c}\text { REPORTING } \\
\text { LIMIT } \\
\mu \mathrm{g} / \mathrm{m} 3 \\
\end{array}$ & $\begin{array}{c}\text { RESULT } \\
\text { ppbV }\end{array}$ & $\begin{array}{c}\text { REPORTING } \\
\text { LIMIT } \\
\text { ppbV }\end{array}$ & $\begin{array}{c}\text { Data } \\
\text { Qualifier }\end{array}$ \\
\hline $91-20-3$ & Naphthalene & $\overline{\overline{44}}$ & $\overline{\mathrm{NA}}$ & $\overline{\mathrm{NA}}$ & $\overline{\overline{\mathrm{NA}}}$ & $\overline{\mathrm{NA}}$ & $\mathrm{EH}$ \\
\hline $208-96-8$ & Acenaphthalene & $<0.50$ & $\mathrm{NA}$ & $\overline{\mathrm{NA}}$ & $\overline{\mathrm{NA}}$ & $\mathrm{NA}$ & EH \\
\hline $83-32-9$ & Acenaphthene & $<0.50$ & $\mathrm{NA}$ & NA & $\mathrm{NA}$ & $\mathrm{NA}$ & $\mathrm{EH}$ \\
\hline $86-73-7$ & Fluorene & $<0.50$ & $\mathrm{NA}$ & $\overline{\mathrm{NA}}$ & $\overline{\mathrm{NA}}$ & $\mathrm{NA}$ & $\overline{\mathrm{EH}}$ \\
\hline $85-01-8$ & Phenanthrene & $<0.50$ & $\mathrm{NA}$ & NA & $\mathrm{NA}$ & NA & $\mathrm{EH}$ \\
\hline $120-12-7$ & Anthracene & $<0.50$ & $\mathrm{NA}$ & $\mathrm{NA}$ & $\overline{\mathrm{NA}}$ & NA & $\mathrm{EH}$ \\
\hline $206-44-0$ & Fluoranthene & $<0.50$ & $\mathrm{NA}$ & $\mathrm{NA}$ & $\mathrm{NA}$ & $\mathrm{NA}$ & EH \\
\hline $129-00-0$ & Pyrene & $<0.50$ & $\mathrm{NA}$ & $\overline{\mathrm{NA}}$ & $\mathrm{NA}$ & $\mathrm{NA}$ & $\overline{\mathrm{EH}}$ \\
\hline $56-55-3$ & Benzo(a)anthracene & $<0.50$ & $\overline{N A}$ & $\mathrm{NA}$ & $\overline{\mathrm{NA}}$ & $\mathrm{NA}$ & $\overline{\mathrm{EH}}$ \\
\hline $218-01-9$ & Chrysene & $<0.50$ & $\mathrm{NA}$ & NA & NA & $\mathrm{NA}$ & $\mathrm{EH}$ \\
\hline $205-99-2$ & Benzo(b)fluoranthene & $<0.50$ & $\overline{\mathrm{NA}}$ & NA & $\mathrm{NA}$ & $\mathrm{NA}$ & $\mathrm{EH}$ \\
\hline $207-08-9$ & Benzo(k)fluoranthene & $<0.50$ & $\mathrm{NA}$ & NA & $\mathrm{NA}$ & $\mathrm{NA}$ & $\mathrm{EH}$ \\
\hline $50-32-8$ & Benzo(a)pyrene & $<0.50$ & $\mathrm{NA}$ & $\mathrm{NA}$ & $\overline{\mathrm{NA}}$ & NA & $\mathrm{EH}$ \\
\hline $193-39-5$ & Indeno(1,2,3-cd)pyrene & $<0.50$ & $\mathrm{NA}$ & NA & $\mathrm{NA}$ & NA & $\mathrm{EH}$ \\
\hline $53-70-3$ & Dibenzo(a,h)anthracene & $<0.50$ & NA & $\mathrm{NA}$ & NA & NA & EH \\
\hline $191-24-2$ & Benzo(g,h,i)perylene & $<0.50$ & $\mathrm{NA}$ & $\mathrm{NA}$ & $\mathrm{NA}$ & $\mathrm{NA}$ & $\mathrm{EH}$ \\
\hline
\end{tabular}

$\mathrm{ND}=$ Compound was analyzed for, but not detected above the laboratory reporting limit.

$\mathrm{NA}=$ Not Applicable

$E H=$ Sample extracted outside of extraction hold time, sample results should be evaluated. 


\section{RESULTS OF ANALYSIS \\ PAGE 1 OF 1}

\section{Client : Field Portable Analytical Inc.}

Client Sample ID : DRAO \#3

PAI Sample ID : P2102120-003

PAI Project ID : P2102120

Test Code : Modified EPA TO-13A

Instrument : HP58901I/MSD

Analyst : Nelyn Quitoviera

Matrix : PUF/XAD-2
Date Sampled : 8/31/01

Date Received : 9/14/01

Date Extracted : 9/24/01

Date Analyzed : 9/27/01

Final Volume: $\quad 1.0 \mathrm{ml}$

Volume Analyzed : $\quad$ NA Liter(s)

D.F. $=1.00$

D.F. $=2.00$

\begin{tabular}{|c|c|c|c|c|c|c|c|}
\hline CAS\# & COMPOUND & $\begin{array}{l}\text { RESULT } \\
\mu \mathrm{g} / \text { Sample }\end{array}$ & $\begin{array}{c}\text { RESULT } \\
\mu \mathrm{g} / \mathrm{m} 3\end{array}$ & $\begin{array}{c}\text { REPORTING } \\
\text { LIMIT } \\
\mu \mathrm{g} / \mathrm{m} 3\end{array}$ & $\begin{array}{c}\text { RESULT } \\
\text { ppbV }\end{array}$ & $\begin{array}{l}\text { REPORTING } \\
\text { LIMIT } \\
\text { ppbV }\end{array}$ & $\begin{array}{c}\text { Data } \\
\text { Qualifier }\end{array}$ \\
\hline $91-20-3$ & Naphthalene & 10 & $\overline{\mathrm{NA}}$ & $\overline{\mathrm{NA}}$ & NA & $\overline{N A}$ & $\overline{\mathrm{EH}}$ \\
\hline $208-96-8$ & Acenaphthalene & $<0.50$ & NA & $\mathrm{NA}$ & $\mathrm{NA}$ & $\mathrm{NA}$ & $\mathrm{EH}$ \\
\hline $83-32-9$ & Acenaphthene & $<0.50$ & $\mathrm{NA}$ & $\mathrm{NA}$ & $\mathrm{NA}$ & NA & $\mathrm{EH}$ \\
\hline $86-73-7$ & Fluorene & $<0.50$ & $\mathrm{NA}$ & $\overline{\mathrm{NA}}$ & NA & $\mathrm{NA}$ & $\mathrm{EH}$ \\
\hline $85-01-8$ & Phenanthrene & $<0.50$ & NA & $\overline{\mathrm{NA}}$ & NA & $\mathrm{NA}$ & EH \\
\hline $120-12-7$ & Anthracene & $<0.50$ & $\mathrm{NA}$ & NA & NA & NA & EH \\
\hline $206-44-0$ & Fluoranthene & $<0.50$ & NA & $\overline{\mathrm{NA}}$ & $\overline{\mathrm{NA}}$ & NA & $\mathrm{EH}$ \\
\hline $129-00-0$ & Pyrene & $<0.50$ & $\mathrm{NA}$ & NA & $\mathrm{NA}$ & $\mathrm{NA}$ & $\mathrm{EH}$ \\
\hline $56-55-3$ & Benzo(a)anthracene & $<0.50$ & $\mathrm{NA}$ & NA & $\mathrm{NA}$ & NA & $\mathrm{EH}$ \\
\hline $218-01-9$ & Chrysene & $<0.50$ & NA & $\mathrm{NA}$ & NA & $\mathrm{NA}$ & $\mathrm{EH}$ \\
\hline $205-99-2$ & Benzo(b)fluoranthene & $<0.50$ & NA & NA & NA & $\mathrm{NA}$ & $\mathrm{EH}$ \\
\hline $207-08-9$ & Benzo(k)fluoranthene & $<0.50$ & NA & $\mathrm{NA}$ & $\mathrm{NA}$ & NA & $\mathrm{EH}$ \\
\hline $50-32-8$ & Benzo(a)pyrene & $<0.50$ & $\mathrm{NA}$ & $\overline{\mathrm{NA}}$ & $\overline{\mathrm{NA}}$ & $\mathrm{NA}$ & $\mathrm{EH}$ \\
\hline $193-39-5$ & Indeno( $1,2,3$-cd)pyrene & $<0.50$ & NA & NA & $\mathrm{NA}$ & NA & $\overline{\mathrm{EH}}$ \\
\hline $53-70-3$ & Dibenzo(a,h)anthracene & $<0.50$ & $\mathrm{NA}$ & NA & NA & NA & $\mathrm{EH}$ \\
\hline $191-24-2$ & Benzo(g,h,i)perylene & $<0.50$ & $\mathrm{NA}$ & NA & NA & $\mathrm{NA}$ & $\mathrm{EH}$ \\
\hline
\end{tabular}

$\mathrm{ND}=$ Compound was analyzed for, but not detected above the laboratory reporting limit.

NA $=$ Not Applicable

$E H=$ Sample extracted outside of extraction hold time, sample results should be evaluated. 


\title{
Performance Analytical Inc.
}

Air Quality Laboratory

A Division of Columbia Analytical Services, Inc.

An Employee Orned Company

\section{RESULTS OF ANALYSIS \\ PAGE 1 OF 1}

\section{Client : Field Portable Analytical Inc.}

\section{Client Sample ID : Method Blank}

PAI Sample ID : P010924-MB

\author{
AI Project ID : P2102120 \\ Test Code : Modified EPA TO-13A \\ Instrument : HP5890II/MSD \\ Analyst : Nelyn Quitoviera \\ Matrix : PUF/XAD-2
}

$\begin{array}{rc}\text { Date Sampled : } & \text { NA } \\ \text { Date Received : } & \text { NA } \\ \text { Date Extracted : } & 9 / 24 / 01 \\ \text { Date Analyzed : } & 9 / 27 / 01 \\ \text { Final Volume: } & 1.0 \mathrm{ml} \\ \text { Volume Analyzed : } & \text { NA Liter(s) }\end{array}$

D.F. $=1.00$

\begin{tabular}{|c|c|c|c|c|c|c|}
\hline CAS \# & COMPOUND & $\begin{array}{l}\text { RESULT } \\
\mu \mathrm{g} / \text { Sample }\end{array}$ & $\begin{array}{c}\text { RESULT } \\
\mu \mathrm{g} / \mathrm{m} 3 \\
\end{array}$ & $\begin{array}{c}\text { REPORTING } \\
\text { LIMIT } \\
\mu \mathrm{g} / \mathrm{m} 3 \\
\end{array}$ & $\begin{array}{c}\text { RESULT } \\
\mathrm{ppbV}\end{array}$ & \begin{tabular}{|c} 
REPORTING \\
LIMIT \\
ppbV \\
\end{tabular} \\
\hline $91-20-3$ & Naphthalene & $<1.0$ & NA & $\mathrm{NA}$ & $\overline{\mathrm{NA}}$ & NA \\
\hline $208-96-8$ & Acenaphthalene & $<0.50$ & NA & $\mathrm{NA}$ & $\mathrm{NA}$ & NA \\
\hline $83-32-9$ & Acenaphthene & $<0.50$ & NA & NA & NA & $\mathrm{NA}$ \\
\hline $86-73-7$ & Fluorene & $<0.50$ & NA & $\mathrm{NA}$ & NA & NA \\
\hline $85-01-8$ & Phenanthrene & $<0.50$ & NA & NA & NA & $\mathrm{NA}$ \\
\hline $120-12-7$ & Anthracene & $<0.50$ & NA & NA & $\mathrm{NA}$ & NA \\
\hline $206-44-0$ & Fluoranthene & $<0.50$ & NA & NA & NA & NA \\
\hline $129-00-0$ & Pyrene & $<0.50$ & $\mathrm{NA}$ & NA & $\mathrm{NA}$ & NA \\
\hline $56-55-3$ & Benzo(a)anthracene & $<0.50$ & $\mathrm{NA}$ & NA & $\mathrm{NA}$ & $\mathrm{NA}$ \\
\hline $218-01-9$ & Chrysene & $<0.50$ & NA & NA & $\mathrm{NA}$ & $\mathrm{NA}$ \\
\hline $205-99-2$ & Benzo(b)fluoranthene & $<0.50$ & NA & $\mathrm{NA}$ & $\mathrm{NA}$ & NA \\
\hline $207-08-9$ & Benzo(k)fluoranthene & $<0.50$ & $\mathrm{NA}$ & NA & $\mathrm{NA}$ & $\mathrm{NA}$ \\
\hline $50-32-8$ & Benzo(a)pyrene & $<0.50$ & $\mathrm{NA}$ & $\mathrm{NA}$ & $\mathrm{NA}$ & NA \\
\hline $193-39-5$ & Indeno(1,2,3-cd)pyrene & $<0.50$ & NA & $\mathrm{NA}$ & $\mathrm{NA}$ & NA \\
\hline $53-70-3$ & Dibenzo(a,h)anthracene & $<0.50$ & NA & NA & NA & NA \\
\hline $191-24-2$ & Benzo(g,h,i)perylene & $<0.50$ & NA & NA & NA & $\mathrm{NA}$ \\
\hline
\end{tabular}

$\mathrm{ND}=$ Compound was analyzed for, but not detected above the laboratory reporting limit.

$\mathrm{NA}=$ Not Applicable

Ke Date: $10 /$ sla 


\section{Appendix B}

\section{NDEP Comment Responses}




\section{NEVADA ENVIRONMENTAL RESTORATION PROJECT}

\section{DOCUMENT REVIEW SHEET}

\begin{tabular}{|c|c|c|c|c|c|}
\hline \multicolumn{4}{|c|}{$\begin{array}{l}\text { 1. Document Title/Number: Draft Post-Closure Monitoring Report for Corrective Action Unit 329: } \\
\text { Area } 22 \text { Desert Rock Airstrip Fuel Spill, Nevada Test Site, Nevada }\end{array}$} & \multicolumn{2}{|l|}{ 2. Document Date: February 2002} \\
\hline \multicolumn{4}{|c|}{ 3. Revision Number: 0} & \multicolumn{2}{|c|}{ 4. Originator/Organization: IT Corporation } \\
\hline \multicolumn{6}{|c|}{ 7. Review Criteria: Full } \\
\hline \multicolumn{4}{|c|}{ 8. Reviewer/Organization/Phone No.: John A. Wong, NDEP, 486-2866 } & \multicolumn{2}{|l|}{ 9. Reviewer's Signature: } \\
\hline 1) General & & Insert a table of contents at the beginning of the report. & \multicolumn{2}{|c|}{ Table of contents was added. } & Yes \\
\hline $\begin{array}{l}\text { 2) Page ES-1, } \\
2^{\text {nd }} \text { Sentence }\end{array}$ & & $\begin{array}{l}\text { Indicate the duration for which monitoring is planned/required } \\
\text { (i.e., annually for } 5 \text { years). }\end{array}$ & \multicolumn{2}{|c|}{ The monitoring duration was added. } & Yes \\
\hline 3) General & & $\begin{array}{l}\text { A section of the report should contain a discussion on how the } \\
\text { determination of biodegradation is going to be made with respect to } \\
\text { the data generated and reported for each event (i.e., what the } \\
\text { objectives for the data are, and what types of results might be } \\
\text { anticipated). NDEP understands that this set of data, being the first } \\
\text { set of data generated since the collection of baseline data in } \\
\text { August } 2000 \text {, does not necessarily provide definitive information on } \\
\text { the rates of biodegradation. However, an evaluation of } \\
\text { biodegradation rates will eventually be made using the data, so } \\
\text { data/results should be discussed within this context. Additionally, } \\
\text { this section should include NNSA/NV's current evaluation of } \\
\text { whether or not the residual soil contamination is undergoing natural } \\
\text { attenuation, and whether or not contaminant migration is occurring. } \\
\text { If future sampling events yield inconclusive data, NNSA/NV will } \\
\text { need to explore and present alternative remediation strategies } \\
\text { before the site can be closed. }\end{array}$ & \multicolumn{2}{|c|}{$\begin{array}{l}\text { A discussion was added on how the determination of bio- } \\
\text { degradation will be made. } \\
\text { Statements saying that natural attenuation may be occurring } \\
\text { and that contaminant migration is not occurring were added. }\end{array}$} & Yes \\
\hline $\begin{array}{l}\text { 4) Section 3.0, } \\
\text { Conclusions }\end{array}$ & & $\begin{array}{l}\text { An interpretation of the results is necessary, even if this means } \\
\text { stating that the data do not support effective biodegradation at this } \\
\text { point. }\end{array}$ & \multicolumn{2}{|c|}{ An Interpretation was added. } & Yes \\
\hline
\end{tabular}

${ }^{a}$ Comment Types: $M=$ Mandatory, $S=$ Suggested

Return Document Review Sheets to NNSA/NV Environmental Restoration Division, Attn: QAC, M/S 505 


\section{Distribution}

*Provide copy in initial distribution of Rev. 0 and subsequent revisions, if applicable. Copies of the NDEP-approved document will be distributed to others.

\section{$\underline{\text { Copies }}$}

Paul J. Liebendorfer

1 (Controlled)*

State of Nevada

Bureau of Federal Facilities

Division of Environmental Protection

333 W. Nye Lane, Room 138

Carson City, NV 89706-0851

Donald R. Elle, Las Vegas Office

1 (Controlled)*

State of Nevada

Bureau of Federal Facilities

Division of Environmental Protection

1771 E. Flamingo Road, Suite 121-A

Las Vegas, NV 89119

Sabrina Lawrence

Environmental Restoration Division

1 (Controlled)*

U.S. Department of Energy

National Nuclear Security Administration

Nevada Operations Office

P.O. Box 98518, M/S 505

Las Vegas, NV 89193-8518

Janet Appenzeller-Wing

1 (Uncontrolled)*

Environmental Restoration Division

U.S. Department of Energy

National Nuclear Security Administration

Nevada Operations Office

P.O. Box 98518, M/S 505

Las Vegas, NV 89193-8518

Sabine Curtis

1 (Uncontrolled)*

Environmental Restoration Division

U.S. Department of Energy

National Nuclear Security Administration

Nevada Operations Office

P.O. Box 98518, M/S 505

Las Vegas, NV 89193-8518 


\section{$\underline{\text { Copies }}$}

IT Corporation Central Files

1 (Uncontrolled)*

P.O. Box 93838

Las Vegas, NV 89193

Jeffrey Johnson

1 (Uncontrolled)*

IT Corporation

P.O. Box 93838

Las Vegas, NV 89193

Manager, Northern Nevada FFACO

1 (Uncontrolled)

Public Reading Facility

c/o Nevada State Library \& Archives

100 North Stewart Street

Carson City, NV 89701-4285

Technical Information Resource Center

U.S. Department of Energy

National Nuclear Security Administration

Nevada Operations Office

P.O. Box $98518, \mathrm{M} / \mathrm{S} 505$

Las Vegas, NV 89193-8518

U.S. Department of Energy

Office of Scientific and Technical Information

1 (Uncontrolled, electronic copy)

P.O. Box 62

Oak Ridge, TN 37831

Manager, Southern Nevada FFACO

1 (Controlled)

Public Reading Facility

1 (Uncontrolled)

P.O. Box 98521, M/S NLV040

Las Vegas, NV 89193-8521

FFACO Support Office

1 (Controlled)

IT Corporation

P.O. Box 93838

Las Vegas, NV 89193

1 (Uncontrolled)

Earl Dixon

Community Advisory Board

1 (Uncontrolled)

UNLV, Harry Reid Center for Environmental Studies 4505 S. Maryland Pkwy

Las Vegas, NV 89154 
CAU 329 Post-Closure Report

Distribution List

Revision: 0

Date: 06/07/2002

Page 3 of 3

\section{$\underline{\text { Copies }}$}

Phillip Claire, CAB Chairman

1 (Uncontrolled)

6252 Santander Avenue

Las Vegas, NV 89103 